Florida International University FIU Digital Commons

6-16-2016

\title{
Economic Aspects of Climate Change Adaptation and Natural Hazard Risk Mitigation
}

Sisi Meng

smeng003@fiu.edu

DOI: $10.25148 /$ etd.FIDC000696

Follow this and additional works at: https://digitalcommons.fiu.edu/etd

Part of the Economic Policy Commons, and the Economics Commons

\section{Recommended Citation}

Meng, Sisi, "Economic Aspects of Climate Change Adaptation and Natural Hazard Risk Mitigation" (2016). FIU Electronic Theses and Dissertations. 2630.

https://digitalcommons.fiu.edu/etd/2630

This work is brought to you for free and open access by the University Graduate School at FIU Digital Commons. It has been accepted for inclusion in FIU Electronic Theses and Dissertations by an authorized administrator of FIU Digital Commons. For more information, please contact dcc@fiu.edu. 


\section{FLORIDA INTERNATIONAL UNIVERSITY \\ Miami, Florida}

\section{ECONOMIC ASPECTS OF CLIMATE CHANGE ADAPTATION AND NATURAL HAZARD RISK MITIGATION}

A dissertation submitted in partial fulfillment of the requirements for the degree of DOCTOR OF PHILOSOPHY

in ECONOMICS

by

Sisi Meng 
To: Dean John F. Stack, Jr.

Steven J. Green School of International and Public Affairs

This dissertation, written by Sisi Meng, and entitled Economic Aspects of Climate Change Adaptation and Natural Hazard Risk Mitigation, having been approved in respect to style and intellectual content, is referred to you for judgment.

We have read this dissertation and recommend that it be approved.

$\begin{array}{r}\hline \text { Mihaela Pintea } \\ \hline \text { Sheng Guo } \\ \hline \text { Pallab Mozumder, Major Professor }\end{array}$

Date of Defense: June 16, 2016

The dissertation of Sisi Meng is approved.

Dean John F. Stack, Jr.

Steven J. Green School of International and Public Affairs

Andrés G. Gil

Vice President for Research and Economic Development and Dean of the University Graduate School

Florida International University, 2016 
(C) Copyright 2016 by Sisi Meng

All rights reserved. 


\section{DEDICATION}

This dissertation is dedicated to my beloved parents. Thank you for all of the patience, support, encouragement, and love. 


\section{ACKNOWLEDGMENTS}

I wish to express my deep appreciation and gratitude to my major advisor, Dr. Pallab Mozumder, for the mentorship and guidance he provided to me in my academic pursuit. I am truly grateful to have had the opportunity to work with him and learn from him. I would also like to thank all the members of my committee, Dr. Mihaela Pintea, Dr. Mahadev Bhat, and Dr. Sheng Guo, for their insightful comments and supports. Special thanks goes to Dr. Maria Willumsen. I am indebted to her for her continuous encouragement, support, and love.

In a similar vein, I would like to thank Dr. John Boyd, Dr. Cem Karayalcin, Dr. Hakan Yilmazkuday, Dr. Jesse Bull, and all other faculty members for their dedication during my Ph.D. studies at Florida International University. I am also grateful to the following former and current staffs at the Department of Economics for their generous supports during my graduate studies - Mariela Delgado, Mayte Rodriguez, Maria Cossio, and Lorette Garcia.

I would also like to acknowledge the supports from the National Science Foundation (Award \#0838683, \#1204762), the Florida Division of Emergency Management (DEM), and the International Hurricane Research Center at FIU. I am also thankful to all those respondents who participated in our survey and GFK (formerly Knowledge Networks) staff members who implemented the survey.

Finally, I would like to thank FIU's University Graduate School for the support provided through the Dissertation Year Fellowship from Spring 2016 to Summer 2016. 


\section{ABSTRACT OF THE DISSERTATION \\ ECONOMIC ASPECTS OF CLIMATE CHANGE ADAPTATION AND \\ NATURAL HAZARD RISK MITIGATION}

by

Sisi Meng

Florida International University, 2016

Miami, Florida

Professor Pallab Mozumder, Major Professor

According to Munich Re (2013), economic losses related to natural disasters have increased from an average of $\$ 50$ billion in the 1980 s to $\$ 200$ billion over the last decade. The cost of natural disasters is accumulating rapidly and some claim that climate change is responsible. Others believe that human behaviors like population growth or land use should be blamed for these rising costs. The process of climate change has already taken place, and it is expected to continue to impact the future. As a result, people are more vulnerable today. Therefore, understanding the economic aspects of climate change and natural hazard risks should be considered as a major issue and addressed in greater detail. This dissertation aimed to explore household preferences of climate change adaptation and the economic impacts of natural hazards at both micro- and macro- levels.

The dissertation consisted of three related empirical studies based on the two main changes that will occur with climate change predicted by scientific climate models: stronger hurricanes and rising sea levels. The first chapter examined the impact of a recent hurricane on household activities. The objective was to find out whether a more intensified hurricane caused greater damages, and whether such damages had a long-lasting impact on household recovery. If the impact of natural hazards is worse than before, people should avoid putting themselves in harm's way. 
However, evidence indicates that the population in coastal cities is still growing fast, as people tend to reside near the beaches and attractive landscapes. Concerns are thus prompted by the possible lack of perceptions for future risks caused by natural hazards. Therefore, the second chapter focused on household perceptions and preferences for adapting to sea level rise in Florida. Lastly, although a disaster strikes rich or poor nations indifferently, some small island nations are among the most vulnerable. In the third chapter, the macroeconomic implications of natural hazards in Central America and the Caribbean were investigated. A careful examination of the economic factors that can lead to smaller losses and higher abilities to cope with disasters is crucial in such countries. 


\section{TABLE OF CONTENTS}

CHAPTER

PAGE

1. HURRICANE SANDY: DAMAGES, DISRUPTIONS AND PATHWAYS TO

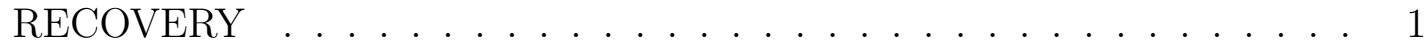

1.1 Introduction and Background . . . . . . . . . . . . . . 1

1.2 The Determinants of Household Recovery _. . . . . . . . . . . . . 3

1.3 Dynamic Framework of Household Disaster Resilience . . . . . . . . . . . 8

1.3.1 Utility Disruption and Resilience . . . . . . . . . . . . . . . . . 8

1.3.2 A Household Decision Framework . . . . . . . . . . . . . . . . . . . 10

1.3.3 Dynamic Framework of Resilience and Preparedness . . . . . . . . . . 11

1.4 Data and Sample Characteristics . . . . . . . . . . . . . . . . 15

1.5 Econometric Specification . . . . . . . . . . . . . . . . . . . 19

1.6 Estimation Results . . . . . . . . . . . . . . . . . . . . . . . . . . 22

1.7 Conclusion . . . . . . . . . . . . . . . . . . . . . . . . . . . . 27

References . . . . . . . . . . . . . . . . . . . . . . 29

Tables . . . . . . . . . . . . . . . . . . . . . . . . 35

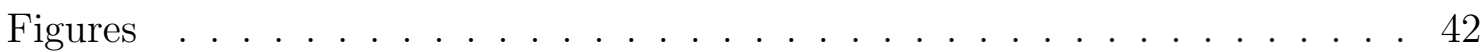

2. SPATIAL HETEROGENEITY AND HOUSEHOLD PREFERENCES FOR SEA LEVEL RISE ADAPTATION PLAN IN FLORIDA . . . . . . . . 52

2.1 Introduction . . . . . . . . . . . . . . . . . . . 52

2.2 Spatial Heterogeneity and Preferences for Adaptation . . . . . . . . . . 55

2.2.1 Previous Research on Climate Change Adaptation . . . . . . . . . . . 55

2.2 .2 Distance and Spatial Heterogeneity . . . . . . . . . . . . . . . . 57

2.2.3 Preferences in the Short and Long Term . . . . . . . . . . . . . . 58

2.2.4 Preferences among Yearlong and Seasonal Residents . . . . . . . . . 59

2.3 Survey and Choice Experiment Design . . . . . . . . . . . . . . . 60

2.4 Sample Characteristics . . . . . . . . . . . . . . . . . . . . . . 63

2.4 .1 Survey Data . . . . . . . . . . . . . . . . . . 63

2.4 .2 GIS Data . . . . . . . . . . . . . . . . . . . . . . 65

2.5 The Choice Experiment . . . . . . . . . . . . . . . . . . . . . . . 67

2.6 Choice Experiment Results . . . . . . . . . . . . . . . . . . . . . . . . 71

2.6.1 Results from the Short-term Choice . . . . . . . . . . . . . . . . . 71

2.6.2 Results from the Long-term Choice . . . . . . . . . . . . . . . . . . 75

2.7 WTP and Economic Values for SLR Adaptation Plan . . . . . . . . . . 77

2.8 Conclusion . . . . . . . . . . . . . . . . . . . . . . . . . . . . . . . . . 79

References . . . . . . . . . . . . . . . . . . . . . . . . . . . . 82

Tables . . . . . . . . . . . . . . . . . . . . . . 87

Figures $\ldots \ldots \ldots \ldots \ldots$ 
3. INSTITUTIONS AND NATURAL DISASTERS: EVIDENCE FROM AGRICULTURAL TRADE IN CENTRAL AMERICA AND THE CARIBBEAN 103

3.1 Introduction . . . . . . . . . . . . . . . . . . . . . . . . . 103

3.2 Literature Review . . . . . . . . . . . . . . . . . . . . . . . 106

3.2 .1 The Impact of Natural Disasters . . . . . . . . . . . . . . . . . 106

3.2 .2 The Role of Institution . . . . . . . . . . . . . . . . . . . . . . 109

3.3 Data . . . . . . . . . . . . . . . . . . . . . . . . 111

3.3.1 Agricultural Trade Data . . . . . . . . . . . . . . . . . . . . . 111

3.3.2 Natural Disasters Data . . . . . . . . . . . . . . . . . . . . . . . 111

3.3.3 Institutional Quality Data . . . . . . . . . . . . . . . . 115

3.4 Empirical Analysis . . . . . . . . . . . . . . . . . . . . . . . 116

3.4 .1 Model Specification . . . . . . . . . . . . . . . . . . . . . 116

3.4.2 Choice of Institution Indicators . . . . . . . . . . . . . . . . . . . 119

3.4.3 Regression Results . . . . . . . . . . . . . . . . . . . . . . . . 120

3.5 The Impact of Storms and Hurricanes . . . . . . . . . . . . . 126

3.5.1 The Impact of Storms . . . . . . . . . . . . . . . . . 126

3.5.2 The Impact of Hurricanes . . . . . . . . . . . . . . . . . . . . 128

3.6 Conclusion . . . . . . . . . . . . . . . . . . . . . . . . . . . 130

References . . . . . . . . . . . . . . . . . . . . . . 133

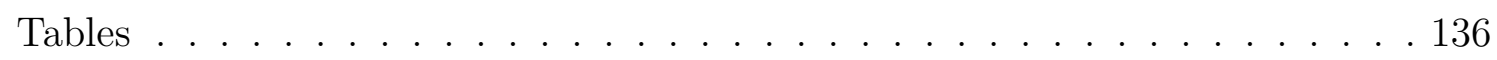

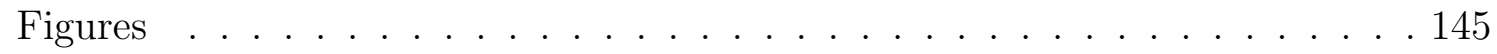

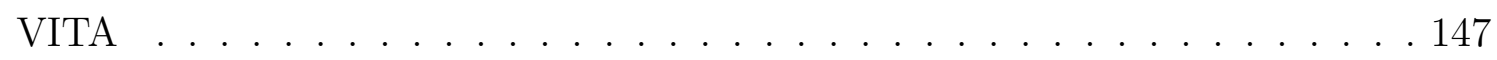




\section{LIST OF TABLES}

TABLE

PAGE

1.1 Description of Variables . . . . . . . . . . . . . . . . 35

1.2 Descriptive Statistics of Variables in the Sample . . . . . . . . . . 36

1.3 Estimation Results for Household Damage and Recovery . . . . . . . . . 37

1.4 Marginal Effects for Household Damage and Recovery . . . . . . . . . . 39

1.5 Predicted Probabilities on Recovery by Utility Disruption . . . . . . . . 40

1.6 Predicted Probabilities on Recovery by Irene and Education . . . . . . . 41

2.1 Attribute Levels Used in the Survey . . . . . . . . . . . . . . . . . 87

2.2 Descriptive Statistics of Respondent Characteristics . . . . . . . . . . . 88

2.3 Descriptive Statistics of Other Respondent Information . . . . . . . . . 89

2.4 Results of Multinomial Logit Model (Short Term) . . . . . . . . . . . . 90

2.5 Results Between Yearlong and Seasonal Residents (Short Term) . . . . . 91

2.6 Results of Multinomial Logit Model (Long Term) . . . . . . . . . . . . . 92

2.7 Results Between Yearlong and Seasonal Residents (Long Term) . . . . . 93

2.8 MWTP for Attributes in the Short and Long Term . . . . . . . . . . . . 94

3.1 List of Countries and the Number of Events . . . . . . . . . . . . 136

3.2 Summary of Used Variables . . . . . . . . . . . . . . . . . 137

3.3 Correlation between Six Institutional Indicators . . . . . . . . . . . . . 138

3.4 Explanatory Power of Six Institutional Indicators . . . . . . . . . . . . . 139

3.5 The Impact of Disasters on Agricultural Imports . . . . . . . . . . . . . 140

3.6 The Impact of Disasters on Agricultural Exports . . . . . . . . . . . . . 141

3.7 The Role of the Institution . . . . . . . . . . . . . . . . . . . . . 142

3.8 Regression Results on the Impact of Storms . . . . . . . . . . . . . . . 143

3.9 Regression Results on the Impact of Hurricanes . . . . . . . . . . . . . . 144 


\section{LIST OF FIGURES}

FIGURE

PAGE

1.1 The Disaster Resilience Triangle . . . . . . . . . . . . . . . . . . . . . 42

1.2 The Decision Framework . . . . . . . . . . . . . . . . . . . . . 43

1.3 Dynamic Framework of Household Resilience . . . . . . . . . . . . . . . 44

1.4 Survey Questions on Damage and Recovery . . . . . . . . . . . . . . 45

1.5 Location of Surveyed Respondents by State . . . . . . . . . . . . . 46

1.6 Statistics on Utility Disruptions _ . . . . . . . . . . . . 47

1.7 Predicted Probabilities on Damage by Wind Speed . . . . . . . . . . . 48

1.8 Predicted Probabilities on Recovery by Utility Disruption . . . . . . . . 49

1.9 The Role of Installing Window Protection on Recovery . . . . . . . . . . 50

1.10 The Role of Owning a Generator on Recovery . . . . . . . . . . . . . 51

2.1 Impact of SLR on Areas of Florida from Year 2030 to 2010 . . . . . . . 95

2.2 Estimated Projection in Florida by USACE . . . . . . . . . . . . 96

2.3 Differences in Risk Perception among Yearlong and Seasonal Residents . 97

2.4 Differences in Concerns among Yearlong and Seasonal Residents . . . . . 98

2.5 Elevation Map of Florida and Locations of Surveyed Respondents . . . . 99

2.6 Areas of Inundation in Florida and the Affected Respondents . . . . . 100

3.1 Natural Disaster Subgroup Definition and Classification . . . . . . . 145

3.2 Number of Disasters and Storms by Country . . . . . . . . . . . 146 


\section{CHAPTER 1}

\section{HURRICANE SANDY: DAMAGES, DISRUPTIONS AND PATHWAYS TO RECOVERY}

\subsection{Introduction and Background}

Critical infrastructure and public utility systems that provide goods and services are often severely damaged by natural disasters, such as hurricanes. On the 29th of October 2012, Hurricane Sandy made landfall in the United States as a Category 2 hurricane, striking near Atlantic City, New Jersey. The storm caused widespread and long-lasting disruptions to infrastructure systems and utility services, especially in the New York and New Jersey areas. More than 8.2 million people across the east coast immediately lost power (Breed \& Hays, 2012) and more than 1.3 million remained without electricity for up to a week (Sledge, 2012). Depleted fuel supplies led to long lines at the gas stations which stretched on for up to a mile or more (Smith \& Maglio, 2012). The transportation systems also stopped immediately in preparation for the storm and did not resume services until several days later. Over 15,000 scheduled commercial flights were canceled both in the U.S. and around the world (Breed \& Hays, 2012). High winds, heavy storms and flooding brought massive interruptions to phone services, wireless connections and other communication networks along Sandy's path.

The impact of utility disruptions can halt ordinary economic activities from minor inconveniences (such as power outages of short duration and delays to economic transactions), to more serious disruptions from extended loss of utilities and public services for days and weeks, and the long-term shut-down of bridges, roads and other transportation networks. However, the impacts, especially at the household level, arising from these inconveniences are generally overlooked in the natural hazard lit- 
erature (Vogel, 2002). Hurricane Sandy's impact on the economy and infrastructure affected a total of 24 U.S. states, providing an avenue to observe the role of utility disruption on households in the affected area.

Bruneau et al. (2003) introduced a framework of disaster resilience to measure the ability of physical infrastructure to perform during and after a disaster. They defined community resilience as the ability of social units (e.g., organizations, communities) to mitigate hazards, contain the effects of disasters when they occur, and carry out recovery activities in ways that minimize social disruption and mitigate the potential impacts of future disasters. The definition can be further divided into three aspects: reduced failure probabilities, reduced consequences, and reduced time to recovery. In this study we concentrate on the third aspect of community resilience: more rapid recovery.

Moreover, we extend the framework of community resilience in two ways: First, we shift the focus of the research from the community level to a more decentralized household level. We define household disaster resilience as the ability of households to recover from the adverse shocks of a disaster. Study at a more disaggregated micro level is necessary, because the economic research on natural disasters and their consequences including the household-level recovery process is still limited (Takashi, 2012). Second, we incorporate the role of preparedness activities and socioeconomic characteristics into the framework. The concept of disaster resilience goes beyond physical infrastructure, and proactive preparedness activities are observed to promote higher resilience and inhibit the impacts of disastrous events (Resurreccion \& Santos, 2013). Socio-economic characteristics have also been shown to determine households' ability to cope with natural disasters (Mozumde et al., 2009).

It is also worth mentioning that household recovery is not just about the restoration of household well-being to its pre-existing level. It is also about assessing the 
vulnerabilities and developing mitigation approaches that increase future resiliency (Murphy, in press). The recovery period can offer opportunities to facilitate economic, social and physical development, and to alter physical development patterns to reduce future hazard vulnerability (Berke, Kartez, \& Wenger, 1993). Past literature concentrated more on the immediate aftermath following the event, with little focus on the long-term recovery phase (Mddonnell et al., 1995). Against this backdrop, this study aims to investigate factors contributing to longer-term recovery as they will impact future levels of households' vulnerability and resiliency.

The main goal of this study is to estimate the effects of utility disruptions on household-level recovery, based on an extended framework of household disaster resilience. We also discuss the role of preparedness activities and socio-economic characteristics in the recovery process. More specifically, we attempt to answer three questions: How do utility disruptions affect households' recovery? To what extent do these disruptions affect households? What types of households are more resilient (that is, faster in recovering) from Hurricane Sandy? To address these questions, we have conducted a household survey eight months after Hurricane Sandy. Respondents were first asked to state their monetary damages caused by Sandy and those who reported a positive amount of damage were asked to rate their recovery levels at the second stage. We have taken into account the sample selection bias, although we are mainly concerned with those seriously affected households.

\subsection{The Determinants of Household Recovery}

Hurricanes adversely impact household activities in a number of ways. The immediate consequences of the disaster impact include loss of life, injury and physical damages. Indirect impacts are losses in well-being resulted from changes in the level 
of activity, for example, increases in unemployment or losses in income. In the aftermath of a hurricane, the community is forced to address a number of important issues. Once critical emergency services and immediate life safety issues have been addressed, the community can turn its attention to the post-disaster recovery efforts: return of evacuees, reconstruction, financial assistance, and restoration of vital infrastructures (Vogel, 2001). The challenge to researchers is then to identify the drivers that allow a region to reduce the impact of disruption and promote recovery (Ewing, Kruse, \& Sutter, 2007).

The previous literature has emphasized community dependence on the restoration of utility services in the recovery process. Bruneau et al. (2003) indicated that improving the resilience of critical lifelines, such as water, power, and critical facilities, is instrumental for overall community resilience. These organizations form the "backbone" for community functioning; they enable communities to cope and respond disaster strikes. Liu et al. (2005) highlighted that hurricane-related damages to the electric power system can cause significant economic losses, business interruptions, and costly restoration efforts. Power outage may also result in interruption of security systems, financial transactions, communication, health care, water distribution, traffic signaling, and other lifeline systems that depend on electricity. It is highly possible that communities suffer from cascading effects of service disruptions, because failures in one system can lead to disruptions in others, exacerbating response and recovery efforts (Chang et al., 2014). Therefore, restoration following a hazardous event is critical to the rapid recovery of the affected communities (Han, Guikema, \& Quiring, 2009; Reed, 2008).

A number of studies have tried to quantitatively estimate the impact of utility disruption. Chang, Svekla, and Shinozuka (2002) developed an integrated engineeringeconomic loss estimation model to explore how damages caused by an earthquake 
translate into economic losses. Bruneau et al. (2003) presented a framework for defining seismic resilience and specifying quantitative measures of resilience. Three complementary measures of resilience are keys to their framework: reduced failure probabilities, reduced consequences from failures, and reduced time to recovery. Alternative approaches to hazard loss estimation included input-output (I-O) models and computable general equilibrium (CGE) models. For example, Rose and Liao (2005) studied the impacts of water supply disruptions due to an earthquake in the Portland metropolitan area, by using CGE analysis. Several other studies focused on estimating the effects of utility disruptions on business discontinuity and resilience (Nigg, 1995; Tierney \& Nigg, 1995). Their research is important because recovery may depend on infrastructure and business resilience (Rose \& Lim, 2002).

However, very few theoretical and empirical works studying the role of utility disruption have been developed at the household level. A few researchers used contingent valuation techniques to elicit households' willingness to pay to avoid power outage, or to increase water supply reliability in general (Carlsson, Martinsson, \& Akay, 2011; Griffin \& Mjelde, 2000; Howe et al., 1994). Chang and Miles (2004) described a simulation model of disaster recovery that included not just the whole community, but its interactions between households, businesses and infrastructure systems. Nonetheless, other than Chang and her coauthor's works, no conceptual frameworks of the recovery process at the household level were found in the literature (Marshall \& Schrank, 2014).

On the other hand, previous literature has primarily focused on households' socio-economic characteristics. Prior research has indicated that disasters tend to differentially impact households because of pre-disaster levels of social vulnerability (Cutter, Boruff, \& Shirley, 2003). A common pattern has shown that poor households are particularly vulnerable, since their initial assets are already close to the 
poverty line and their abilities to mobilize resources to cope with negative impacts are limited (Fothergill \& Peek, 2004; Masozera, Bailey, \& Kerchner, 2007; Morris et al., 2002; Sawada, 2007). Age, education and household size are some of the characteristics often examined in the literature. It was suggested that some groups, such as older households or households with a larger family size, are more likely to be displaced by hurricanes (Frankenberg et al., 2011; Morrow, 1999; Peacock, 2003). The elderly or children may have mobility constraints or concerns that increase the burden of care can affect resilience (Cutter et al., 2003). It was also suggested that some groups might be educationally disadvantaged, as lower levels of educational attainments constrain the households' ability to understand necessary information that may expedite recovery. On the contrary, educated people adapt more easily as economic circumstances change, using their assets more efficiently, obtaining better credit arrangements, and exploiting new income opportunities more quickly (Glewwe \& Hall, 1998; Schultz, 1975).

Past hurricane experience may have contributed to the household recovery as well. Research has found that households with higher levels of disaster-related knowledge and experiences are less impacted in the first place because they are more likely to undertake protective actions or adjustments (Faupel \& Styles, 1993; Peacock, Brody, \& Highfield, 2005). In the aftermath of a disaster, knowledge and experience can help households acquire necessary assistances, claim insurances and receive compensation more efficiently. Experienced households are also generally less worried or stressed from the disaster impacts (Hallstrom \& Smith, 2005).

Studies have also shown that preparation activities can be important determinants of the differential impacts of disasters. For households, preparedness involves self-protective actions and obtaining the resources needed for both an effective response and recovery (Tierney, Lindell, \& Perry, 2001). For example, mitigation 
measures targeted to reduce wind related damages (e.g., shutters, hurricane resistant windows and doors) and alternative resources for dealing with emergency situations (e.g., electric generators and hurricane supplies) can be effective (Chatterjee \& Mozumder, 2015).

Insurance coverage is often discussed as an essential element in managing natural disaster risks and promoting recovery from disasters (Botzen, 2013). Households who lost their homes, material possessions and jobs can get their insurance to replace their property losses or lost wages. However, even when insurance is available and households have basic coverage in place, the majority may not have the special perils insurance that is sometimes needed for particular hazards (Murphy, in press). Evidence suggests that the costs of the premiums and lack of knowledge have contributed to the lack of adequate insurance coverage (Mileti, 1999). This further supports the contention that rich and well-aware households are more likely to have adequate coverage, which speeds up recovery. Also, there might be a "moral hazard" issue associated with insurance coverage. Fronstin and Holtmann (1994) pointed out that insurance coverage may reduce the incentive for individuals to protect their property during a hurricane.

In the natural hazard literature, vulnerability and resilience represent two related but different approaches to understand the household response and recovery. Here we reply on the concept of resilience over vulnerability. According to Alinovi, D'Errico, Mane, and Romano (2010), vulnerability tends to measure only the susceptibility of a household to harm and the immediate coping mechanisms adopted. Resilience, however, tries to identify the different responses adopted by a household and capture the "dynamic" components of the adopted strategies. A resilience approach investigates not only how disturbances and changes might influence the households, but also how its functionality in meeting these needs might change. 
Therefore, the concept of resilience fits better for studying the dynamic transformation in the presence of a disaster, from the response to the consequences of shocks, and to the recovery from disaster impacts.

Chiradip and Mozumder (2015) conducted a similar analysis to determine the role of utility disruption on household well-being in the aftermath of Hurricane Wilma. Using household survey data, they concluded that the disruption of public utility services (e.g., water supply, electricity and telephone) and the suspension of local economic activities (e.g., transportation and local businesses) result in significant losses for households' well-being. Along this line we focus on a recent hurricane with more devastating impacts and study the household recovery process, by introducing an extended framework of household disaster resilience. Second, we use a two-stage survey in order to closely examine those households who suffered monetary damages while taking into account the sample selection bias. Third, we include a meteorological variable, wind speed, in order to control for the severity of household exposures to the hurricane event.

\subsection{Dynamic Framework of Household Disaster Resilience}

\subsubsection{Utility Disruption and Resilience}

Bruneau et al. (2003) presented a framework to quantitatively measure the loss of disaster resilience. Zobel (2011) referred this as disaster resilience triangle, which clearly depicted the idea that resilience depends on the extent of damage due to utility disruptions and the time needed to recover. As shown in Figure 1.1, the area of the triangle is measured as:

$$
\text { Loss of Resilience }=\int_{t_{0}}^{t_{1}}[100-Q(t)] d t
$$


where $Q(t)$ is the performance of the infrastructure systems or utility services of a community. If a disaster occurs at time $t=t_{0}$, the performance of infrastructure can degrade from a full service (100\%) to a reduced service level (say 50\%). Over time, the restoration of the infrastructure takes place to bring it to full capacity at time $t_{1}$. The loss of resilience therefore measures the size of the expected degradation in performance over time.

Reed, Kapur, and Christie (2009) further extended this concept to a more specific context of wind-induced damage. They defined the system's performance or level $Q(t)$ by:

$$
Q(t)=Q_{\infty}-\left(Q_{\infty}-Q_{0}\right) e^{-b t}
$$

where $Q_{\infty}$ is the capacity of the fully functioning structural system, $Q_{0}$ is the postdisaster capacity. $b$ is the parameter to measure the speed of the recovery process. Therefore, resilience can be calculated by integrating the area under the $Q(t)$ curve, expressed as:

$$
\text { Resilience }=\frac{\int_{t_{0}}^{t_{1}}[Q(t)] d t}{\left(t_{1}-t_{0}\right)}
$$

where $t_{0}$ and $t_{1}$ are the endpoints of the time interval under consideration.

The concept of resilience has been primarily used within the field of infrastructure engineering. van Bastelaer (2014) recently suggested that it should be considered across a much wider portfolio of development areas, especially in the domain of social sciences. He defined resilience as the capacity of individuals, households and communities to decrease the negative impacts of crises or shocks on their health, economic well-being, and human development. He also pointed out the positive returns of preparation activities by households before disasters.

In this study we apply the framework to quantitatively measure the disaster resilience at the much disaggregated (household) level. We define household disaster resilience as their ability to recover from the adverse shocks of a disaster. We 
also incorporate the benefit of preparedness activities and diverse socio-economic characteristics into the framework. More specifically, we have considered the cost of preparedness, which has not been discussed in the previous literature. Research has shown that socio-economic characteristics are important factors in household preparation decisions. For instance, households with higher socio-economic status (e.g., high-income households) are better prepared and more likely to be insured against disasters (Tierney et al., 2001). If the cost of preparedness is bounded by households' income constraints, it may play an important role on household decisions and responses facing a disaster.

\subsubsection{A Household Decision Framework}

Suppose that a household's well-being $(W)$ is assessed by getting the greatest value possible from the expenditure of goods $Z\left(E_{z}\right)$, derived from the income $(I)$ and the household-related characteristic vector $(H)$. The household's objective is therefore to maximize:

$$
W=W\left(E_{Z}, I, H\right)
$$

subject to an income constraint:

$$
\sum E_{Z} Z \leq I
$$

The initial level of household's well-being can be written as:

$$
W_{0}=W\left(E_{Z 0}, I_{0}, H_{0}\right)
$$

Now, each household in Sandy-affected areas faced a decision to whether engage in preparation actions before the landfall of the hurricane. Once the decision was made and Sandy made landfall, some households could experience higher monetary damages due to the wind-induced utility disruptions. Others may suffer from 
less utility disruptions that cause less damages to the households. The decision framework and the associated outcomes are presented in Figure 1.2.

Starting from the top of the diagram, an effective preparation measure is expected to reduce hurricane-induced damages and alleviate loss of household wellbeing. The net benefit of such decision is measured by the benefit of preparation minus the associated cost $\left(B_{P}-C_{P}\right)$. If the household was prepared and incurred damages, the outcome becomes $B_{P}-C_{P}-C_{D}$, where $C_{D}$ presents the cost of the disruptions. Without preparation, households could yield either an outcome of 0 or an outcome of $-a C_{D}$. Note that the cost of disruptions tends to be worse for unprepared households, captured by a weight $a$, where $a>1$. Figure 1.3 illustrates the above setup to compare the best outcomes with net benefit of preparedness being positive $\left(B_{P}>C_{P}\right)$ or negative $\left(B_{P}<C_{P}\right)$.

\subsubsection{Dynamic Framework of Resilience and Preparedness}

- Case I $\left(B_{P}>C_{P}\right)$

We begin with the case where benefit of preparedness is greater than the cost. If there was no disruption (see the "No Disruption" branches in Figure 1.2), $B_{P}-C_{P}>$ 0, households should take the preparedness actions to achieve a better outcome (e.g., households who install wind resistant doors and windows may qualify for home insurance discounts irrespective of hurricane events).

We are more interested in situations where households experienced monetary damages from hurricane-induced utility disruptions $\left(C_{D} \neq 0\right)$. Figure 1.3a illustrates the temporal dynamics of both prepared and unprepared households to the consequences of hurricane $\left(t_{0}\right.$ to $\left.t_{1}\right)$ and the recovery from the effects $\left(t_{1}\right.$ to $\left.t_{2}\right)$. As shown, households who were prepared for the storm could result in a higher level of 
well-being, indicated as $W(t)$. They suffer from a disruption of $D_{1}$ and have access to a higher path, $W_{1}(t)$, during the recovery period. Unprepared households will suffer a more severe disruption, $D_{2}$, and recover through the lower path at $W_{2}(t)$.

We assume that the cost or damage of $D_{1}$ equals to $C_{D}$ and the cost of $D_{2}$ equals to $a C_{D} . W_{0}$ is the initial level of household well-being from equation (1.6), and $W(t)$ is the level of household well-being at any time $t$. Also note that $t_{1}$ can be found at any point between $t_{0}$ and $t_{2}$, because the starting points of the recovery process are not uniform among households. Next, we compare the household resilience $(R)$ by integrating the areas under $W_{1}(t)$ and $W_{2}(t)$, to compare the outcomes presented in Figure 1.3a.

The resilience of prepared households $\left(R_{1}\right)$ is measured as:

$$
\begin{gathered}
W_{1}(t)=W_{0}+\left(B_{P}-C_{P}\right)-C_{D} e^{-b_{1} t} \\
R_{1}=\frac{\int_{t_{1}}^{t_{2}}\left[W_{1}(t)\right] d t}{\left(t_{2}-t_{1}\right)}=\frac{\int_{t_{1}}^{t_{2}}\left[W_{0}+\left(B_{P}-C_{P}\right)-C_{D} e^{-b_{1} t}\right] d t}{\left(t_{2}-t_{1}\right)}
\end{gathered}
$$

Similarly, the resilience of unprepared households $\left(R_{2}\right)$ is measured as:

$$
\begin{gathered}
W_{2}(t)=W_{0}-a C_{D} e^{-b_{2} t} \\
R_{2}=\frac{\int_{t_{1}}^{t_{2}}\left[W_{2}(t)\right] d t}{\left(t_{2}-t_{1}\right)}=\frac{\int_{t_{1}}^{t_{2}}\left[W_{0}-a C_{D} e^{-b_{2} t}\right] d t}{\left(t_{2}-t_{1}\right)}
\end{gathered}
$$

where $b_{1}$ is the slope of the $W_{1}(t)$ path and $b_{2}$ is the slope of the $W_{2}(t)$ path. We assume $b_{1}>b_{2}$, because households who take preparation actions may have a higher socio-economic status, which further contributes to a higher speed of recovery. 
Now, $W_{1}(t)-W 2(t)$

$$
\begin{aligned}
& =\left[W_{0}+\left(B_{P}-C_{P}\right)-C_{D} e^{-b_{1} t}\right]-\left[W_{0}-a C_{D} e^{-b_{2} t}\right] \\
& =\left(B_{P}-C_{P}\right)-C_{D} e^{-b_{1} t}+a C_{D} e^{-b_{2} t} \\
& \geq\left(B_{P}-C_{P}\right)-C_{D} e^{-b_{1} t}+a C_{D} e^{-b_{1} t} \\
& =\left(B_{P}-C_{P}\right)+(a-1) C_{D} e^{-b_{1} t}>0 \quad(a>1)
\end{aligned}
$$

Therefore, $W_{1}(t)>W_{2}(t)$ and $R_{1}>R_{2}$, implying that prepared households have access to a higher level of well-being compared to unprepared households. Preparedness activities and socio-economic characteristics can contribute to a higher level of household resilience.

- Case II $\left(B_{P}<C_{P}\right)$

Next, we study the case when the benefit of preparedness is less than the cost. If there was no hurricane-induced disruption, $B_{P}-C_{P}<0$, the net benefit of preparedness becomes negative. Households may not take self-protective actions at such a high cost.

We next examine the situation where households experienced damages from utility disruptions (see Figure 1.3b). Would the households still benefit from hurricane preparation? According to the "Disruption" branches in Figure 1.2, prepared households could yield a better outcome if:

$$
\begin{gathered}
B_{P}-C_{P}-C_{D} \geq-a C_{D} \\
\text { Or, } C_{P}-B_{P} \leq(a-1) C_{D} \quad(a>1)
\end{gathered}
$$


Under this condition, $W_{1}(t)-W_{2}(t)$

$$
\begin{aligned}
& =\left[W_{0}+\left(B_{P}-C_{P}\right)-C_{D} e^{-b_{1} t}\right]-\left[W_{0}-a C_{D} e^{-b_{2} t}\right] \\
& =\left(B_{P}-C_{P}\right)-C_{D} e^{-b_{1} t}+a C_{D} e^{-b_{2} t} \\
& \left.\geq\left(B_{P}-C_{P}\right)-C_{D} e^{-b_{1} t}+a C_{D} e^{-b_{1} t} \quad \text { Assume } b_{1} \geq b_{2}\right) \\
& =\left(B_{P}-C_{P}\right)+(a-1) C_{D} e^{-b_{1} t} \\
& \geq\left(B_{P}-C_{P}\right)+\left(C_{P}-B_{P}\right) e^{-b_{1} t} \\
& =\left(C_{P}-B_{P}\right)\left(e^{-b_{1} t}-1\right)>0
\end{aligned}
$$

Therefore, $W_{1}(t)>W_{2}(t)$ and $R_{1}>R_{2}$, under the condition expressed in (1.13).

Hurricane preparedness can be beneficial to households when its net cost is less than the difference in damages caused by wind-induced utility disruptions. This is especially important for low-income households. Hazard adjustment, such as shutters and other retrofitting processes, often requires significant amount of investment. Hence, income may well be related to household behaviors (Ge, Peacock, \& Lindell, 2011). Due to the fact that many households have limited financial resources to allocate in a disastrous event, policies that lower the costs of their preparedness activities (e.g., insurance premium discounts) can be very useful.

To sum up, the area of household resilience $R$ is determined by the effective preparation at low cost $\left(B_{P}-C_{P}\right)$, the extent of damages caused by hurricaneinduced disruptions $\left(C_{D}\right)$, and the ability to quickly recover $(b)$. Particularly, the speed of recovery $(b)$ is related to households' socio-economic characteristics. Based on this dynamic framework of household resilience, we will introduce the data and empirically test some of these determinants of household resilience. 


\subsection{Data and Sample Characteristics}

The data comes from a household survey designed by researchers at the Social Science Laboratory of the International Hurricane Research Center (IHRC) at Florida International University (FIU). GfK (formerly known as Knowledge Networks), a reputed organization that routinely implements a variety of public opinion surveys, conducted the survey through the internet on behalf of IHRC over a period of two weeks (July 7 - 22, 2013). The targeted population consisted of eligible adults (ages 18 and older) who resided in Hurricane Sandy's most affected areas in ten states. ${ }^{1}$ GfK sampled the households from its KnowledgePanel, a probability-based web panel designed to represent the population of the United States.

Respondents were asked to participate in the survey by two stages (See Figure 1.4). At the first stage, they were asked to report any monetary loss caused by Sandy. Five types of damage were reported: exterior home damage, interior home damage (e.g., walls, ceilings, floors, etc.), damage to furniture, damage to internal contents (e.g., computers, books, jewelry, tools, etc.), and damage to automobiles. At the second stage, only respondents who reported a positive amount on any type of above damages were asked to rate how well they have recovered from the effects of Hurricane Sandy (in a scale of 0 to 10, while 10 indicated a level of "fully recovered" and 0 a level of "not recovered at all"). Because this self-rated level is usually seen as subjective from one individual to another, concerns may arise. For instance, an individual who believed that he or she was almost recovered might report a number of 9 , while the other who thought the same way may only report a number of 6 . To mitigate this possible subjective bias in some extent, we have reclassified the

\footnotetext{
${ }^{1}$ The states include New Jersey (NJ), New York (NY), Connecticut (CT), Maryland (MD), Massachusetts (MA), Virginia (VA), Delaware (DE), Pennsylvania (PA), Rhode Island (RI), and West Virginia (WV).
} 
recovery levels into three categories: (1) not well recovered (original rating from 0 to 5); (2) almost recovered (original rating from 6 to 9); (3) fully recovered (original rating of 10). ${ }^{2}$ Also, we considere households who were fully recovered at the time of the survey as "resilient households" and households who were not well recovered as "fragile households", and those who were almost recovered stand somewhere in between.

We received a full sample of 1,042 respondents in the first stage. Map 1 in Figure 1.5 presents the number and the location of surveyed respondents by state. ${ }^{3}$ As we can see, the majority of them living in the northeastern region and along the east coast were affected by Sandy, with a large proportion from New Jersey (36.56\%) and New York (28.41\%). This is consistent with the fact that Sandy turned into a huge storm with intense winds when it made landfall over New Jersey. As time progressed, Sandy weakened as it moved inland over Pennsylvania to the northeastern states. Among all the respondents, 334 have reported their damages (32.05\%, indicated by red circles) caused by Hurricane Sandy and were asked to participate in the second stage. 150 of them completed the survey (completion rate of 45.51\%). According to Map 2 in Figure 1.5, 121 out of 150 respondents (80.67\%) were from New York or New Jersey. This high proportion further indicates that, these two states were truly the "hot spot" of this event. Even though households from the rest of the states were affected as well, most of them did not suffer in terms of monetary loss. In addition, the recovery level rated by each respondent can be located in Map 2. Approximately

\footnotetext{
${ }^{2}$ No significant differences in the estimation results were found when using the original levels instead of the reclassified three categories. Therefore, we use the three categories for the simplicity of post-estimation analysis in this study.

${ }^{3}$ Figure 1.5 is prepared by using ArcMap 10.2.2 software. The location of each respondent is Geo-coded based on the longitude and latitude information. Coordinates are in GCS North American 1983.
} 
half of them (45.33\%) believed that they "fully recovered" from Hurricane Sandy after eight months. About $37.33 \%$ stated that they "almost recovered" and $17.33 \%$ reported the status "not well recovered".

We utilize three sets of independent variables based on our framework of household resilience. The first set of variables includes five essential utility systems or services: electricity, water, gas, phone/cell phone, and public transportation. Respondents were asked to count how many days they experienced disruptions for each of these services. Figure 1.6 gives a general picture about the percentage and duration of each utility disruption reported by respondents from the full sample. ${ }^{4}$ Among those who lost electricity, the majority reported a disruption for less than a week (of which, $35.73 \%$ reported being without electricity for less than three days and $37.70 \%$ for four to seven days). Around $23.82 \%$ respondents lost electricity for more than a week but less than two weeks (8 - 14 days). A smaller proportion of respondents had to put up with a longer period without electricity: $2.49 \%$ for more than two weeks but less than one month (15 - 30 days) and $0.26 \%$ for more than one month. Similar patterns are found with respect to disruptions in other four utility services, indicating that most households were able to resume normal activities within one week, at most two weeks.

The second set of variables includes self-protective actions and alternative resources undertaken by households for dealing with hurricane impacts. The first variable used is insurance coverage, and respondents were asked if they had an insurance policy that paid for damages to their homes from a storm or hurricane. The second and third variables utilized are window protection and generator. Respon-

\footnotetext{
${ }^{4}$ We did not present the disruption rate of each utility service in Figure 1.6. In fact, $73.32 \%$ of respondents have reported electricity disruptions, $12.76 \%$ have reported water disruptions, $15.26 \%$ have reported gas disruptions, and $46.93 \%$ and $33.97 \%$ have reported phone/cell phone and public transportation disruptions, respectively.
} 
dents were asked if they had any kind of protection (such as storm shutters, security film, or plywood) to protect the windows, and if they owned an electric generator during Hurricane Sandy. More than half of the respondents (61\%) had an insurance policy and $24 \%$ owned an electric generator. However, only $7 \%$ of them installed any kind of window protection in preparation for Sandy.

The third set of variables consists of various household socio-economic characteristics, such as age, education, income, family size, and years of residence. We also include past hurricane experience in the analysis. The respondents' average age was 53 years old, and the average household size was 2.49. The average year of residence was 17 - 18 years. About 12.57\% of the respondents had high school diploma or less, and $59.98 \%$ of them had a Bachelor's degree or higher. The average annual income level ranged from $\$ 60,000$ to $\$ 75,000$. Sixty-eight percent of the respondents had hurricane experience as they indicated that they were living in an area impacted by Hurricane Irene in $2011 .^{5}$

Apart from utilizing the survey responses, we have also measured the characteristics of Hurricane Sandy by using HAZUS-MH (Hazard U.S. - Multi-Hazard) Hurricane Model in order to control for the intensity of the hurricane. ${ }^{6}$ The strength of hurricanes is usually measured by wind speed. The model uses wind engineering principles and generates validated wind speeds through comparison of simulated and observed wind speeds. We have first provided necessary inputs to build the Hurricane Sandy scenario in the model. The inputs are taken from National Hur-

\footnotetext{
${ }^{5}$ Hurricane Irene (2011) was one of the most damaging hurricanes prior to Sandy that made landfall in the New York and New Jersey areas.

${ }^{6}$ HAZUS is a geographic information system (GIS) based disaster modeling tool, which is developed and freely distributed by the Federal Emergency Management Agency (FEMA). For technical details, see: http://www.fema.gov/media-library-data/20130726 -1820-25045-8522/hzmh2_1_hr_um.pdf
} 
ricane Center's North Atlantic Hurricane Database (HURDAT), which consists of six-hourly positions (latitude, longitude, translation speed, and time) and corresponding intensity estimates (radius to hurricane winds, maximum wind speed, and central pressure) of Sandy. Then, the model is implemented to generate Sandy's track and its maximum sustained wind speeds at the census tract level. Finally, the estimation of wind speed experienced for each respondent can be obtained by spatially joining the respondents' locations into the model. The description of all variables used is presented in Table 1.1. The detailed statistics of each variable on the full sample (from the first stage) and the subsample (from the second stage) can be found in Table 1.2 .

\subsection{Econometric Specification}

As described in section 1.3, the area of household resilience $R$ is determined by the extent of damages caused by hurricane-induced disruptions, the effective preparation, and the ability to quickly recover. To test these determinants of household-level resilience, the following model is estimated:

$$
R_{i}=f(D, P, H)
$$

where $R$ is resilience measured by the level of recovery of household $i$. The variables included in vector $D$ consist of five types of utility disruption experienced by households. The variables included in vector $P$ and vector $H$ consist of preparedness activities and household socio-economic characteristics, respectively.

Although our full sample has been adjusted (using the panel weight method by GfK), we recognize the nonrandom selection aspect of the sample. Due to the fact that our analysis at the second stage is restricted to households who reported mon-

etary damages, the sample selection bias may be present (Heckman, 1979). More 
specifically, we compare the recovery performance of households who experienced longer disruptions to those who experienced shorter disruptions. The group of households who suffered higher damages is included in our sample because they had longer disruptions, and they will be representative of the group with longer disruptions. However, the subset of households with zero damage will not be representative of the group of households with short disruptions. Some households who experienced longer disruptions may still report zero damage due to their preventative actions. Ultimately, it may appear that households with longer disruptions have a higher recovery level with zero damage. In that case we may overestimate the effects of utility disruptions and neglect the benefits of hurricane preparation.

For this reason, we need to employ a technique that controls for the sample selection bias. We also need to extend the standard selection model to accommodate ordinality, as the recovery level studied in our model is an ordinal variable (Greene, 2008). Miranda and Rabe-Hesketh (2006) suggested that consistent estimators can be obtained by maximum likelihood estimation of a joint model of the selection and outcome variables. To account for the sample selection issue and ordinal feature of the recovery level, we employ an ordered logit regression with sample selection.

First, the equation of the selection variable is specified as:

$$
R_{1 i}^{*}=\beta_{1} D+\delta_{1} P+\gamma_{1} H+\varepsilon_{1 i}
$$

where $R_{1 i}^{*}$ is the unobserved variable represented by $R_{1 i}$ with the following structure:

$$
R_{1 i}= \begin{cases}0, & \text { if } \quad R_{1 i}^{*}=0 \\ 1, & \text { if } \quad R_{1 i}^{*}>0\end{cases}
$$

$R_{1 i}^{*}$ is a continuous variable to measure the households' monetary damages resulted from Hurricane Sandy. $D, P$ and $H$ are vectors of explanatory variables from equation (1.15). $\beta_{1}, \delta_{1}$ and $\gamma_{1}$ are the vectors of corresponding coefficients. 
Next, the equation of the outcome variable is specified as:

$$
R_{2 i}^{*}=\beta_{2} D+\delta_{2} P+\gamma_{2} H+\varepsilon_{2 i}
$$

where $R_{2 i}^{*}$ is the unobserved variable represented by $R_{2 i}$ with the following structure:

$$
R_{2 i}=\left\{\begin{array}{ccc}
1 \Rightarrow N R & (\text { Not Recovered }), & \text { if } \tau_{0}=-\infty \leq R_{2 i}^{*}<\tau_{1} \\
2 \Rightarrow A R & (\text { Almost Recovered }), & \text { if } \tau_{1} \leq R_{2 i}^{*}<\tau_{2} \\
3 \Rightarrow F R & (\text { Fully Recovered }), & \text { if } \tau_{2} \leq R_{2 i}^{*}<\tau_{3}=\infty
\end{array}\right.
$$

where $R_{2 i}^{*}$ is a continuous variable of the level of recovery, and $R_{2 i}$ is the rated recovery level for the $i$ th household to present the continuous scale. Note that $R_{2 i}$ is observed only if the selection condition $\left(R_{1 i}=1\right)$ from equation (1.17) is met, and as before, $\beta_{2}, \delta_{2}$ and $\gamma_{2}$ are the vectors of corresponding coefficients. We expect $\beta_{2}$ to be negative in our model. Also, $\varepsilon_{2 i}$ are the standard normal random errors, and $\tau_{j}$ s are alternative specific constants. We assume that $\varepsilon_{1 i}$ in equation (1.16) and $\varepsilon_{2 i}$ follow a bivariate standard normal distribution with a correlation $\rho$. The potential sample selection bias can be identified by testing whether $\rho$ is statistically different from zero (Greene, 2008). If the null hypothesis $\rho=0$ can not be rejected, the selection bias is not a major concern, and we can proceed by using the usual ordered logit regression.

The probability of an observed recovery outcome $m$ for a given value of $D_{i}, P_{i}$ or $H_{i}$ is given as (Long, 1997):

$$
\operatorname{Pr}\left(R_{2 i}=m \mid D_{i}, P_{i}, \text { or } H_{i}\right)=\operatorname{Pr}\left(\tau_{m} \leq R_{2 i}^{*}<\tau_{m+1} \mid D_{i}, P_{i}, \text { or } H_{i}\right)
$$

We can estimate the model to obtain the probabilities of observing each of the three recovery levels. They are given as:

$$
\operatorname{Pr}\left(R_{2 i}=N R\right)=F\left(\tau_{1}-\beta_{2} D_{i}-\delta_{2} P_{i}-\gamma_{2} H_{i}\right)
$$




$$
\begin{gathered}
\operatorname{Pr}\left(R_{2 i}=A R\right)=F\left(\tau_{2}-\beta_{2} D_{i}-\delta_{2} P_{i}-\gamma_{2} H_{i}\right) \\
-F\left(\tau_{1}-\beta_{2} D_{i}-\delta_{2} P_{i}-\gamma_{2} H_{i}\right) \\
\operatorname{Pr}\left(R_{2 i}=F R\right)=1-F\left(\tau_{2}-\beta_{2} D_{i}-\delta_{2} P_{i}-\gamma_{2} H_{i}\right)
\end{gathered}
$$

where $F($.$) is the cumulative normal distribution function.$

Now, we can study the effect of each variable by measuring the change in the outcome probabilities, holding all other variables constant. The marginal effect for each utility disruption can be computed as (Long, 1997):

$$
\begin{aligned}
& \frac{\partial \operatorname{Pr}\left(R_{2 i}=m \mid D_{i}\right)}{\partial D_{i k}}=\frac{\partial F\left(\tau_{m+1}-\beta_{2} D_{i}\right)}{\partial D_{i k}}-\frac{\partial F\left(\tau_{m}-\beta_{2} D_{i}\right)}{\partial D_{i k}} \\
& \frac{\partial \operatorname{Pr}\left(R_{2 i}=m \mid P_{i}\right)}{\partial P_{i k}}=\frac{\partial F\left(\tau_{m+1}-\beta_{2} P_{i}\right)}{\partial P_{i k}}-\frac{\partial F\left(\tau_{m}-\beta_{2} P_{i}\right)}{\partial P_{i k}} \\
& \frac{\partial \operatorname{Pr}\left(R_{2 i}=m \mid H_{i}\right)}{\partial H_{i k}}=\frac{\partial F\left(\tau_{m+1}-\beta_{2} H_{i}\right)}{\partial H_{i k}}-\frac{\partial F\left(\tau_{m}-\beta_{2} H_{i}\right)}{\partial H_{i k}}
\end{aligned}
$$

\subsection{Estimation Results}

The ordered logit regressions are estimated by using maximum likelihood methods, and Table 1.3 presents the estimation results. Columns (1) and (2) report the estimation results with sample selection method, by using the joint maximum likelihood estimation of household damage and recovery level. ${ }^{7}$ The cross-equation correlation $\rho$ of estimated errors of the selection variable (damage) and the outcome variable (recovery) is used to test for sample selection bias. As a result, the likelihood ratio test for null hypothesis of uncorrelated error $(\rho=0)$ cannot be rejected $(p=0.978)$, suggesting that the samples in the second stage are randomly selected with no selection bias. Therefore, we can obtain consistent estimators by estimating the two

\footnotetext{
${ }^{7}$ The generalized linear latent and mixed model (GLLAMM) with the ssm command is used in STATA to estimate the ordered logit model with sample selection.
} 
regression equations sequentially at both stages. ${ }^{8}$ Column (3) reports the logit regression on hurricane-induced damage for all households from the first stage. The rest of the columns present four models predicting household-level recovery using ordered logit regressions, with selected households from the second stage.

As expected, wind speed has a significant and strong positive impact on damage (see column (3)). The predicted probabilities of reporting positive damages by respondents at different wind speeds are presented in Figure 1.7. The positive effect is evident by the increasingly larger probabilities on damage as wind speed increases (from 0.14 when wind speed is 50 miles per hour to 0.55 when wind speed is 95 miles per hour). The $95 \%$ confidence intervals around the predictions are also added, which are smaller near the center of the data where wind speed is around 75 miles per hour, and increase as we move to lower or higher wind speeds.

We then examine the effects of utility disruptions on households' activities. According to Table 1.3, disruptions in electricity, water, gas, and public transportation had significant impacts during Hurricane Sandy. A longer duration of disruptions of these utilities increases the possibility of incurring damages at the first stage. Also, electricity outage reveals a stronger impact on damage than disruptions in other utilities, indicated by a larger coefficient. The failure of power supply also significantly affects the supply of other utility services, implying the cascading effects.

In terms of recovery performance, disruptions in electricity, water, gas and phone are found to be statistically significant with expected negative signs. Households who experienced longer days of these disruptions are less likely to have a higher level of resilience. As shown in column (5) of Table 1.3, the utility disruption model accounts for $15.3 \%$ of the variance, explaining the differential recovery outcomes very

\footnotetext{
${ }^{8}$ Note that there are no substantial differences on the estimation results between ordered logit regression with and without sample selection method.
} 
well. It is also useful to interpret the results by computing the marginal effect on each level of recovery (see Table 1.4). Water supply is identified as the most influencing utility service at the recovery stage, indicated by the larger marginal effect. Without a doubt, water is an essential element to survival. In the aftermath of a hurricane, it is critical to have sufficient clean water for household consumption, maintaining basic hygiene and resuming normal activities. According to Table 1.4, the marginal effect of water is 0.0131 in the NR column, indicating that each additional day of water disruption increases the probability of being "not well recovered" by $1.31 \%$. On the other hand, the marginal effect of water is -0.0318 in the FR column, indicating that each additional day of water disruption decreases the probability of being "fully recovered" by $3.18 \%$.

Figure 1.8 graphically displays the predicted probabilities on the three recovery outcomes. The computed values are reported in Table 1.5. Overall, the patterns of each recovery level are shown to be very similar across different types of utility disruption. The likelihood of being not well recovered (NR) increases with longer days of disruptions, while the likelihood of being fully recovered (FR) decreases. Again, water supply displays the most pronounced effect. The predicted probability of "fully recovered", indicated by the line with squares, is 0.425 for households who never lost water supply. However, the probability decreases rapidly to 0.045 at 20 days without water and to almost 0 at 50 days. The predicted probability of "not well recovered", indicated by the line with circles, is nearly the mirror image. It begins at 0.086 at no disruption and ends at 0.999 at 50 days of disruptions. The probability of "almost recovered" is nonlinear, as indicated by the line with triangles. It begins at 0.489 , increases to 0.571 , and then decreases to almost 0.001 . The effect of water disruption on "almost recovered" is initially positive and then negative. This occurs because as days increase without water supply, more households are 
likely to move from category FR to AR than move from AR to NR; consequently the probability of AR increases. When the days of water disruption become longer, more households leave AR for NR than enter AR from FR, resulting in a decrease in probability.

Next, we examine the role of household characteristics in explaining the determinants of households' damages and recovery performance. The preparedness model accounts for $6.5 \%$ of the variance of recovery outcome, according to column (6) in Table 1.3. Having an insurance policy is found to have a positive effect on household recovery. It is, however, also positively significant for the damage equation. This is possible because people who hold insurance to cover disasters usually live in an area where frequent disaster occurs. Another possible explanation is that if households realized that they can have their damages covered during a hurricane, they may overestimate the damages and put less efforts in preventing their properties due to the "moral hazard" problem.

Window protection is positively significant in both damage and recovery, indicating that households who installed window protections are more likely to report monetary damages, but are also more likely to recover rapidly from those damages. This is possible because households who had window protections may reside in an area severely impacted by Sandy. However, engaging in self-protective actions may also contribute to a higher level of resilience. Figure 1.9 presents the predicted probabilities on household recovery with and without window protection. As indicated, prepared households $\left(R_{1}\right)$ are more likely to achieve the "fully recovered" level compared to unprepared households $\left(R_{2}\right)$, providing empirical supports to our framework. Purchasing an electric generator is found to be positively significant. Households who had a generator are quicker in recovering. As shown in Figure 1.10, owning a generator in preparing for Sandy has contributed to a higher probability 
of "fully recovered" level for prepared households $\left(R_{1}\right)$, and unprepared households $\left(R_{2}\right)$ yield a lower probability due to their lack of self-protective behaviors.

Socio-economic characteristics are found to be important determinants of household damage and recovery. Consistent with the literature, households with elderly and a larger family size and longer years of residence are more likely to report damages caused by Sandy. Educational attainment is found to be significant and positive on both stages. An educated household is more likely to have suffered from damages (due to wealth effect), but is also more likely to recover from those damages quickly.

Past experience from Hurricane Irene in 2011 also plays a role in prompting households to a higher recovery level. This result is in line with previous findings that experience and knowledge are important factors. Table 1.6 presents the predicted probabilities of each recovery level by different types of households, based on their hurricane experience and education, while holding other characteristics constant at mean. If we look at only households without past hurricane experience (Irene = 0), their recovery level differs with educational attainments. Households with an advanced bachelor's degree or higher have a higher likelihood of being "resilient households". We can also examine the role of hurricane experience. For example, the likelihood of being "fully recovered" is increased, and the likelihood of being "not well recovered" is decreased, by gaining hurricane experience (from Irene = 0 to Irene $=1$ ). Among all types of household, the highest predicted probability (0.525) of being a "resilient household" is associated with those who had a bachelor's degree or higher and experienced a hurricane. On the opposite, the highest predicted probability (0.571) of being a "fragile household" is associated with those who had less than a high school degree and had no hurricane experience.

Overall, we have found evidence to support that utility disruptions are indeed important determinants of long-term household recovery. The findings from the full 
model further suggest that even after controlling for household characteristics, utility disruptions have significant effects. Just as important, the effects of preparedness activities are positive, indicating that effective self-protective actions and available alternative resources can improve household resilience. Furthermore, households' ability to quickly recover is associated with socio-economic characteristics, such as education and past hurricane experience. These empirical findings from regression analyses therefore substantiate our dynamic framework of household resilience.

\subsection{Conclusion}

A disastrous event like Hurricane Sandy may not be treated as one occasional incident but rather one that we should expect more frequently due to the exacerbated impact of climate change and sea level rise (Knutson et al., 2010; Nicholls \& Cazenave, 2010). Special attention should be given to understand hurricane impacts and their consequences, including the household-level recovery processes. This study presents a detailed analysis on the determinants of household recovery in the aftermath of Hurricane Sandy. We use responses collected through a household survey, which households were first asked to report damages in monetary terms. Households who reported a positive amount of damages were asked to rate their recovery levels at the second stage. Given that we did not find evidence of selection bias, the hurricane-induced damage and level of recovery are estimated sequentially. The estimation results on the sequential models further confirm that the determinants of damage are not necessarily the same that drive recovery performance.

The major findings suggest that Hurricane Sandy had long-lasting effects on households in the affected areas, especially in the state of New York and New Jersey. Those who suffered from longer utility disruptions are more likely to report 
hurricane-induced damages and have more difficulty in recovering. By computing the marginal effects and predicted probabilities, we find that electricity disruption has the largest effect that increases the likelihood of household damages, and water supply is the most critical utility service at the recovery stage. Effective preparedness engagements, such as installing window protection and purchasing generators, can have positive results in reducing adverse shocks. Households with past hurricane experiences and higher educational attainments are associated with faster recovery performance. Our results are robust with or without the inclusion of the sample selection method.

One major contribution of this study is that we explore the role of utility disruption on household-level recovery, based on an extended framework of disaster resilience. This topic has not been sufficiently covered in the previous literature. The results shed light on the fact that public utility supply during a hurricane is crucial to households in the affected areas. Policy attention is warned for ensuring rapid restoration of infrastructures to reduce the negative impacts after disastrous events. Our findings also suggest that policy actions targeted to influence self-protective behaviors, such as insurance premium discounts or discounts on alternative resources (i.e., offering discounts for purchasing generators in hurricane prone areas), can be useful. Also, sharing analysis-based information from previous hurricanes can be utilized to educate self-protecting behaviors (attending disaster preparation programs, applying for post-disaster assistance, etc.) to enable households better cope with disasters. Furthermore, locating the most vulnerable people (such as elderly and large family and ethnic minority groups) within communities is an important step toward effective disaster management. Finally, we hope that our findings contribute to the limited empirical evidence on the impact of utility disruptions in the natural hazard literature and provide useful insights for promoting community resilience. 


\section{References}

Alinovi, L., D’Errico, M., Mane, E., \& Romano, D. (2010). Livelihoods strategies and household resilience to food insecurity: An empirical analysis to Kenya. Paper presented at the Conference on "Promoting Resilience through Social Protection in Sub-Saharan Africa", Dakar, Senegal. Avai:

http://erd.eui.eu/media/BackgroundPapers/Alinovi-Romano-D'Errico-Mane.pdf

Berke, P. R., Kartez, J., \& Wenger, D. (1993). Recovery after disaster: Achieving sustainable development, mitigation and equity. Disasters, 17(2), 93-109. doi: 10.1111/j.1467-7717.1993.tb01137.x

Botzen, W. J. W. (2013). Managing extreme climate change risks through insurance. New York \& Cambridge: Cambridge University Press.

Breed, A. G., \& Hays, T. (2012, November 15). Hurricane Sandy aftermath: Storm leaves millions without power, dozens dead. The Huffington Post. Available online:http://www.huffingtonpost.com/2012/10/30/

hurricane-sandy-storm_n_2042815.html

Bruneau, M., Chang, S. E., Eguchi, R. T., Lee, G. C., O’Rourke, T. D., Reinhorn, A. M., von Winterfeldt, D. (2003). A framework to quantitatively assess and enhance the seismic resilience of communities. Earthquake Spectra, 19(4), 733-752. doi: $10.1193 / 1.1623497$

Carlsson, F., Martinsson, P., \& Akay, A. (2011). The effect of power outages and cheap talk on willingness to pay to reduce outages. Energy Economics, 33(5), 790-798. doi: http://dx.doi.org/10.1016/j.eneco.2011.01.004

Chang, S. E., McDaniels, T., Fox, J., Dhariwal, R., \& Longstaff, H. (2014). Toward disaster-resilient cities: Characterizing resilience of infrastructure systems with expert judgments. Risk Analysis, 34(3), 416-434. doi: 10.1111/risa.12133

Chang, S. E., \& Miles, S. (2004). The dynamics of recovery: A framework. In Y. Okuyama, \& S. Chang (Eds.), pp. 181-204, Springer Berlin Heidelberg.

Chang, S. E., Svekla, W. D., \& Shinozuka, M. (2002). Linking infrastructure and urban economy: Simulation of water-disruption impacts in earthquakes. Environment and Planning B: Planning and Design, 29(2), 281-301. doi:10.1068/b2789 
Chatterjee, C., \& Mozumder, P. (2015). Hurricane Wilma, utility disruption, and household wellbeing. International Journal of Disaster Risk Reduction, 14(4), 395-402. doi: http://dx.doi.org/10.1016/j.ijdrr.2015.09.005

Cutter, S. L., Boruff, B. J., \& Shirley, W. L. (2003). Social vulnerability to environmental hazards. Social Science Quarterly, 84(2), 242-261.

doi:10.1111/1540-6237.8402002

Ewing, B. T., Kruse, J. B., \& Sutter, D. (2007). Hurricanes and economic research: An introduction to the hurricane Katrina symposium. Southern Economic Journal, $74(2), 315-325$.

Faupel, C. E., \& Styles, S. P. (1993). Disaster education, household preparedness, and stress responses following Hurricane Hugo. Environment and Behavior, 25(2), 228-249. doi: 10.1177/0013916593252004

Fothergill, A., \& Peek, L. (2004). Poverty and disasters in the United States: A review of recent sociological findings. Natural Hazards, 32(1), 89-110. doi: 10.1023/B:NHAZ.0000026792.76181.d9

Frankenberg, E., Gillespie, T., Preston, S., Sikoki, B., \& Thomas, D. (2011). Mortality, the family and the Indian Ocean tsunami. The Economic Journal, 121 (554), 162-182. doi: 10.1111/j.1468-0297.2011.02446.x

Fronstin, P., \& Holtmann, A. G. (1994). The determinants of residential property damage caused by hurricane Andrew. Southern Economic Journal, 61(2), 387-397. doi: $10.2307 / 1059986$

Ge, Y., Peacock, W. G., \& Lindell, M. K. (2011). Florida households' expected responses to hurricane hazard mitigation incentives. Risk Analysis, 31(10), 1676-1691. doi: 10.1111/j.1539-6924.2011.01606.x

Glewwe, P., \& Hall, G. (1998). Are some groups more vulnerable to macroeconomic shocks than others? Hypothesis tests based on panel data from Peru. Journal of Development Economics, 56(1), 181-206. doi: http://dx.doi.org/10.1016/S0304-3878(98)00058-3

Greene, W. H. (2008). Econometric analysis (6th Ed.). Upper Saddle River, N.J: Pearson/Prentice Hall.

Griffin, R. C., \& Mjelde, J. W. (2000). Valuing water supply reliability. American Journal of Agricultural Economics, 82(2), 414-426. doi: 10.1111/0002-9092.00035 
Hallstrom, D. G., \& Smith, V. K. (2005). Market responses to hurricanes. Journal of Environmental Economics and Management, 50(3), 541-561. doi:

http://dx.doi.org/10.1016/j.jeem.2005.05.002

Han, S., Guikema, S. D., \& Quiring, S. M. (2009). Improving the predictive accuracy of hurricane power outage forecasts using generalized additive models. Risk Analysis, 29(10), 1443-1453. doi: 10.1111/j.1539-6924.2009.01280.x

Heckman, J. J. (1979). Sample selection bias as a specification error. Econometrica, 47(1), 153-161.

Howe, C. W., Smith, M. G., Bennett, L., Brendecke, C. M., Flack, J. E., Hamm, R. M., . . . Wunderlich, K. (1994). The value of water supply reliability in urban water systems. Journal of Environmental Economics and Management, 26(1), 19-30. doi: http://dx.doi.org/10.1006/jeem.1994.1002

Knutson, T. R., McBride, J. L., Chan, J., Emanuel, K., Holland, G., Landsea, C., \& Sugi, M. (2010). Tropical cyclones and climate change. Nature Geosci, 3(3), 157-163.

Liu, H., Davidson, R., Rosowsky, D., \& Stedinger, J. (2005). Negative binomial regression of electric power outages in hurricanes. Journal of Infrastructure Systems, 11 (4), 258-267. doi: 10.1061/(ASCE)1076-0342(2005)11:4(258)

Long, J. S. (1997). Regression models for categorical and limited dependent variables. Thousand Oaks, Calif: Sage Publications.

Marshall, M., \& Schrank, H. (2014). Small business disaster recovery: A research framework. Natural Hazards, $72(2)$, 597-616. doi:10.1007/s11069-013-1025-z

Masozera, M., Bailey, M., \& Kerchner, C. (2007). Distribution of impacts of natural disasters across income groups: A case study of New Orleans. Ecological Economics, 63(23), 299-306. doi: http://dx.doi.org/10.1016/j.ecolecon.2006.06.013

Mddonnell, S., Troiano, R. P., Barker, N., Noji, E., Hlady, W. G., \& Hopkins, R. (1995). Evaluation of long-term community recovery from Hurricane Andrew: Sources of assistance received by population sub-groups. Disasters, 19(4), 338-347. doi: $10.1111 / \mathrm{j} .1467-7717.1995 . t b 00354 . x$

Mileti, D. S. (1999). Disasters by design: A reassessment of natural hazards in the United States. Washington, D.C: Joseph Henry Press. 
Miranda, A., \& Rabe-Hesketh, S. (2006). Maximum likelihood estimation of endogenous switching and sample selection models for binary, ordinal, and count variables. Stata Journal, 6(3), 285-308.

Morris, S. S., Neidecker-Gonzales, O., Carletto, C., Mungua, M., Medina, J. M., \& Wodon, Q. (2002). Hurricane Mitch and the livelihoods of the rural poor in Honduras. World Development, 30(1), 49-60. doi:

http://dx.doi.org/10.1016/S0305-750X(01)00091-2

Morrow, B. H. (1999). Identifying and mapping community vulnerability. Disasters, 23(1), 1-18. doi: 10.1111/1467-7717.00102

Mozumder, P., Bohara, A.K., Berrens, R.P., \& Halim, N. (2009). Private transfers to cope with a natural disaster: Evidence from Bangladesh. Environment and Development Economics, 14(2), 187-210. doi: 10.1017/S1355770X08004804

Murphy, B. L. (in press). Financial recovery from disasters at the household level: Canadian approaches. Disasters and Emergency Management in Canada, online-textbook (Canadian Risks and Hazards Network.) Available online: http://www.crhnet.ca/sites/default/files/library/Murphy.pdf

Nicholls, R. J., \& Cazenave, A. (2010). Sea-level rise and its impact on coastal zones. Science, 328(5985), 1517-1520.

Nigg, J. M. (1995). Business disruption due to earthquake-induced lifeline interruption. University of Delaware Disaster Research Center. Preliminary Paper No.220. Available online:

http://dspace.udel.edu/bitstream/handle/19716/626/PP220.pdf?sequence=1

Peacock, W. G. (2003). Hurricane mitigation status and factors influencing mitigation status among Florida's single-family homeowners. Natural Hazards Review, 4(3), 149-158. doi: 10.1061/(ASCE)1527-6988(2003)4:3(149)

Peacock, W. G., Brody, S. D., \& Highfield, W. (2005). Hurricane risk perceptions among Florida's single family homeowners. Landscape and Urban Planning, 73(23), 120-135. doi: http://dx.doi.org/10.1016/j.landurbplan.2004.11.004

Reed, D. A., Kapur, K. C., \& Christie, R. D. (2009). Methodology for assessing the resilience of networked infrastructure. Systems Journal, IEEE, 3(2), 174-180. doi: 10.1109/JSYST.2009.2017396 
Reed, D. A. (2008). Electric utility distribution analysis for extreme winds. Journal of Wind Engineering and Industrial Aerodynamics, 96(1), 123-140. doi: http://dx.doi.org/10.1016/j.jweia.2007.04.002

Resurreccion, J., \& Santos, J. (2013). Uncertainty modeling of hurricane-based disruptions to interdependent economic and infrastructure systems. Natural Hazards, 69 (3), 1497-1518. doi: 10.1007/s11069-013-0760-5

Rose, A., \& Liao, S. (2005). Modeling regional economic resilience to disasters: A computable general equilibrium analysis of water service disruptions. Journal of Regional Science, 45(1), 75-112. doi: 10.1111/j.0022-4146.2005.00365.x

Rose, A., \& Lim, D. (2002). Business interruption losses from natural hazards: Conceptual and methodological issues in the case of the Northridge earthquake. Global Environmental Change Part B: Environmental Hazards, 4(1), 1-14. doi: http://dx.doi.org/10.1016/S1464-2867(02)00012-8

Sawada, Y. (2007). The impact of natural and manmade disasters on household welfare. Agricultural Economics, 37, 59-73. doi: 10.1111/j.1574-0862.2007.00235.x

Schultz, T. W. (1975). The value of the ability to deal with disequilibria. Journal of Economic Literature, 13(3), 827-846.

Sledge, M. (2012, November 5). Hurricane sandy leaves 1.3 million still without power as nor'easter approaches. The Huffington Post. Available online: http://www.huffingtonpost.com/2012/11/05/ hurricane-sandy-power-outages_n_2077407.html

Smith, A., \& Maglio, T. (2012, November 1). After Sandy, gas lines stretch for miles. CNNMoney. Available online: http://money.cnn.com/2012/11/01/news/ economy/gas-stations-supply-sandy/index.html

Takashi, K. (2012). Household-level recovery after floods in a developing country: Evidence from Pakistan. CEI Working Paper Series, No 2012-08. Available online: http://hermes-ir.lib.hit-u.ac.jp/rs/bitstream/10086/25637/1/wp2012-8.pdf

Tierney, K. J., \& Nigg, J. M. (1995). Business vulnerability to disaster related lifeline disruption. University of Delaware Disaster Research Center. Preliminary Paper No.223. Available online:

http://udspace.udel.edu/bitstream/handle/19716/631/PP223.pdf?sequence=1 
Tierney, K. J., Lindell, M. K., \& Perry, R. W. (2001). Facing the unexpected: Disaster preparedness and response in the United States. Washington, D.C: Joseph Henry Press.

van Bastelaer, T. (2014). Empowering vulnerable populations to build resilience. Abt Associates thought leadership paper. Available online: http://abtassociates.com/AbtAssociates/files/b2/ b2030353-8f97-4aae-8a28-885b598161db.pdf

Vogel, R. M. (2001). Disaster impact upon urban economic structure: Linkage disruption and economic recovery. In A. Farazmand (Ed.), Handbook of crisis and emergency management, pp. 69-90. New York: Marcel Dekker.

Vogel, R. M. (2002). Lost consumer surplus from natural disaster. Paper presented at the Southwestern Economic Proceedings 2000 Meeting, San Antonio, Texas. Available online:

http://www.ser.tcu.edu/2001-Pro/SEP2001 \%20Vogel $\% 2067-74 . p d f$

Zobel, C. W. (2011). Representing the multi-dimensional nature of disaster resilience. The 8th International ISCRAM Conference, Lisbon, Portugal, May 8-11. Available online:

http://www.iscramlive.org/ISCRAM2011/proceedings/papers/191.pdf 


\section{Tables}

Table 1.1: Description of Variables

Description

Explained variables

Damage How much have you lost due to hurricane Sandy? (in dollars)

Recovery In what extent your household recovered from the effects of hurricane

Sandy? ( $1=$ Not recovered; $2=$ Almost recovered; $3=$ Fully recovered $)$

\footnotetext{
Explanatory variables

Wind $\quad$ Maximum sustained wind speed (miles per hour)

Electricity How many days did you experience disruptions in electricity?

Water

How many days did you experience disruptions in water?

Gas

How many days did you experience disruptions in gas?

Phone

How many days did you experience disruptions in phone/cell phone?

Trans

How many days did you experience disruptions in transportation?

Insurance

Did you have an insurance policy that paid for damages to your home from a hurricane? $(1=$ yes, $0=$ no $)$

Protection Did your home have any kind of window protection such as storm shutters, security film, or plywood to protect the windows during hurricane Sandy? (1=yes, $0=$ no)

Generator Do you or a household member own an electric generator? ( $1=\mathrm{yes}, 0=\mathrm{no})$

Irene

Were you living in an area impacted by Hurricane Irene? (1=yes, $0=$ no)

Age

Age of households in years

Education Highest level of education (1= Less than high school 2= High school; $3=$

Some college; 4= Bachelor's degree or higher)

Income Level of household income

Size Number of people lived in the household

Years Number of years lived at the address

Notes: The income levels are: $1=$ Less than $\$ 5,000 ; 2=\$ 5,000$ to $\$ 7,499 ; 3=\$ 7,500$ to $\$ 9,999 ; 4=\$ 10,000$ to $\$ 12,499 ; 5=\$ 12,500$ to $\$ 14,999 ; 6=\$ 15,000$ to $\$ 19,999 ; 7=$ $\$ 20,000$ to $\$ 24,999 ; 8=\$ 25,000$ to $\$ 29,999 ; 9=\$ 30,000$ to $\$ 39,999 ; 10=\$ 35,000$ to $\$ 39,999 ; 11=\$ 40,000$ to $\$ 49,999 ; 12=\$ 50,000$ to $\$ 59,999 ; 13=\$ 60,000$ to $\$ 74,999$; $14=\$ 75,000$ to $\$ 84,999 ; 15=\$ 85,000$ to $\$ 99,999 ; 16=\$ 100,000$ to $\$ 124,999 ; 17=$ $\$ 125,000$ to $\$ 149,999 ; 18=\$ 150,000$ to $\$ 174,999 ; 19=\$ 175,000$ or more ;
} 
Table 1.2: Descriptive Statistics of Variables in the Sample

\begin{tabular}{|c|c|c|c|c|c|c|c|c|}
\hline \multirow{2}{*}{$\begin{array}{l}\text { Group } \\
\text { Variable }\end{array}$} & \multicolumn{4}{|c|}{$\begin{array}{c}\text { Full Sample }(\mathrm{N}=1042) \\
\text { (First Stage) }\end{array}$} & \multicolumn{4}{|c|}{$\begin{array}{c}\text { Subsample }(\mathrm{N}=150) \\
\quad(\text { Second Stage })\end{array}$} \\
\hline & Mean & SD & Min & Max & Mean & SD & Min & Max \\
\hline Damage & 0.32 & 0.47 & 0 & 1 & 1 & 0 & 1 & 1 \\
\hline Recovery & 2.28 & 0.74 & 1 & 3 & 2.28 & 0.74 & 1 & 3 \\
\hline Wind & 71.34 & 7.64 & 50 & 95 & 73.99 & 7.13 & 51 & 92 \\
\hline Electricity & 4.28 & 5.08 & 0 & 70 & 7.85 & 8.62 & 0 & 70 \\
\hline Water & 0.68 & 2.78 & 0 & 60 & 1.77 & 6.02 & 0 & 60 \\
\hline Gas & 1.10 & 3.89 & 0 & 60 & 2.76 & 8.14 & 0 & 60 \\
\hline Phone & 2.33 & 4.40 & 0 & 60 & 4.48 & 7.01 & 0 & 60 \\
\hline Trans & 1.73 & 3.99 & 0 & 50 & 2.68 & 5.72 & 0 & 50 \\
\hline Insurance & 0.61 & 0.49 & 0 & 1 & 0.82 & 0.39 & 0 & 1 \\
\hline Protection & 0.07 & 0.25 & 0 & 1 & 0.22 & 0.42 & 0 & 1 \\
\hline Generator & 0.24 & 0.43 & 0 & 1 & 0.33 & 0.47 & 0 & 1 \\
\hline Irene & 0.68 & 0.47 & 0 & 1 & 0.69 & 0.47 & 0 & 1 \\
\hline Age & 53.39 & 15.17 & 18 & 91 & 56.29 & 13.80 & 19 & 85 \\
\hline Education & 3.46 & 0.74 & 1 & 4 & 3.50 & 0.65 & 2 & 4 \\
\hline Income & 13.40 & 4.04 & 1 & 19 & 14.20 & 3.61 & 3 & 19 \\
\hline Size & 2.49 & 1.27 & 1 & 10 & 2.57 & 1.34 & 1 & 10 \\
\hline Years & 17.15 & 14.02 & 0 & 68 & 21.11 & 14.75 & 0 & 68 \\
\hline
\end{tabular}


Table 1.3: Estimation Results for Household Damage and Recovery

\begin{tabular}{|c|c|c|c|c|c|c|c|}
\hline & $\begin{array}{l}\text { Sample Sel } \\
\underline{\text { Model }} \\
\text { (1)Dam }\end{array}$ & ection & $\begin{array}{l}\text { Full Mode } \\
\text { (Sequenti } \\
\text { (3)Dam }\end{array}$ & (4)Rec & $\begin{array}{l}\underline{\text { Utility }} \\
\underline{\text { Disruption }} \\
\underline{\text { Model }} \\
(5) \operatorname{Rec}\end{array}$ & $\begin{array}{l}\text { Prepared } \\
\underline{\text { Model }} \\
\text { (6)Rec }\end{array}$ & $\begin{array}{l}\underline{\text { Socio- }} \\
\underline{\text { economic }} \\
\underline{\text { Model }} \\
(7) \operatorname{Rec}\end{array}$ \\
\hline Electricity & $\begin{array}{l}0.0553^{* * *} \\
(0.012)\end{array}$ & $\begin{array}{l}-0.0353 \\
(0.022)\end{array}$ & $\begin{array}{l}0.0909^{* * *} \\
(0.021)\end{array}$ & $\begin{array}{l}-0.0619^{*} \\
(0.032)\end{array}$ & $\begin{array}{l}-0.0468^{*} \\
(0.026)\end{array}$ & & \\
\hline Water & $\begin{array}{l}0.0352^{*} \\
(0.021)\end{array}$ & $\begin{array}{l}-0.0789^{* *} \\
(0.031)\end{array}$ & $\begin{array}{l}0.0565^{*} \\
(0.034)\end{array}$ & $\begin{array}{l}-0.137^{* * *} \\
(0.051)\end{array}$ & $\begin{array}{l}-0.153^{* * *} \\
(0.057)\end{array}$ & & \\
\hline Gas & $\begin{array}{l}0.0339^{* *} \\
(0.015)\end{array}$ & $\begin{array}{l}-0.051^{* * *} \\
(0.013)\end{array}$ & $\begin{array}{l}0.0551^{* *} \\
(0.024)\end{array}$ & $\begin{array}{l}-0.089^{* * * *} \\
(0.022)\end{array}$ & $\begin{array}{l}-0.0756^{* * *} \\
(0.025)\end{array}$ & & \\
\hline Phone & $\begin{array}{l}0.0106 \\
(0.013)\end{array}$ & $\begin{array}{l}-0.0516^{*} \\
(0.027)\end{array}$ & $\begin{array}{l}0.0176 \\
(0.022)\end{array}$ & $\begin{array}{l}-0.090^{* *} \\
(0.045)\end{array}$ & $\begin{array}{l}-0.0691^{*} \\
(0.041)\end{array}$ & & \\
\hline Trans & $\begin{array}{l}0.0345^{* * *} \\
(0.012)\end{array}$ & $\begin{array}{l}-0.0176 \\
(0.026)\end{array}$ & $\begin{array}{l}0.0576^{* * *} \\
(0.019)\end{array}$ & $\begin{array}{l}-0.0310 \\
(0.042)\end{array}$ & $\begin{array}{l}-0.0309 \\
(0.032)\end{array}$ & & \\
\hline Insurance & $\begin{array}{l}0.317^{* * *} \\
(0.095)\end{array}$ & $\begin{array}{l}0.0943 \\
(0.328)\end{array}$ & $\begin{array}{l}0.519^{* * * *} \\
(0.161)\end{array}$ & $\begin{array}{l}0.158 \\
(0.555)\end{array}$ & & $\begin{array}{l}1.041^{* *} \\
(0.507)\end{array}$ & \\
\hline Protection & $\begin{array}{l}0.601^{* * *} \\
(0.168)\end{array}$ & $\begin{array}{l}0.978^{* * *} \\
(0.359)\end{array}$ & $\begin{array}{l}1.000^{* * *} \\
(0.278)\end{array}$ & $\begin{array}{l}1.684^{* * * *} \\
(0.583)\end{array}$ & & $\begin{array}{l}1.262^{* * * *} \\
(0.427)\end{array}$ & \\
\hline Generator & $\begin{array}{l}0.141 \\
(0.104)\end{array}$ & $\begin{array}{l}0.435^{* *} \\
(0.218)\end{array}$ & $\begin{array}{l}0.242 \\
(0.175)\end{array}$ & $\begin{array}{l}0.752^{* *} \\
(0.377)\end{array}$ & & $\begin{array}{l}0.512 \\
(0.339)\end{array}$ & \\
\hline Irene & $\begin{array}{l}-0.0124 \\
(0.094)\end{array}$ & $\begin{array}{l}0.534^{* *} \\
(0.249)\end{array}$ & $\begin{array}{l}-0.0226 \\
(0.160)\end{array}$ & $\begin{array}{l}0.925^{* *} \\
(0.433)\end{array}$ & & & $\begin{array}{l}0.854^{* *} \\
(0.392)\end{array}$ \\
\hline Age & $\begin{array}{l}0.00728^{* *} \\
(0.004)\end{array}$ & $\begin{array}{l}0.00328 \\
(0.010)\end{array}$ & $\begin{array}{l}0.0128^{* *} \\
(0.006)\end{array}$ & $\begin{array}{l}0.00555 \\
(0.017)\end{array}$ & & & $\begin{array}{l}-0.00654 \\
(0.015)\end{array}$ \\
\hline Education & $\begin{array}{l}0.107^{*} \\
(0.065)\end{array}$ & $\begin{array}{l}0.410^{* *} \\
(0.160)\end{array}$ & $\begin{array}{l}0.197^{*} \\
(0.112)\end{array}$ & $\begin{array}{l}0.708^{* *} \\
(0.278)\end{array}$ & & & $\begin{array}{l}0.617^{* *} \\
(0.253)\end{array}$ \\
\hline Income & $\begin{array}{l}0.00130 \\
(0.012)\end{array}$ & $\begin{array}{l}0.00147 \\
(0.027)\end{array}$ & $\begin{array}{l}0.000847 \\
(0.021)\end{array}$ & $\begin{array}{l}0.00240 \\
(0.047)\end{array}$ & & & $\begin{array}{l}-0.00515 \\
(0.046)\end{array}$ \\
\hline Size & $\begin{array}{l}0.0993^{* *} \\
(0.039)\end{array}$ & $\begin{array}{l}0.0404 \\
(0.100)\end{array}$ & $\begin{array}{l}0.176^{* * *} \\
(0.067)\end{array}$ & $\begin{array}{l}0.0689 \\
(0.173)\end{array}$ & & & $\begin{array}{l}-0.0101 \\
(0.170)\end{array}$ \\
\hline Years & $\begin{array}{l}0.00847^{* *} \\
(0.004)\end{array}$ & $\begin{array}{l}-0.0111 \\
(0.008)\end{array}$ & $\begin{array}{l}0.0142^{* *} \\
(0.006)\end{array}$ & $\begin{array}{l}-0.0194 \\
(0.014)\end{array}$ & & & $\begin{array}{l}0.00615 \\
(0.011)\end{array}$ \\
\hline Wind & $\begin{array}{l}0.0246^{* * *} \\
(0.007)\end{array}$ & & $\begin{array}{l}0.0453^{* * * *} \\
(0.012)\end{array}$ & & & & \\
\hline
\end{tabular}




\begin{tabular}{|c|c|c|c|c|c|c|}
\hline Constant & $\begin{array}{l}-4.083^{* * *} \\
(0.630)\end{array}$ & $\begin{array}{l}-7.217^{* * *} \\
(1.142)\end{array}$ & & & & \\
\hline Cut1_Constant & $\begin{array}{l}0.262 \\
(1.212)\end{array}$ & & $\begin{array}{l}0.396 \\
(1.503)\end{array}$ & $\begin{array}{l}-3.060^{* * * *} \\
(0.386)\end{array}$ & $\begin{array}{l}-0.404 \\
(0.478)\end{array}$ & $\begin{array}{l}0.797 \\
(1.498)\end{array}$ \\
\hline Cut2_Constant & $\begin{array}{l}1.800 \\
(1.209)\end{array}$ & & $\begin{array}{l}3.061^{* *} \\
(1.513)\end{array}$ & $\begin{array}{l}-0.757^{* * *} \\
(0.266)\end{array}$ & $\begin{array}{l}1.530^{* * *} \\
(0.520)\end{array}$ & $\begin{array}{l}2.653^{*} \\
(1.504)\end{array}$ \\
\hline rho & $\begin{array}{l}0.0141 \\
(0.359)\end{array}$ & & & & & \\
\hline pseudo $R^{2}$ & & 0.147 & 0.249 & 0.153 & 0.065 & 0.037 \\
\hline Log-likelihood & -673.5 & -557.2 & -116.0 & -130.9 & -144.4 & -148.8 \\
\hline $\operatorname{LR}\left(\chi^{2}\right)$ Test & 205.1 & 146.2 & 46.72 & 31.96 & 15.01 & 9.228 \\
\hline$N$ & 1042 & 1042 & 150 & 150 & 150 & 150 \\
\hline
\end{tabular}

Note: Standard errors in parentheses ${ }^{*} \mathrm{p}<0.10,{ }^{* *} \mathrm{p}<0.05,{ }^{* * *} \mathrm{p}<0.01$ 
Table 1.4: Marginal Effects for Household Damage and Recovery

\begin{tabular}{|c|c|c|c|c|}
\hline & Damage & $\begin{array}{l}\mathrm{NR} \\
(\mathrm{Rec}=1)\end{array}$ & $\begin{array}{l}\mathrm{AR} \\
(\mathrm{Rec}=2)\end{array}$ & $\begin{array}{l}\text { FR } \\
(\mathrm{Rec}=3)\end{array}$ \\
\hline Electricity & $0.0189^{* * *}$ & $0.00592^{*}$ & $0.00846^{*}$ & $-0.0144^{*}$ \\
\hline Water & $0.0118^{*}$ & $0.0131^{* *}$ & $0.0187^{* *}$ & $-0.0318^{* * *}$ \\
\hline Gas & $0.0115^{* *}$ & $0.00847^{* * *}$ & $0.0121^{* * *}$ & $-0.0206^{* * *}$ \\
\hline Phone & 0.00367 & $0.00858^{*}$ & $0.0122^{*}$ & $-0.0208^{* *}$ \\
\hline Transportation & $0.0120^{* * *}$ & 0.00297 & 0.00424 & -0.00721 \\
\hline Insurance (d) & $0.105^{* * * *}$ & -0.0157 & -0.0205 & 0.0362 \\
\hline Protection $(\mathrm{d})$ & $0.235^{* * *}$ & $-0.117^{* * *}$ & $-0.280^{* *}$ & $0.397^{* * *}$ \\
\hline Generator (d) & 0.0516 & $-0.0655^{*}$ & $-0.113^{*}$ & $0.178^{* *}$ \\
\hline Irene $(d)$ & -0.00472 & $-0.102^{*}$ & $-0.0993^{* * *}$ & $0.202^{* *}$ \\
\hline Age & $0.00266^{* *}$ & -0.000531 & -0.000759 & 0.00129 \\
\hline Education & $0.0411^{*}$ & $-0.0677^{* *}$ & $-0.0967^{* *}$ & $0.164^{* *}$ \\
\hline Income & 0.000176 & -0.000230 & -0.000329 & 0.000559 \\
\hline Size & $0.0367^{* * * *}$ & -0.00659 & -0.00941 & 0.0160 \\
\hline Years & $0.00295^{* *}$ & 0.00185 & 0.00265 & -0.00450 \\
\hline Wind & $0.00945^{* * *}$ & & & \\
\hline $\mathrm{N}$ & 1042 & 150 & 150 & 150 \\
\hline
\end{tabular}

Notes: NR is the level of "Not Recovered", AR is the level of "Almost Recovered" and FR is the level of "Fully Recovered". (d) is for discrete change of dummy variable from 0 to $1{ }^{*} \mathrm{p}<0.10,{ }^{* *} \mathrm{p}<0.05,{ }^{* * *} \mathrm{p}<0.01$ 
Table 1.5: Predicted Probabilities on Recovery by Utility Disruption

\begin{tabular}{|c|c|c|c|c|}
\hline Variable & Mean & Std. Dev. & Min & Max \\
\hline \multicolumn{5}{|c|}{ Predicted possibilities for Recovery=1 (NR) } \\
\hline Electricity & 0.423 & 0.273 & 0.069 & 0.849 \\
\hline Water & 0.731 & 0.336 & 0.086 & 0.999 \\
\hline Gas & 0.602 & 0.330 & 0.086 & 0.979 \\
\hline Phone & 0.585 & 0.336 & 0.074 & 0.977 \\
\hline \multicolumn{5}{|c|}{ Predicted possibilities for Recovery=2 (AR) } \\
\hline Electricity & 0.420 & 0.152 & 0.139 & 0.582 \\
\hline Water & 0.200 & 0.230 & 0.001 & 0.571 \\
\hline Gas & 0.300 & 0.220 & 0.020 & 0.580 \\
\hline Phone & 0.306 & 0.217 & 0.021 & 0.580 \\
\hline \multicolumn{5}{|c|}{ Predicted possibilities for Recovery $=3(\mathrm{FR})$} \\
\hline Electricity & 0.157 & 0.154 & 0.012 & 0.485 \\
\hline Water & 0.069 & 0.125 & 0.000 & 0.425 \\
\hline Gas & 0.098 & 0.132 & 0.002 & 0.425 \\
\hline Phone & 0.109 & 0.145 & 0.002 & 0.464 \\
\hline
\end{tabular}

Notes: NR is the level of "Not Recovered", AR is the level of "Almost Recovered" and FR is the level of "Fully Recovered". 
Table 1.6: Predicted Probabilities on Recovery by Irene and Education

\begin{tabular}{llll}
\hline Irene=0 & $\mathrm{NR}$ & $\mathrm{AR}$ & $\mathrm{FR}$ \\
\hline Less than high school & 0.571 & 0.380 & 0.050 \\
High school & 0.400 & 0.508 & 0.096 \\
Some college & 0.244 & 0.579 & 0.177 \\
Bachelor's degree or higher & 0.137 & 0.558 & 0.305 \\
& & & \\
\hline Irene=1 & $\mathrm{NR}$ & $\mathrm{AR}$ & $\mathrm{FR}$ \\
\hline Less than high school & 0.345 & 0.538 & 0.117 \\
High school & 0.206 & 0.583 & 0.211 \\
Some college & 0.114 & 0.534 & 0.352 \\
Bachelor's degree or higher & 0.059 & 0.416 & 0.525
\end{tabular}

Change from Irene $=0$ to Irene $=1$

\begin{tabular}{llll} 
& NR & AR & FR \\
\hline Less than high school & -0.226 & 0.158 & 0.067 \\
High school & -0.194 & 0.075 & 0.115 \\
Some college & -0.130 & -0.045 & 0.175 \\
Bachelor's degree or higher & -0.078 & -0.142 & 0.220
\end{tabular}

Notes: NR is the level of "Not Recovered", AR is the level of "Almost Recovered" and FR is the level of "Fully Recovered". Irene=1 implies that respondents had Irene experience and Irene $=0$ for otherwise. 


\section{Figures}

Figure 1.1: The Disaster Resilience Triangle

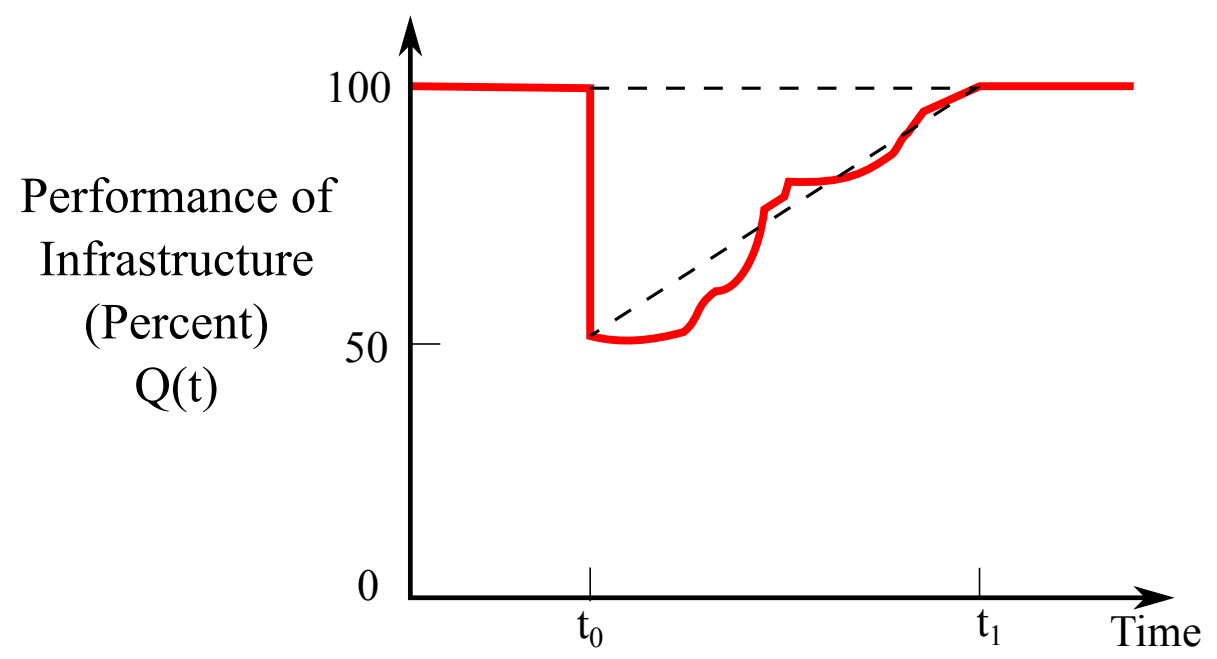

Notes: The figure depicts the idea that resilience depends on the extent of damages due to utility disruptions and the time needed to recover (adopted from Bruneau et al. (2003) in Zobel (2011)). 
Figure 1.2: The Decision Framework

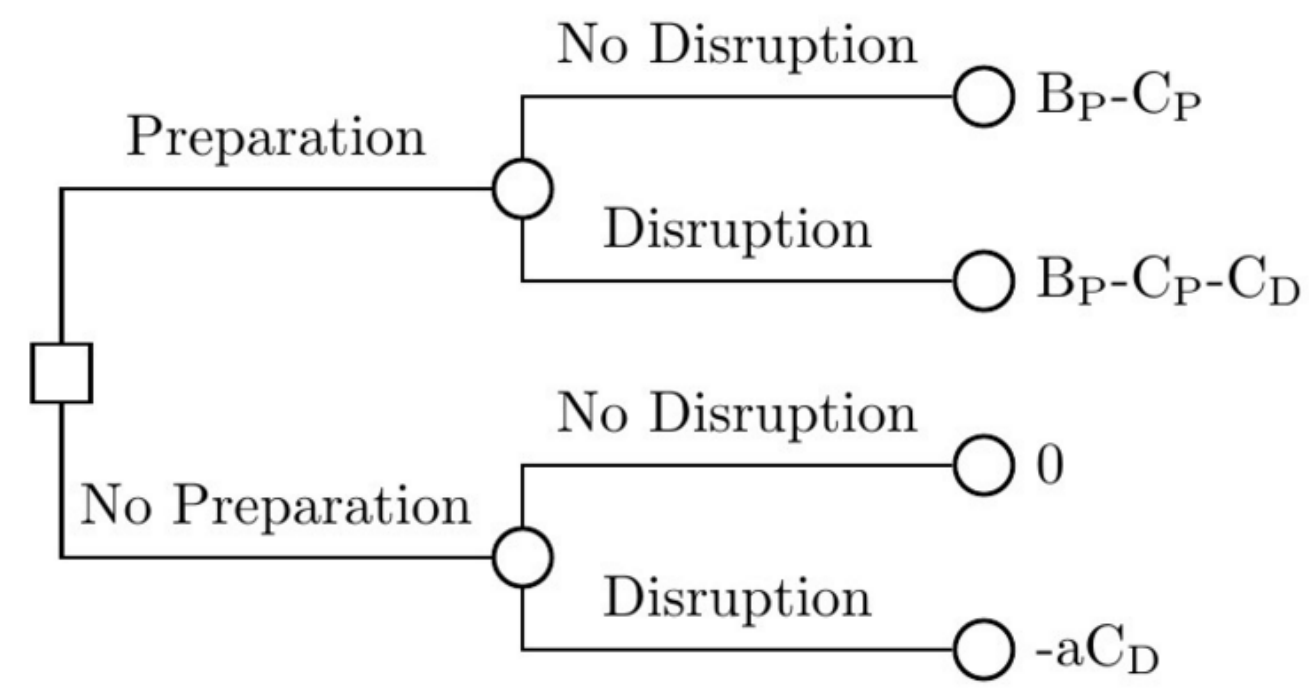

Notes: This figure shows the decision framework of preparedness measures undertaken by households and the associated outcomes. The net benefit of hurricane preparedness is measured by the benefit of preparation minus the cost $\left(B_{P}-C_{P}\right)$. If households were prepared but incurred damages, the outcome becomes $B_{P}-C_{P}-C_{D}$, where $C_{D}$ presents the cost of disruptions. Note that the cost of disruptions tends to be worse for unprepared households, captured by a weight $a$, where $a>1$. 
Figure 1.3: Dynamic Framework of Household Resilience

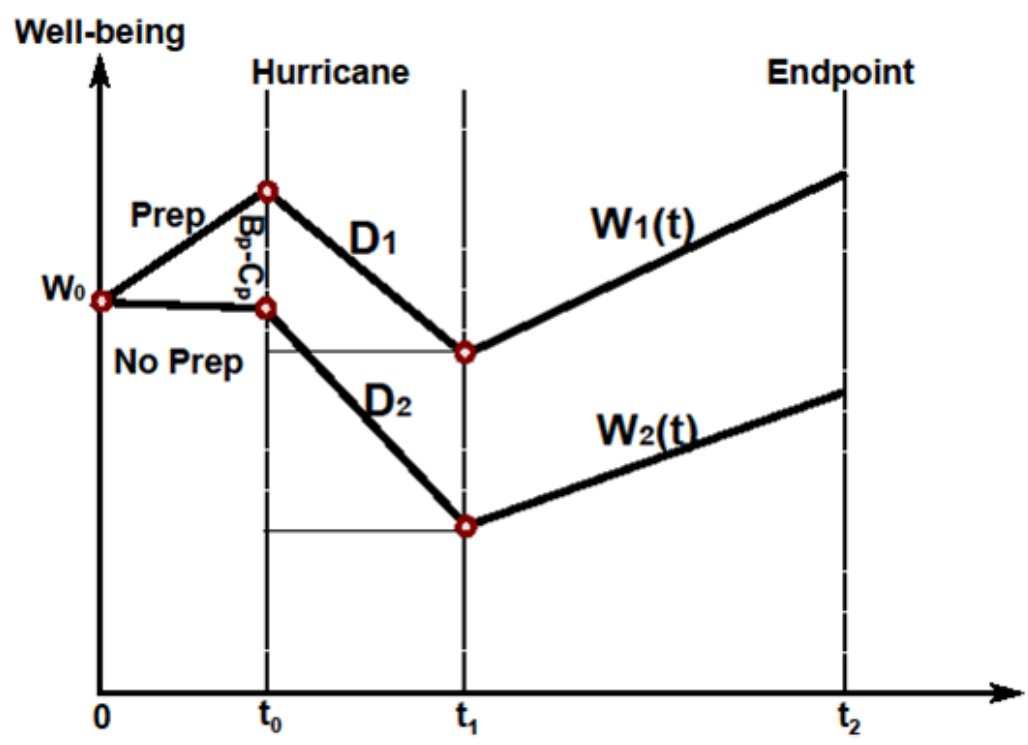

Figure 1.3a $B P>C_{P}$

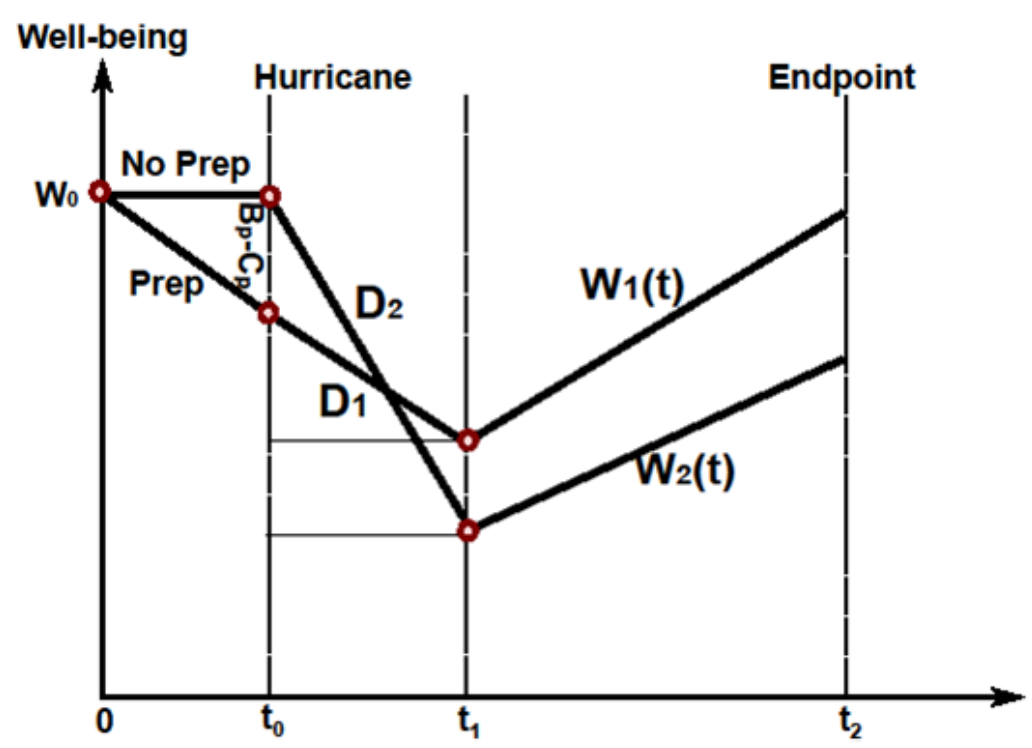

Figure $1.3 \mathrm{~b} B P<C_{P}$

Notes: This figure illustrates the temporal dynamics of household responses to the consequences of hurricane and the recovery process. Figure $1.3 \mathrm{a}$ is the case when the benefit of preparedness is greater than the cost. Figure $1.3 \mathrm{~b}$ is the case when the benefit of preparedness is lower than the cost. Household resilience $R_{i}$ can be measured by integrating the areas under the $W_{i}(t)$ curves. 
Figure 1.4: Survey Questions on Damage and Recovery

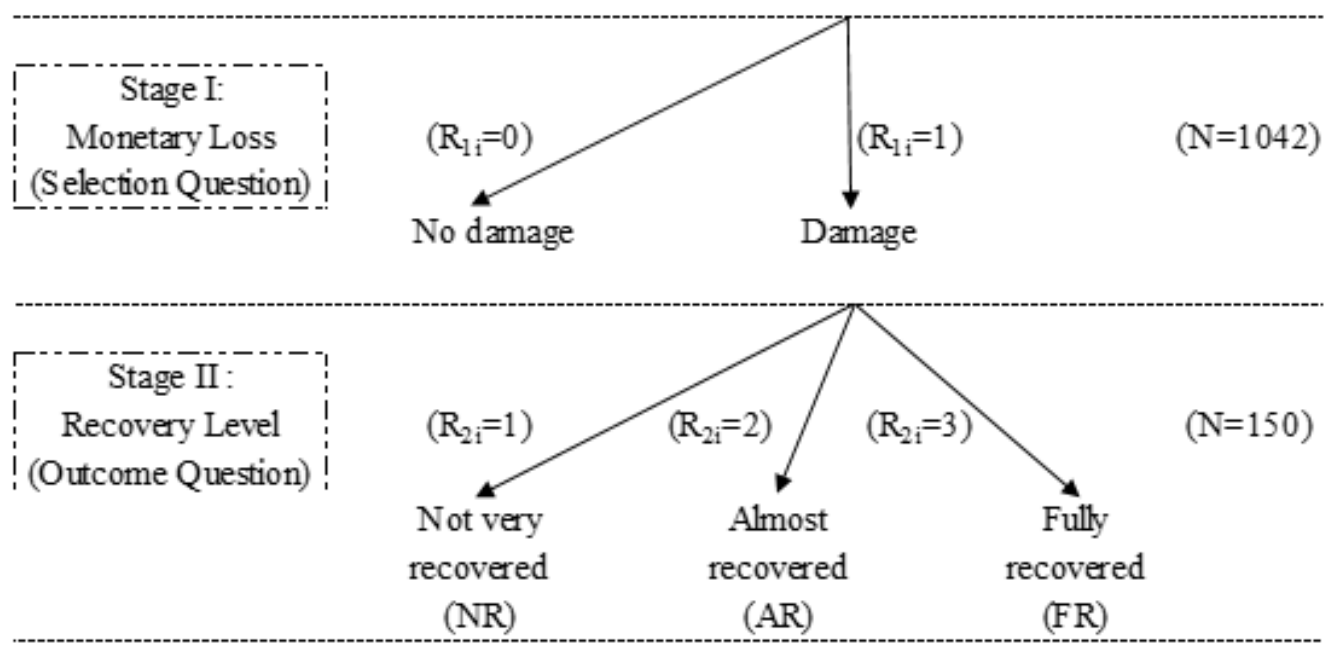

Notes: The survey was conducted eight months after Hurricane Sandy (2012).

Households were first asked to report their monetary losses, and those who reported a positive amount of loss were then asked to rate their recovery levels. 
Figure 1.5: Location of Surveyed Respondents by State

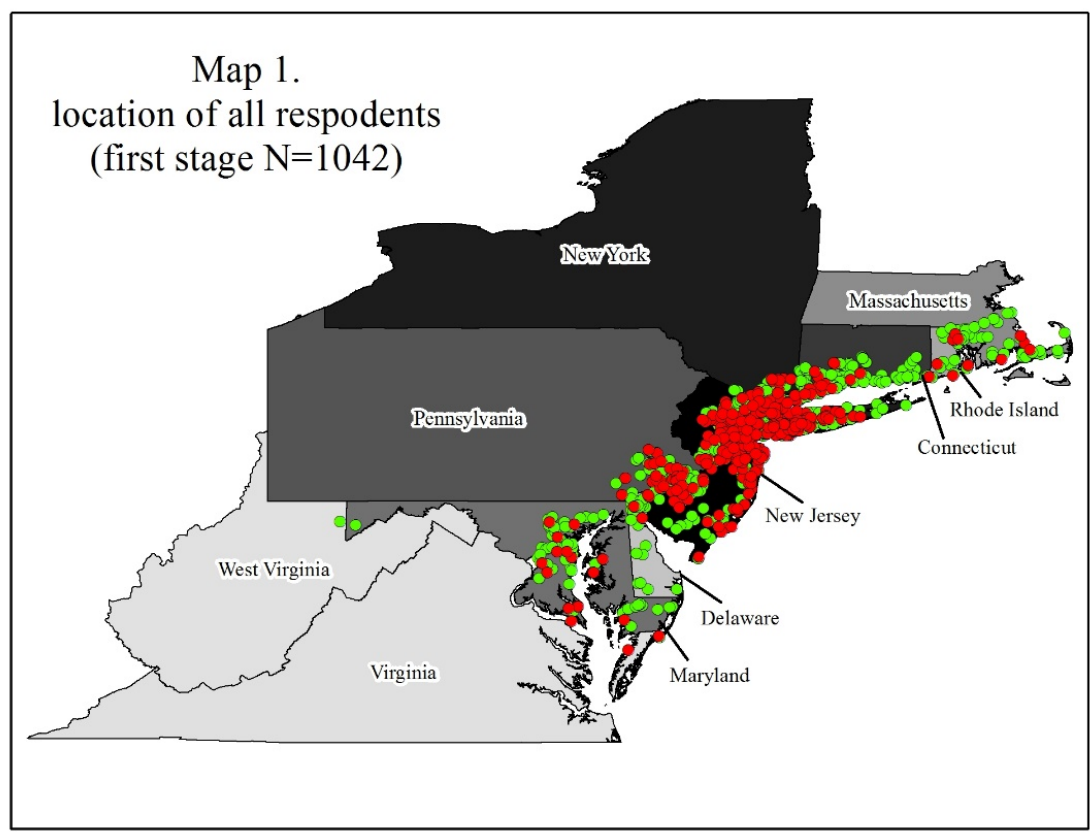

\section{Legend}

Number of respondents

\begin{tabular}{|l|l}
\hline & $381(\mathrm{NJ})$ \\
\hline & $296(\mathrm{NY})$ \\
\hline & $126(\mathrm{CT})$ \\
\hline & $76(\mathrm{PA})$ \\
\hline & $69(\mathrm{MD})$ \\
\hline & 33 (MA) \\
\hline & 27 (RI) \\
\hline & 26 (DE) \\
\hline & 3 (VA) \\
\hline & 1 (WV) \\
\hline$\quad \begin{array}{l}\text { Respondents } \\
\text { (Damage }=1)\end{array}$ \\
Respondents \\
(Damage=0)
\end{tabular}

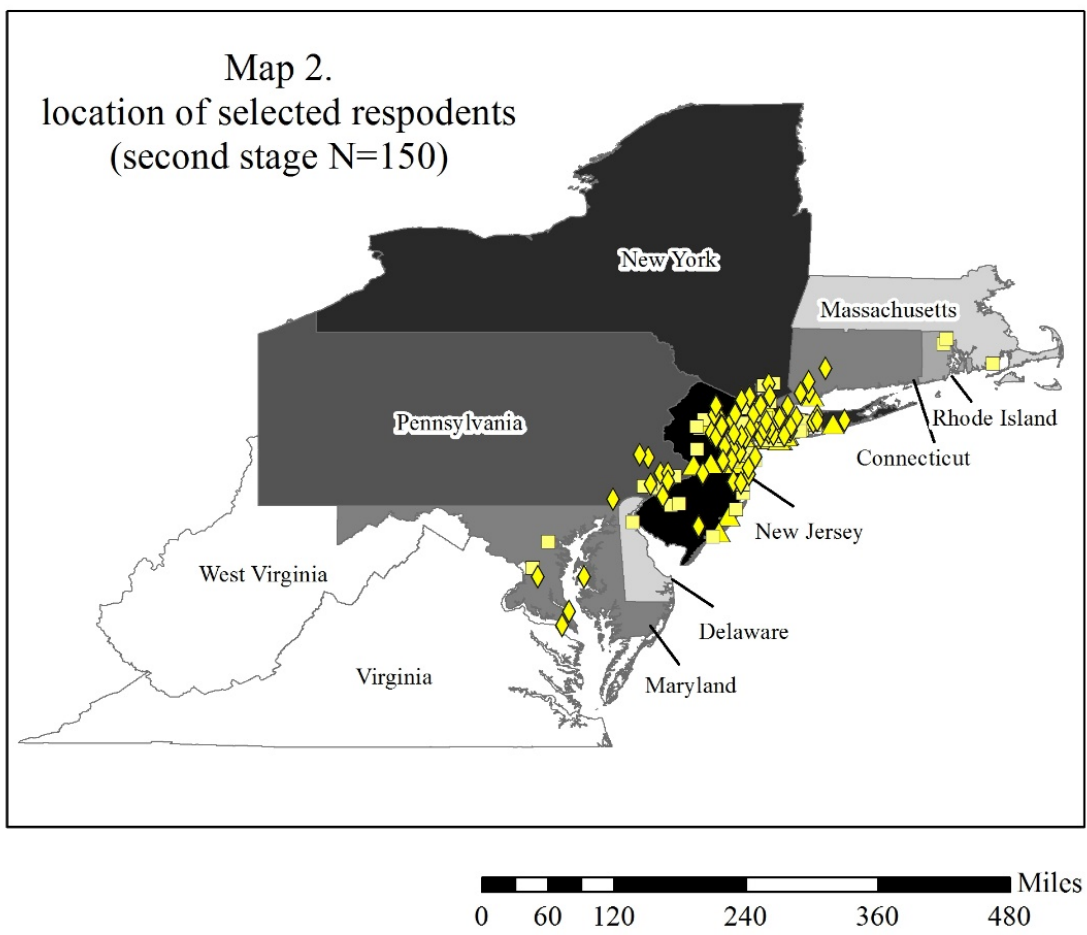

\section{Legend}

Number of respondents

\begin{tabular}{|c|c|}
\hline & $64(\mathrm{NJ})$ \\
\hline & $57(\mathrm{NY})$ \\
\hline & $11(\mathrm{PA})$ \\
\hline & $6(\mathrm{CT} \& \mathrm{MD})$ \\
\hline & $2(\mathrm{RI})$ \\
\hline & 1 (DE \& MA) \\
\hline & 0 (VA \& WA) \\
\hline$\diamond$ & FR respondents \\
\hline$\square$ & AR respondents \\
\hline$\triangle$ & NR respondents \\
\hline
\end{tabular}

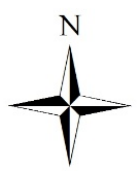


Figure 1.6: Statistics on Utility Disruptions

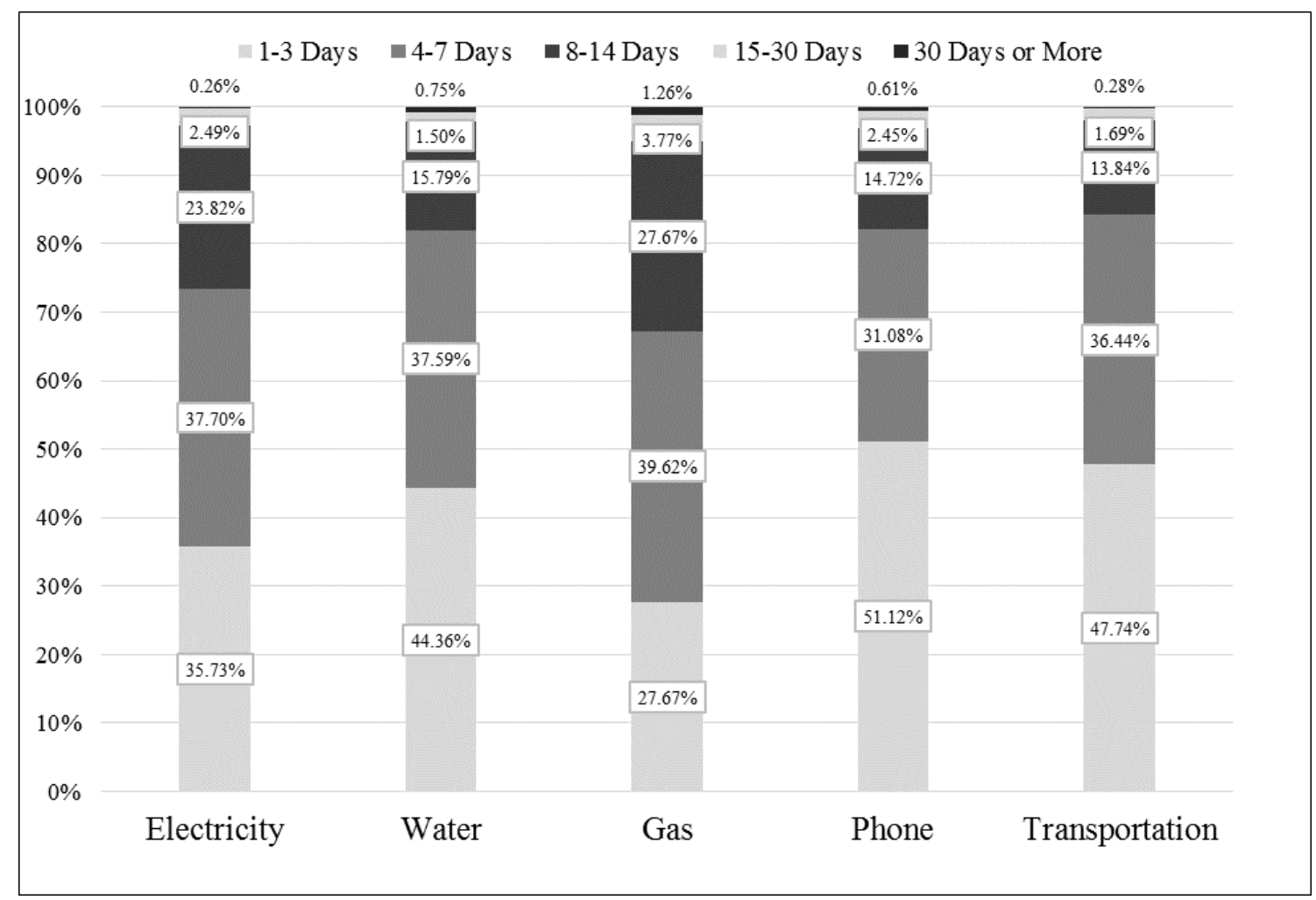

Notes: Respondents were asked to report how many days they experienced disruptions on five different types of utility services. This figure shows the percentage and duration of each utility disruption reported. 
Figure 1.7: Predicted Probabilities on Damage by Wind Speed

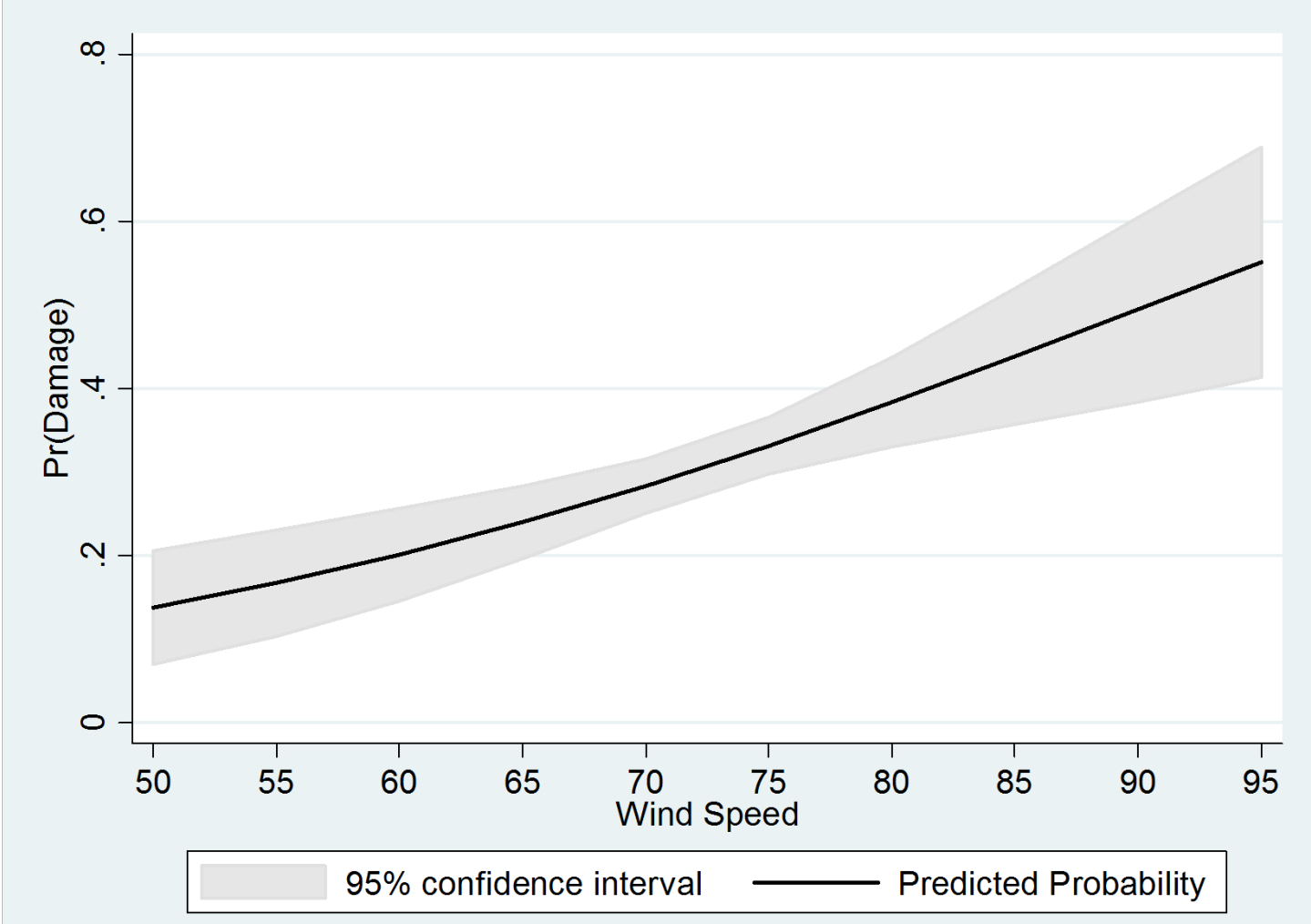

Notes: The figure presents the predicted probabilities of reporting positive damages by households exposed to different wind speeds and the $95 \%$ confidence intervals around the predictions. The probability is ranged from 0.14 when wind speed is 50 miles per hour to 0.55 when wind speed is 95 miles per hour. 
Figure 1.8: Predicted Probabilities on Recovery by Utility Disruption
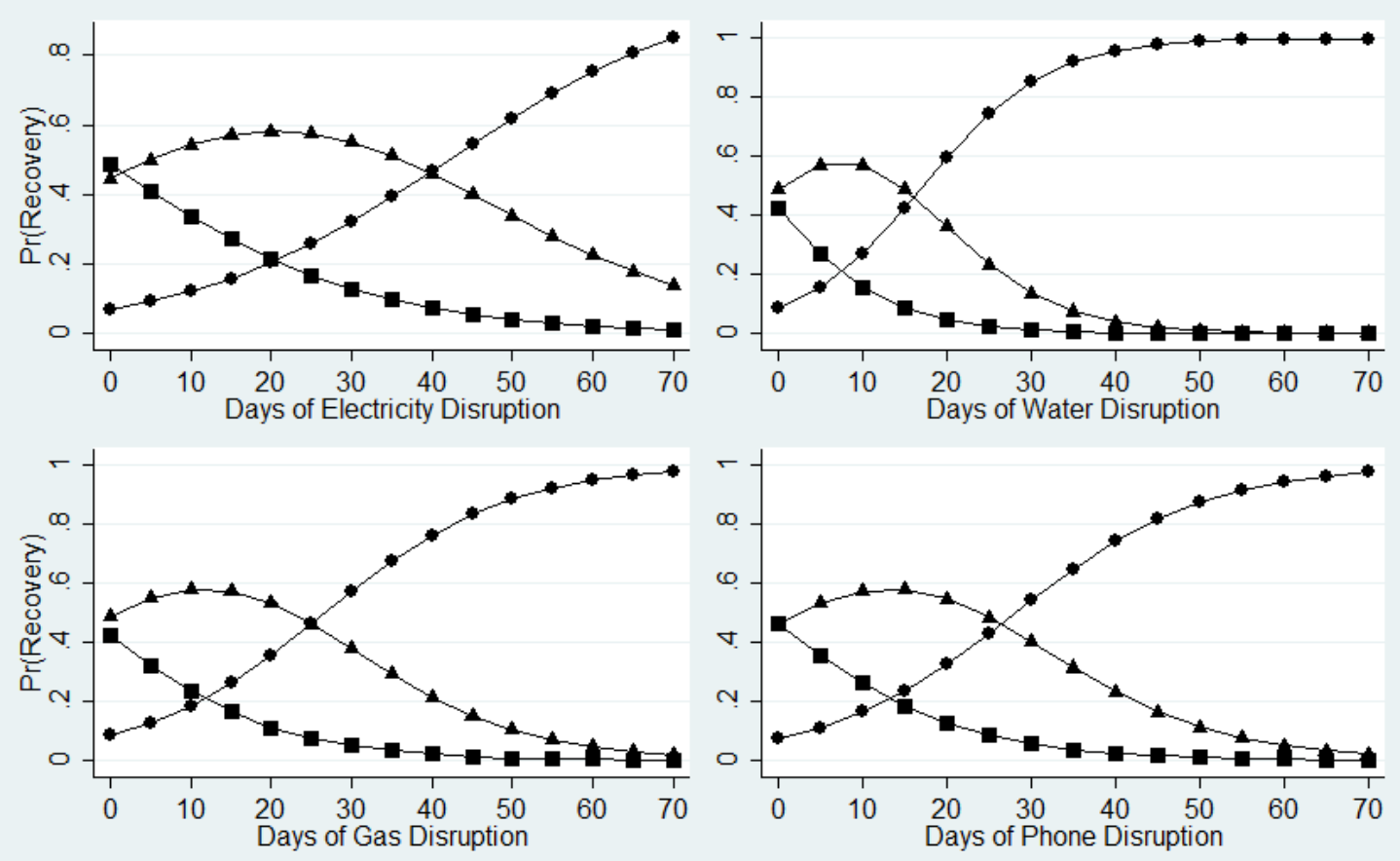

$\longrightarrow$ Not Well Recovered

$\_$Almost Recovered

Fully Recovered 
Figure 1.9: The Role of Installing Window Protection on Recovery

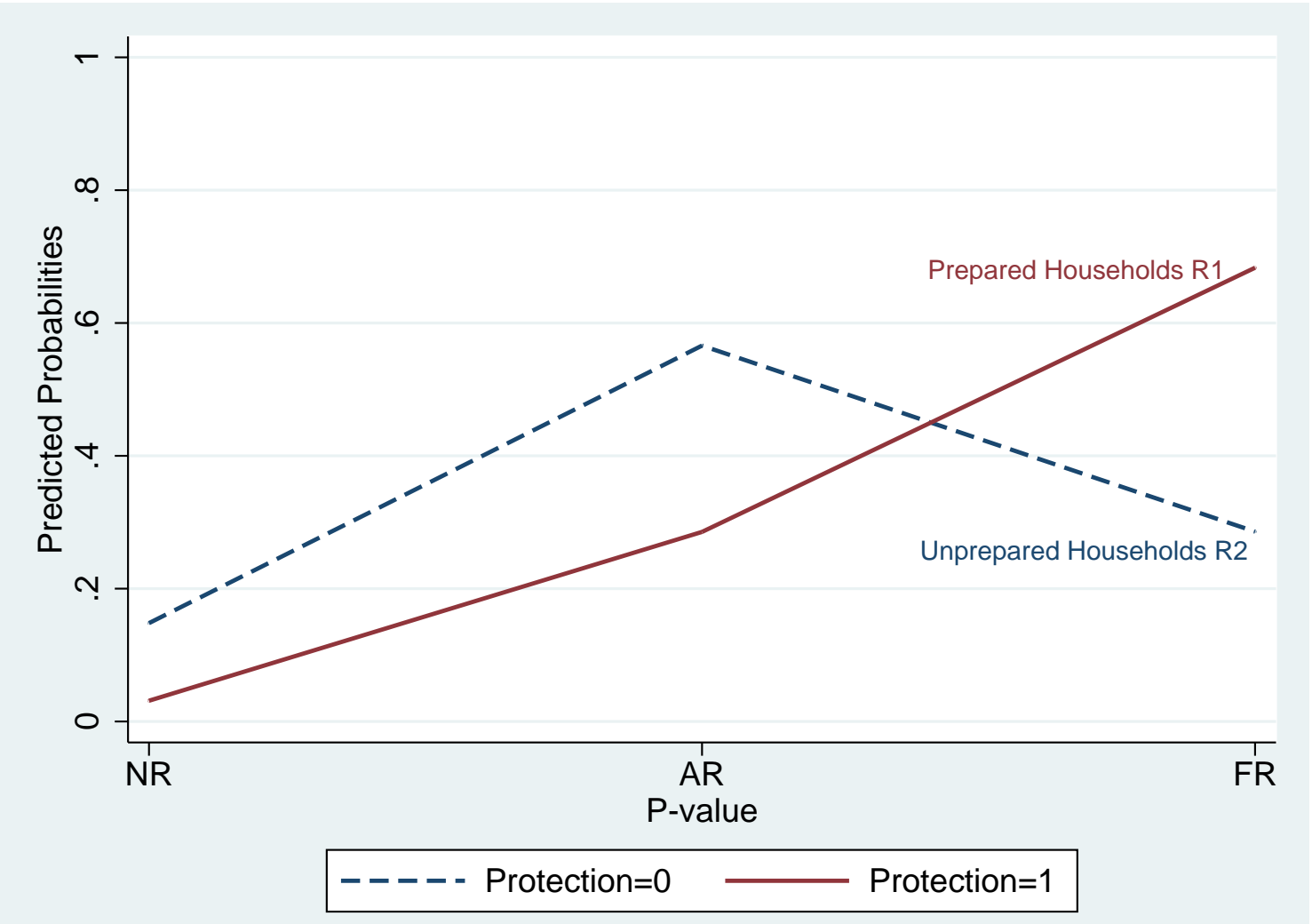

Notes: This figure presents the predicted probabilities on household recovery levels with and without wind resistant windows. NR is the level of "Not Recovered", AR is the level of "Almost Recovered", and FR is the level of "Fully Recovered". 
Figure 1.10: The Role of Owning a Generator on Recovery

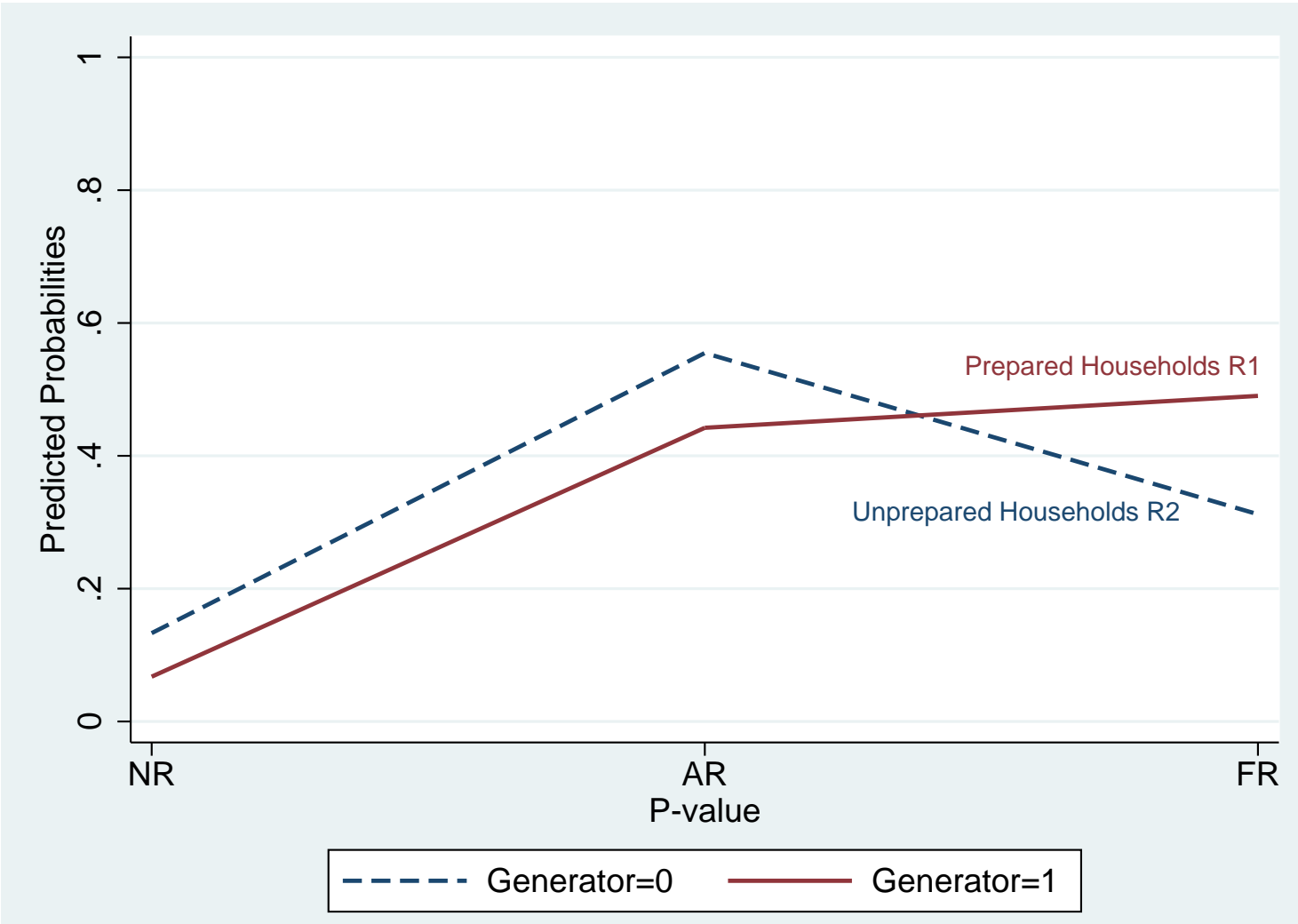

Notes: This figure presents the predicted probabilities on household recovery levels with and without a generator. NR is the level of "Not Recovered", AR is the level of "Almost Recovered", and FR is the level of "Fully Recovered". 
CHAPTER 2

\section{SPATIAL HETEROGENEITY AND HOUSEHOLD PREFERENCES FOR SEA LEVEL RISE ADAPTATION PLAN IN FLORIDA}

\section{$2.1 \quad$ Introduction}

Accumulating evidence indicates that global sea levels have been rising at an accelerating rate, expecting to have a pronounced impact all over the world in the coming century. This trend, linked with global warming, is posing a great risk to communities living in the low elevation coastal areas. Sea level rise (SLR) also increases the frequency of various disasters such as tidal inundations, flooding, storm surges, salt water intrusions, and other related hazards. Ecological environments along coasts could also experience changes, for example, a large number of inhabitants may be forced to move inland, and cities, industry, and agriculture may be severely and adversely affected (Wang, Chen, Zhang, \& Shen, 1995). The risk of rising seas will continue to increase due to the fast growing population in coastal cities and tourism, and damage cost estimations are substantial (Bates et al., 2008).

The rate of rise in sea levels is not spatially uniform so the effects on coastal areas can be considerably different among regions (Intergovernmental Panel on Climate Change, 2007). Florida is particularly vulnerable to the effects of SLR due to its low topography, porous geology, subtropical climate, and densely populated coastal counties. According to Florida Oceans and Coastal Council (2010), Florida has more than 1,200 miles of coastline, almost 4,500 square miles of estuaries and bays, and more than 6,700 square miles of other coastal waters. The entire state lies within the Atlantic Coastal Plain, with a maximum elevation less than 400 feet above sea level, and most of Florida's residents live less than 60 miles from the Atlantic Ocean or the Gulf of Mexico. Furthermore, Miami and the Tampa-St. Petersburg regions have 
been identified as two of the ten most vulnerable cities to the threats from SLR in the world, along with Amsterdam, Rotterdam, Osaka-Kobe, Tokyo, Nagoya, greater New York, New Orleans, and Virginia Beach (Hanson et al., 2010).

Using LiDAR map, Harlem (2008) showed that there will be only $83 \%$ of land surface remaining in South Florida by 2040 and $62 \%$ by 2100 . He also predicted that $97 \%$ of South Florida will be underwater by 2159 . Unfortunately, such a problem is not only present in South Florida - even a seemingly small rise in sea level can have a dramatic impact on many of the coastal communities statewide. Figure 2.1 shows the areas of Florida that would be inundated at various stages of SLR from year 2030 to 2100 . The maps clearly depict that a rise in sea level over time would inundate and flood thousands of acres of highly developed coastal communities along Florida's Atlantic Coast, the Florida Gulf Coast, and the Florida Keys. Significant flooding and environmental changes can also be observed in the Everglades. Therefore, adapting to the climatic change induced sea level rise will be crucial for Florida's vulnerable communities.

There are usually three stages to building resilience to SLR in many coastal communities or counties across the United States: 1) becoming aware that sea level rise may be a local threat; 2) assessing main vulnerabilities using risk-based analysis; and 3) developing adaptation plans and policies to address priority vulnerabilities and reduce major risks over the short terms and long terms (Lausche \& Maier, 2013). The practical task for low-lying communities is to reduce the sea's attacks upon land, buildings and water supplies, which can be done by conserving natural sea defenses like beaches, mangroves and coral reefs, and by preserving water resources by water conservation programs (Ince, 1990). The transportation systems and land resources, among other vulnerable infrastructures, must also be integrated into climate adaptation plans to address the impacts of rising seas. Without a doubt, 
appropriate and specific adaptation plans are essential in Florida in preparing for SLR, which in turn makes local coasts and communities more resilient. However, a major information gap exists in understanding the associated risks and how to proceed with adaptation initiatives at the local level.

The main objective of this study is to analyze public perceptions and preferences on various sea level rise adaptation plans in Florida. Specifically, a series of choice experiments embedded in a household survey of Florida's selected communities are used to: 1) examine the determinants of households' preferences for short term adaption plans and long term adaptation plans; 2) identify the spatially heterogeneous preferences in household choices, by incorporating detailed spatial information generated by Geographical Information Systems (GIS) into the survey data; 3) investigate the differences and similarities in perceptions and preferences among Florida's yearlong and seasonal residents. Warm weather during the winter season, coastal amenities, and absence of state income tax largely contribute to attracting many people to come and stay for a few months of the year in Florida. Many of these residents own properties in Florida though they tend to avoid staying here during hurricane seasons. This type of residents may perceive risks imposed by coastal hazards differently compared to yearlong residents (Mozumder et al., 2014). Against this backdrop, this study pays special attention on seasonal residents' preferences toward sea level rise adaptation plans.

The rest of the study is organized as follows. Section 2.2 provides the climate adaptation literature that relates to public preferences for preventing climate change induced impacts. Section 2.3 introduces the survey and detailed descriptions of the choice experiment design. Section 2.4 presents the sample characteristics of the survey data and GIS data. Section 2.5 explains the choice experiment method and hypotheses tested in the study. The results of the estimated choice models are 
presented in Section 2.6. Section 2.7 discusses the willingness to pay and economic values on different attributes of SLR adaptation plans and Section 2.8 concludes the study with some policy implications.

\subsection{Spatial Heterogeneity and Preferences for Adaptation}

\subsubsection{Previous Research on Climate Change Adaptation}

Empirical studies related to public preferences regarding climate adaptation practices using non-market valuation are available but limited. Berk and Fovell (1999) presented one of the first studies to investigate the effects of climate change. The respondents from Los Angeles in their survey were presented with eight different climate change scenarios and their willingness to pay was found to be influenced by their concerns regarding quality of life, wildlife habitats, the economy, and what future generations will inherit.

Viscusi and Zeckhauser (2006) assessed the risk perceptions of climate change and valued climate change mitigation policies. Harvard University graduate students were asked to express their attitude on a more aggressive or less aggressive policy action if the likely consequences of climate change become more uncertain. The slight majority $(51 \%)$ believed that the policy should be more aggressive and they were

willing to pay more to reduce climate change risks. Lee and Cameron (2008) also addressed the question to examine U.S. residents' perceptions and found that people were generally more willing to pay for climate change mitigation if they believe that the impacts will be substantially, instead of moderately, harmful. Brouwer and Schaafsma (2012) investigated public perception and valuation of alternative flood risk mitigation and adaptation strategies using choice experiments. Their findings 
showed that respondents' willingness to pay for flood insurance depends on where people live (along the coast or the river), their risk perceptions, and income levels.

A number of other scholars have focused more closely on the impacts of sea level rise and adaptation policies. Using revealed preference travel cost method, Whitehead et al. (2009) estimated the economic effects of SLR on marine recreational shore fishing in North Carolina. They found that the welfare losses are potentially substantial, ranging up to a present value of about $\$ 1.3$ billion over 75 years, using conservative estimates of fishing participation growth and a $2 \%$ discount rate.

Discrete choice experiments have been widely employed in the wake of SLR preparation. Kloos and Baumert (2014) studied the preferences of vulnerable people in response to SLR in Alexandria, Egypt. A choice experiment was conducted to assess the willingness to participate in voluntary resettlement programs. Results showed that for those who were willing to resettle, factors such as transparency of the relocation process, public/social infrastructure, financial compensation, housing and income/job security can significantly influence their choices. However, a large share of the population was not willing to resettle given the proposed alternative resettlement programs included in their choice experiment. Birol, Koundouri, and Kountouriset (2009) investigated the flood risk reduction policies due to global warming and increasing sea levels in the Upper Silesia region of Poland. Local residents in their study were found to be willing to accept an increase in local taxation to reduce flood risks. Remoundou et al. (2015) elicited willingness to pay for mitigation measures against specific natural hazard concerns caused by sea level rise and rise in sea temperature in Santander, a coastal region in Northern Spain. Results suggested that people valued positively benefits in terms of increased biodiversity and recreation opportunities, as well as health risk reduction. 


\subsubsection{Distance and Spatial Heterogeneity}

Spatial factors influence and shape public preferences and willingness to pay for changes in ecosystem service provision at study sites (Schaafsma, Brouwer, \& Rose, 2012). Brouwer, Martin-Ortega, and Berbel (2010) referred to spatial heterogeneity as the concept that respondents are expected to value changes in environmental good provision differently depending on their place of residence. Johnston, Swallow, and Bauer (2002) observed that stated preference studies rarely incorporate spatial attributes or address spatial patterns in associated econometrics. Studies that ignore the spatial heterogeneity could also produce biased parameter estimates even with a representative sample (Concu, 2007).

Some studies have considered spatial preference heterogeneity by estimating separate choice models for different locations or adding regional dummy variables in the choice models. Bergmann, Colombo, and Hanley (2008) investigated heterogeneity in preferences from the development of renewable energy projects in rural areas of Scotland, focusing in particular on any differences between the urban and rural residents. The separated urban and rural models indicated that preferences differed between these two groups. Brouwer et al. (2010) assessed preference heterogeneity related to the spatial distribution of water quality improvements throughout a river basin in Spain. The results indicated that respondents had preferences for the improvements to acceptable levels throughout the entire river basin but were not willing to pay extra to reach a more than good condition elsewhere other than their own subbasin.

According to Concu (2007), distance is often considered an important source of preference heterogeneity for three reasons: First, it works as a substitute for the price mechanism, lowering the demand for environmental goods through the purchase of private goods necessary to travel (Scotchmer \& Thisse, 1999). Second, 
it affects the availability of information, in which preferences are dependent on to a certain degree (Beckmann, 1999). Third, the number of substitution opportunities increases as distance decays (Stouffer, 1940).

With the development of GIS software, scholars are able to obtain rich datasets containing distance information or other spatial patterns on surveyed respondents, making it possible to investigate spatial heterogeneity. For example, Jørgensen et al. (2013) incorporated GIS data to study the demand for restoring the Odense River in Denmark, and they found a clear distance-decay effect between both the user and non-user groups. They also found that the demand among non-users was more sensitive to the distance to the resource as well as to potential substitutes. Tait et al. (2012) combined choice experiment and spatially-related water quality data via GIS to evaluate the influence of local water quality on preferences for

river and stream conservation programs in New Zealand. Results showed that those respondents who lived in the vicinity of low quality waterways were willing to pay more for improvements relative to those who lived near high quality waterways.

\subsubsection{Preferences in the Short and Long Term}

Another important issue in climate change adaptation research is the extent to which the timing of the ecosystem impact occurs (Layton \& Brown, 2000). The effects of climatic change will not remain steady as time elapses, but may accelerate, causing more damages to the coastal communities. Therefore, it is necessary to distinguish between potential adaptation strategies that may work in the short and the long term.

Layton and Brown (2000) examined the structure of preferences for mitigating impacts of global climate change that will not occur during the lifetimes of most who 
are alive today. They utilized two time horizon, a near-term impact of 60 years and a longer-term impact of 150 years. The results indicated that people were willing to pay more for greater losses as the authors expected. However, they could not find differences in preferences for the two vastly different time horizons. One possible explanation provided was that the models were not powerful enough to reject the null hypothesis of the same preference. Another explanation was that respondents

just missed or ignored the time attribute, which the authors believed to be unlikely, given their carefully designed and pre-tested survey.

\subsubsection{Preferences among Yearlong and Seasonal Residents}

While Florida is at the forefront of rising coastal vulnerability, it also has some unique features regarding the socio-demographic composition of its residents. In order to effectively assess residents' perceptions of coastal vulnerability and their corresponding preferences, the influence of place attachment should be taken into consideration because it is an emotional bond that affects behavior (Burley, 2010; Snider et al., 2011).

Living in coastal Florida provides a wide variety of benefits to all of its residents. Yearlong residents are able to take advantage of the coastal resources, and seasonal residents can escape the treacherous winter weather and enjoy a temperate climate. Regardless of the reasoning behind being a seasonal or yearlong resident, studies have shown significant differences in place attachment between the two groups. Variations in the level of place attachment correspond to differences seen in attitudes regarding land use, economic development, environmental quality, and civic engagement - all of which influence risk mitigation preferences (Mozumder et al., 2014). 
In a study comparing the attitudes of land use controls and economic development activities between seasonal and recreational homeowners, Green et al. (1996) concluded that short-term residents did not connect with the permanent residents in their community because of the lack of local dependence and sense of community. Seasonal residents may not have strong place dependence to their surrounding environment because of the transient nature of their residency.

However, the influence of place attachment on preferences may be outweighed by other socio-economic characteristics, such as income levels. For example, Green et al. (1996) stated that seasonal residents are more willing to pay taxes. Landry et al. (2011) used choice experiment with a stratified sample to investigate public preferences for rebuilding New Orleans' man-made storm defenses, restoring natural storm protection, and improving evacuation options. They targeted residents of the New Orleans metropolitan area as well as other U.S. citizens. The results indicated that individuals were willing to pay for increased storm protection for New Orleans, but other U.S. residents, who were believed to have no place attachment, were willing to pay more than New Orleans residents due to their higher income levels.

\subsection{Survey and Choice Experiment Design}

Our data comes from an internet-based survey conducted by GfK Group. GfK, as one of the world's largest market research organizations, conducts a vast variety of public opinion surveys with its panel sample selection methodology (KnowledgePanel). With the advanced statistical control applied on the sample selection, GfK ensures the representativeness of KnowledgePanel survey samples. In this sur-

vey, we targeted two populations of English-language, survey-taking adults (18 and older) in Florida's selected coastal communities: yearlong residents and seasonal 
residents. The sample, which consisted of either yearlong or seasonal residents, was selected from KnowledgePanel. Additionally, an oversample of only seasonal residents was selected from a non-probability, opt-in web panel. The survey consisted of two stages: an initial screening to determine if the respondent was a yearlong or seasonal resident and the main survey with the study-eligible respondents. The main survey had three primary sections, and participants completed the questions in about 23 minutes (median response time).

The first part of the survey focused on respondents' risk perceptions and concerns on the impacts of sea level rise. Before beginning the survey questions, the respondents were informed that accelerating sea level rise is one of the many effects of global warming, and Florida is particularly vulnerable to the effects due to its low topography, porous geology, subtropical climate, and densely populated coastal counties. Moreover, increased sea levels can have numerous detrimental effects on coastal cities depending on local factors and the rate of rise. Next, the respondents were asked to indicate to what extent they agree or disagree $(=1$ if strongly disagree, 5 if strongly agree) with the following three statements: 1) increased sea level rise is real and we will experience impacts in the short-term future (within 10 - 20 years); 2) increased sea level rise is real and we will experience impacts in the long-term future (within 30 - 50 years); 3) increased sea level rise is not real and there will be no impact. The respondents were also asked to rate their levels of concern $(=1$ if no concern, 4 if high concern) about the projected impacts of SLR on their well-being (health, finances, and property).

The second part of the survey introduced the choice experiment. Respondents were given options for adaptation plans that contained components with varying levels of adaptation strategies and then asked to select which one they would be most willing to pay for. In line with the previous literature, it is necessary to 
take the timing of the ecosystem impact into consideration and compare how and if preferences are sensitive to different time horizons. In Florida, one of the most widely cited SLR estimates is based on a study by the U.S. Army Corps of Engineers (USACE, 2009), which projects a rise of three to seven inches by 2030 and nine to twenty-four inches by 2060 (see Figure 2.2). Based on this estimated projection, we utilized two time horizons: a short-term impact of 10 - 20 years and a long-term impact of 30 - 50 years. As a result, two forms of choice cards, each with separate attributes, were designed in order to accommodate this temporal consideration.

In the case of the short-term adaption plan, respondents were first reminded that the issue of SLR is beyond the control of local communities but they can prepare for the impact through implementing various adaptation strategies. The choice card contained adaptation strategies that have either a short lifespan in terms of the protection effectiveness or represent strategies that are currently under consideration for the span of the next 10 - 20 years. The experimental design consisted of 32 choice tasks, and each respondent was randomly assigned to see one choice card. In the case of the long-term adaption plan, respondents were informed that as global average temperature continues to rise, sea level rise will continue to accelerate, and its effects will be felt in the long term (30 - 50 years). As such, the adaptation strategies that worked in the short term may not be feasible or wise solutions in dealing with the issues in the long term. The choice card represented approaches that favor restructuring of current infrastructure and more holistic approaches to adapting to the accelerating impacts of SLR. The experimental design consisted of 16 choice tasks, and each respondent was randomly shown one choice card. The design in this case was slightly smaller since the number of attributes was lower.

In addition to designing the choice experiment around preferences for the short and long term, we also assessed adaptation strategy finance preferences among 
respondents. After the differences between short-term and long-term adaptation strategies were separated into 2 choice cards, respondents were randomly assigned to have an adaptation plan with matching fund from federal government, i.e. for every $\$ 1$ raised for adaption plans locally, the federal government will commit $\$ 1$ towards this fund for implementing these plans. The summary of attributes and their corresponding levels are presented in Table 2.1. Appendix A provides an example of a short-term choice card shown to respondents, and Appendix B provides an example of a long-term choice card with matching fund shown to respondents.

The third and last part of the survey contained information on respondents' socio-economic characteristics, including age, gender, level of education, level of income, employment status, household size and number of children in the household. These characteristics are also of our interests in order to capture and explain heterogeneity in responses by respondents.

\subsection{Sample Characteristics}

\subsubsection{Survey Data}

The survey was carried out in July 2014 over a period of four weeks, and a total of 814 individuals completed the survey with qualification. According to the survey results, approximately $73 \%$ of the respondents were identified as yearlong residents and the remaining $27 \%$ as seasonal residents. About $96 \%$ of the yearlong residents remained in Florida for all 12 months of the year while the majority of the seasonal residents stayed for 6 months. Due to incomplete information, 748 respondents are used in this study, with 550 yearlong residents and 198 seasonal residents. 
Although previous literature suggested that yearlong residents are normally more conscientious about their impacts on the environment relative to seasonal residents, our results from the first part of the survey find that seasonal residents had a relatively higher risk perception compared to yearlong residents. As presented in Figure 2.3, approximately $69 \%$ of the seasonal respondents agreed (or slightly agreed) on the statement that increased sea level rise is real and we will experience impacts in the short-term future, while only $55 \%$ of yearlong respondents agreed. The level of agreement for seasonal respondents was also consistent (from 69\% to 70\%) regarding the statement that sea level rise is real and we will experience impacts in the longterm future. Although a slightly higher portion of yearlong respondents perceived the SLR impacts in the long term (from 55\% to 59\%), their perceptions were still lower than seasonal respondents. Also, more yearlong respondents disagreed with the above statements compared to seasonal respondents.

In accordance with the higher risk perceptions, seasonal respondents were also found to have a higher level of concern about the projected impacts of sea level rise. Figure 2.4 presents the differences in the levels of concern between yearlong and seasonal respondents. The majority of both groups rated a moderate level of concern about SLR risks. There were a higher portion of seasonal respondents (25\%) who rated high concern about the issue of SLR compared to yearlong respondents $(14 \%)$. In contrast, a higher portion of yearlong respondents $(17 \%)$ rated no concern compared to seasonal respondents $(7 \%)$.

The respondents' average age was 52 years old and $48 \%$ of the respondents were male. Most respondents (40\%) fall in the age group of 45 - 64 years. About $50 \%$ of the respondents were employed or self-employed and $13 \%$ unemployed, also, $32 \%$ of them were retired. The average family size was 2.5 , which was slightly higher than the Florida average (2.48). About $26 \%$ of the respondents had children in the 
household. It is worth noting that seasonal respondents had a higher educational attainment and a higher income level compared to yearlong respondents. Yearlong respondents who had high school diploma or lower totaled $50 \%$, and another $26 \%$ of them had a Bachelor's degree at the time of survey. In the case of seasonal respondents, only $15 \%$ had high school diploma or lower and $47 \%$ had a college degree. The average annual income level was $\$ 40,000$ to $\$ 49,000$ for yearlong respondents and $\$ 60,000$ to $\$ 75,000$ for seasonal respondents. Overall, our sample is believed to be representative for the Florida community based on respondents' demographic composition. Detailed descriptive statistics of respondent characteristics are summarized in Table 2.2.

\subsubsection{GIS Data}

Spatial information for surveyed respondents used in this study was obtained using GIS analysis. To begin with, 1,747 grids $\left(10^{*} 10 \mathrm{~km}\right.$ each) were generated to cover all of Florida. Figure 2.5 illustrates the spatial distribution of surveyed respondents by geocoding their locations based on latitude and longitude information provided by GfK. ${ }^{1}$ The majority of the respondents in the survey are spread along the coastline as our study's geographic focus, with several clusters found in Florida's major cities labeled in the map, including Pensacola, Tampa, Jacksonville, Gainesville, Orlando, West Palm Beach, Fort Lauderdale, and Miami. The figure also shows the locations of yearlong and seasonal respondents, indicated by green and red circles, respectively. Given such spatial distribution, we believe that our sample and both the subsamples are representative of the population of coastal communities in Florida.

\footnotetext{
${ }^{1} \mathrm{~A}$ few respondents were geocoded using zip code information if their latitude and longitude data were not available.
} 
Figure 2.5 also provides an elevation map of Florida and the source of data was obtained from Florida Geographic Data Library (FGDL). The original dataset represents a five meter cell size Digital Elevation Model (DEM, 2013) covering the State of Florida, and it was then spatially joined into the $10^{*} 10 \mathrm{~km}$ grids presented in the figure. As shown, South Florida lies at a lower elevation than northern Florida, and much of the state is at or near sea level. Given the specific location of each surveyed respondent, the elevation information can be extracted consequently. Furthermore, the distance information can also be obtained by measuring each respondent's location to its nearest coastline. Note that there seems to be a strong correlation between elevation and distance to the coastline in Florida.

In order to examine the impacts of sea level rise on surveyed respondents in the short and long term, an SLR Inundation Surface Calculator coupled in GIS spatial analyst was used to calculate potential inundation every 10 years in Florida (also see Figure 2.1). ${ }^{2}$ The calculator uses the USACE sea level change projection methodology along with National Oceanic and Atmospheric Administration (NOAA) tide gauge data and sea level trends. Figure 2.6 presents the potential inundation and the consequently affected respondents in year 2030 (representing the short term) and and year 2060 (representing the long term). The calculation was based on the high projection curve with tidal datum values as Mean Higher High Water (MHHW) at the Key West tide gauge station. The calculated inundation output was then spatially joined into the $10^{*} 10 \mathrm{~km}$ grids and the area of inundation at each grid can thus be obtained. If the area of inundation had a positive value, the grid was considered as an inundation grid, and respondents who fell inside that grid were considered as affected respondents. In Figure 2.6, the affected respondents are rep-

\footnotetext{
${ }^{2}$ The tool is developed by University of Florida GeoPlan Center with funding from the Florida Department of Transportation Office of Policy Planning. Details can be found at: http://sls.geoplan.ufl.edu/\#intro.
} 
resented by orange circles and the unaffected respondents are represented by light green circles. Also, notice that more inundated grids, particularly in South Florida, can be observed in the long term (Map 2) than in the short term (Map 1). As a result, $55.7 \%$ were identified as affected respondents at year 2030 and $58.4 \%$ at year 2060. Detailed descriptive statistics from both the survey data and GIS data are summarized in Table 2.3.

\subsection{The Choice Experiment}

The choice experiment method is based on the Random Utility Theory (RUT), in which individuals are assumed to select the alternative that yields the highest utility. Specifically, an individual, labeled as $n$, faces a choice among $J$ alternatives. Then he or she would obtain a certain level of utility from each alternative. The utility that individual $n$ obtains from alternative $j$ is $U_{n j}$, where $j=1, \ldots, J$. Because the individual chooses an alternative that provides the greatest utility, the behavioral model is: choose alternative $i$ if and only if $U_{n i}>U_{n j} \forall j \neq i$ (Train, 2009).

As proposed by McFadden (1974) and Train (2009), we cannot observe individual's utility, but we can observe some attributes of the alternatives faced by the individual and some attributes of the individual. The indirect utility function is therefore derived and represented as:

$$
U_{n i}=V_{n i}+\varepsilon_{n i}
$$

where $V_{n i}$ is the observable or measurable component of utility, and $\varepsilon_{n i}$ is the random component that captures factors that affect utility but are not included in $V_{n i}$. The logit model can be obtained by assuming that each $\varepsilon_{n i}$ is an independently, identically distributed extreme value. 
The probability that individual $n$ chooses alternative $i$ depends on the fact that the utility provided by alternative $i$ is the highest among any other options $j$ :

$$
\begin{aligned}
P_{n i} & =\operatorname{Prob}\left(U_{n i}>U_{n j} \forall j \neq i\right) \\
& =\operatorname{Prob}\left(V_{n i}+\varepsilon_{n i}>V_{n j}+\varepsilon_{n j} \forall j \neq i\right) \\
& =\operatorname{Prob}\left(\varepsilon_{n j}-\varepsilon_{n i}<V_{n i}-V_{n j} \forall j \neq i\right)
\end{aligned}
$$

The observable component of utility $V_{n i}$ is usually specified to be linear in parameters, such that:

$$
U_{n i}=\sum_{k} \beta_{n i k} X_{n i k}+\varepsilon_{n i}
$$

where $X_{n i k}$ is a vector of $K$ choice-related characteristics consisted of observed choice attributes and individual characteristics, and $\beta_{\text {nik }}$ is a vector of $K$ parameters to be estimated.

In this study, individuals make a choice between two alternative SLR adaptation plans (basic and extensive) compared to the status quo option. Adapting and preparing for SLR can be realized at certain costs to be paid in annual tax and the cost of not adapting to SLR is zero. Based on this setting, equation (2.3) can be rewritten as:

$$
U_{n i}=\alpha+\beta_{\alpha} A_{n i}+\beta_{p} P_{n i}+\beta_{h} H_{n i}+\varepsilon_{n i}
$$

where $\alpha$ is the alternative specific constant (ASC), $\beta_{\alpha}$ is the vector of coefficients to choice attributes $A, \beta_{p}$ is the coefficient of the payment attribute $P$, and $\beta_{h}$ is the vector of coefficients related to the household characteristics $H$, including attitudinal factors and socio-economic factors.

We test three specific hypotheses in this study. Based on the work by Schaafsma et al. (2013), the first hypothesis is related to spatially heterogeneous preferences. Spatial heterogeneity is measured in this study through the distance-decay effect, elevation effect, and inundation effect. The distance-decay effect is the effect of 
Euclidean distance from individual $n$ to his or her nearest coast $c$. The elevation effect is omitted in this hypothesis section because it is highly correlated with distance effect and will be discussed later in the result section. Here, the inundation effect uses a dummy variable which takes the value 1 if an individual $n$ is located near a coast $c$ that will be inundated as a result of the projected SLR, denoted Inund $_{n c}$. Similarly, an individual $n$ who resides at a non-inundated area from the projected SLR is denoted non-Inund ${ }_{n c}{ }^{3}$ Differences in the distance-decay and between Inund and non-Inund can be examined by adding the spatial heterogeneity function $f\left(\right.$ Dist $_{n c}$, Inund $\left._{n c}\right)=\beta_{d} \operatorname{Ln}\left(\right.$ Dist $\left._{n c}\right)+\beta_{i}$ Inund $_{n c}$ into equation $(2.4)^{4}$ :

$$
U_{n i}=\alpha+\beta_{\alpha} A_{n i}+\beta_{p} P_{n i}+\beta_{h} H_{n i}+\beta_{d} L n\left(\text { Dist }_{n c}\right)+\beta_{i} \text { Inund }_{n c}+\varepsilon_{n i}
$$

Therefore, the first null hypothesis is that there is no distance-decay effect on preferences among respondents and/or between inundated respondents and noninundated respondents.

$$
H_{1}^{0}: \beta_{d}=0 \text { and } \beta_{i}=0
$$

The null hypothesis can be rejected if spatially heterogeneous preferences exist, that is, respondents' distance to their nearest coast presents significant effects on their choice behaviors and/or the preferences between inundated and non-inundated respondents are very different from each other in the choice experiment.

The second hypothesis covers the unique aspects of Florida's resident composition and considers the differences and similarities in preferences between yearlong and seasonal residents. In doing so, an individual $n$ is first identified as either a

\footnotetext{
${ }^{3}$ The inundation effect presented here is comparable to the user/non-user dummy variable in Schaafsma et al. (2013), where $U_{s e r}{ }_{i j}$ in their work takes the value 1 if an individual $i$ has recreated at site $j$ prior to the survey.

${ }^{4}$ We have tested and compared the significance and impact on model fit for different functional forms based on Concu (2007). The log linear form of distance resulted in the best model fit and thus, is presented only in the specification.
} 
yearlong resident $y$ or a seasonal resident $s$, and equation (2.5) can be expanded as:

$$
\begin{aligned}
& U_{y i}=\alpha+\beta_{\alpha} A_{y i}+\beta_{p} P_{y i}+\beta_{h} H_{y i}+\beta_{d} \operatorname{Ln}\left(\text { Dist }_{y c}\right)+\beta_{i} \text { Inund }_{y c}+\varepsilon_{y i} \\
& U_{s i}=\alpha+\beta_{\alpha} A_{s i}+\beta_{p} P_{s i}+\beta_{h} H_{s i}+\beta_{d} \operatorname{Ln}\left(\text { Dist }_{s c}\right)+\beta_{i} \text { Inund }_{s c}+\varepsilon_{s i}
\end{aligned}
$$

The second null hypothesis is then presented as:

$$
H_{2}^{0}: \alpha_{y}=\alpha_{s}
$$

Although we can test and compare all the coefficients from equation (2.7), the alternative specific constants (ASC) offer a general idea of respondents' preferences and are thus tested. Rejection of the null hypothesis will imply that yearlong and seasonal residents perceive risks differently and have heterogeneous preferences towards sea level rise adaptation plans in Florida.

The third and last hypothesis relates to respondents' sensitivity to time horizons displayed in the choice experiment. Based on USACE's estimated projection, we utilize two time horizons: a short-term impact $(S T)$ and a long-term impact $(L T)$, and equation (2.5) can be expanded as:

$$
\begin{aligned}
& U_{n i}^{S T}=\alpha+\beta_{\alpha}^{S T} A_{n i}+\beta_{p}^{S T} P_{n i}+\beta_{h}^{S T} H_{n i}+\beta_{d}^{S T} \operatorname{Ln}\left(\text { Dist }_{n c}\right)+\beta_{i}^{S T} \text { Inund }_{n c}+\varepsilon_{n i} \\
& U_{n i}^{L T}=\alpha+\beta_{\alpha}^{L T} A_{n i}+\beta_{p}^{L T} P_{n i}+\beta_{h}^{L T} H_{n i}+\beta_{d}^{L T} L n\left(\text { Dist }_{n c}\right)+\beta_{i}^{L T} \text { Inund }_{n c}+\varepsilon_{n i}
\end{aligned}
$$

where $U_{n i}^{S T}$ is the utility obtained from choosing a short-term adaptation plan and $U_{n i}^{L T}$ from choosing a long-term adaptation plan. Accordingly, the third null hypothesis is that preferences in the two different terms are similar among respondents, in other words, people are not sensitive to time horizons.

$$
H_{3}^{0}: \alpha^{S T}=\alpha^{L T}
$$

The null hypothesis can be rejected if we find the short-term ASC and long-term ASC to be different, implying that people are sensitive to different time horizons when making a decision. 


\subsection{Choice Experiment Results}

\subsubsection{Results from the Short-term Choice}

Nine different short-term random utility choice models were estimated, and the results of which are presented in Table 2.4 and Table 2.5. Model 1 simply examines the effects of attributes on the choice outcome for all respondents. Model 2 is the extended model disregarding the spatial heterogeneity, and Models 3-6 are the same

as Model 2 but account for spatial heterogeneity, through examining the influence of distance, elevation, and inundation. Models 7-9 in Table 2.5 investigate the choice behaviors between yearlong and seasonal residents, including the basic model and the extended models with or without spatial heterogeneity.

In the basic "attribute only" model (see Model 1 in Table 2.4), the ASC is positive and significant at $10 \%$ level, indicating that respondents had some preferences towards choosing the short-term SLR adaptation plan (moving away from status quo). In addition, the coefficients of four choice attributes are significant with expected signs. Improvements in coastally vulnerable lands, low-lying and underground drainages, and pump stations all have positive effects on selecting the adaptation plan. Payment has a negative sign as expected, implying that the plan is less likely to be chosen if the cost is higher. The regulation attribute is found to be insignificant.

Model 2 is the extended model estimated through interacting respondents' perceptions and socio-economic variables with the ASC, in order to evaluate the influence of respondent characteristics on the choice behavior. The extended model presents improvements on the estimation in that the log-likelihood has decreased and $R^{2}$ has increased, indicating a better explanatory power of this model. Moreover, the extended models that accounted for spatial heterogeneity are also reported 
in Table 2.4. In Model 3, the distance variable is included and its coefficient has a significantly negative sign. Respondents who live closer to the coast have a higher probability to choose the adaptation plan and are willing to pay more for SLR risk mitigation. Model 4 shows the elevation effect on the choice behavior as an alternative spatial factor for distance. The significant and negative sign indicates that respondents who live at relatively higher elevations value the adaptation to SLR risks less. Lastly, Models 5 and 6 account for additional spatial heterogeneity and investigate the preferences between affected and unaffected respondents due to SLR inundation, both in the short-term and long-term future. The positive sign of $A S C^{*}$ Inund2030 implies that respondents who live in areas that will be inundated in year 2030 (short term) have a higher probability to choose the short-term adaptation plan compared to those unaffected respondents. In addition, the coefficient of $A S C^{*}$ Inund2060 is also significantly positive, indicating that respondents, who are expected to be affected in year 2060 (long term), also have a higher probability to choose the short-term adaptation plan. The significant distance-decay effects, elevation effects, and inundation effects in the estimation results demonstrate that there is a strong spatial heterogeneity in the sample, hence rejecting the first null hypothesis $\left(H_{1}^{0}: \beta_{d}=0\right.$ and $\left.\beta_{i}=0\right)$.

Regarding other respondent characteristics, similar results are observed across all the extended models (see Models 2-6 in Table 2.4). The coefficients of the interaction terms between ASC and matching fund, risk perception, concern and household income are statistically significant with expected signs. First, providing a matching fund by the federal government increases respondents' willingness to opt-in for the adaptation plan. This is evidence to support that implementing a matching fund program can incentivize people to select the plan or participate in the program. Second, the positive signs of $A S C^{*} \operatorname{Impact}(S R)$ and $A S C^{*}$ Concern 
indicate that respondents who were aware of the global warming induced SLR in the short term and who expressed a higher level of concern are more willing to accept the adaptation plan. On the contrary, respondents who believed that increased SLR is not real and there will be no impact are less likely to accept the plan, indicated by the negative sign of $A S C^{*} \operatorname{Impact}(N o)$. In line with the existing literature, perceptions and attitudes can affect people's recognition in environmental problems, and people tend to take some actions to avoid threats of adverse environmental events that they believe to be serious and probable (Patchen, 2006). Finally, higher household income increases the probability of choosing the SLR adaptation plan. Richer households are generally willing to invest more to adapt themselves for adverse environmental impacts.

In this study, we are also interested in investigating whether seasonal residents perceive the SLR risks differently from yearlong residents. In doing so, a dummy variable Yearlong, which takes the value 1 for yearlong respondents, was utilized and added to the extended models in Table 2.4. The significantly negative sign of $A S C^{*} Y$ earlong (except for Model 4) indicates that compared to seasonal residents, yearlong residents are less likely to support the adaptation plan. On the one hand, the influence of place attachment did not play an important role in yearlong residents' decisions to promote an adaptation plan. On the other hand, higher risk perceptions and higher income levels may have contributed positively to seasonal residents' decisions to select the adaptation plan. Based on this finding, we can therefore reject the second null hypothesis that yearlong and seasonal residents have the same preference $\left(H_{2}^{0}: \alpha_{y}=\alpha_{s}\right)$ in the case of short-term choice.

Furthermore, respondents were estimated separately based on their residency status, in order to further examine the differences and similarities in preferences between the two groups. The estimation results are presented in Table 2.5. According 
to the basic "attribute only" model (Model 7), the ASC remains positively significant for seasonal respondents but insignificant for yearlong respondents, which is consistent with the results when using the dummy variable Yearlong. Seasonal residents value the SLR adaptation options in a positive way, possibly stemming from their higher risk perceptions and higher household income. The last two columns in Table 2.5 present the extended models for both groups with or without spatial heterogeneity. Contradicting with the result in Model 7, the ASC becomes significant and negative for yearlong respondents in Model 8. This is evidence to show that omission of spatial preference heterogeneity may produce biased and inconsistent parameters on the estimation result. Moreover, Model 9 that accounted for spatial heterogeneity tends to reduce the biased consequence observed in Model 8, as the ASCs all become insignificant. In Model 9, strong distance-decay effects are observed for both groups, indicated by the significant interaction terms between ASC and the distance variable.

Differences in the respondent characteristics in determining preferences between the two groups can also be observed in the extended models. Besides the matching fund and risk perception variables, household concern and household income appear to be insignificant determinants for the two separated groups, indicating that the influence of risk perceptions is more robust compared to the influence of income levels in our sample. In addition, age is found to be a significant determinant for seasonal respondents while choosing an adaptation plan. The negative sign implies that younger people among seasonal residents are more willing to adapt to SLR. Lastly, employment status is found to be an important factor for yearlong respondents. The positive interaction term indicates that employed (or self-employed) yearlong residents are more likely to promote an adaptation plan compared to unemployed or retired yearlong residents. 


\subsubsection{Results from the Long-term Choice}

Utilizing the same setting from the short-term case, another nine different random utility choice models were estimated for the long term, and the results of which are presented in Table 2.6 and Table 2.7. Based on the "attribute only" model (see Model 1 in Table 2.6), the coefficients of all choice attributes are significant with expected signs. Improvements in coastal wetland, reductions of flood risks, and more strict property regulations all have positive effects on the choice outcome. Payment has a negative sign as expected, implying a negative effect on respondents' utility. Also, note that the ASC becomes negative in this model, indicating that respondents had no preference for choosing the long-term SLR adaptation plan (staying at status quo). Given the fact that respondents have different preferences between the short-term and long-term adaptation plan, our third null hypothesis is rejected $\left(H_{3}^{0}: \alpha^{S T}=\alpha^{L T}\right)$.

Models 2-6 in Table 2.6 are the extended models with or without the inclusion of spatial heterogeneity. Similar to the results in the short-term choice models, the negative signs of the interaction terms between ASC and Yearlong suggest that yearlong residents are more likely to vote against the long-term adaptation plan. The distance and elevation variables are found to be significant and negative, presented in Models 3 and 4. Respondents who live closer to the coast or at relatively lower elevations have a higher probability to choose the long-term adaptation plan. However, no significant differences in preferences are found between unaffected and affected respondents due to SLR inundation in the short-term future (see Model 5). As for the SLR inundation in the long term, the affected respondents are more willing to select the adaptation plan, but only at the $10 \%$ significance level (see Model 6). As a result, in this case of long-term choice, we can reject the first null hypothesis that there is no spatial heterogeneity measured by distance effects $\left(H_{1}^{0}\right.$ : 
$\left.\beta_{d}=0\right)$, but fail to reject the null hypothesis that there is no spatial heterogeneity measured by inundation effects at a large significance level $\left(H_{1}^{0}: \beta_{i}=0\right)$.

Regarding other respondent characteristics, similar results are observed in the estimation results compared to the results in the short-term choice. Besides that $A S C^{*} F$ und $(L R)$ becomes an insignificant determinant in the long-term choice outcome, the coefficients of the interaction terms between ASC and risk perception, concern, and household income remain statistically significant with expected signs. The positive signs of $A S C^{*} \operatorname{Impact}(L R)$ and $A S C^{*}$ Concern indicate that respondents who were aware of the global warming induced SLR in the long term and who expressed a higher level of concern are more willing to accept the adaptation plan. On the contrary, respondents who believed that increased SLR is not real and there will be no impact are less likely to accept the adaptation plan, indicated by the negative sign of $A S C^{*} \operatorname{Impact}\left(N_{o}\right)$. Richer households are still more willing to pay for the adaptation plan in the long term.

Next, we examine the differences and similarities in preferences between seasonal and yearlong residents, and the estimation results are presented in Table 2.7. According to the "attribute only" model (Model 7), the ASCs are negative for both seasonal and yearlong respondents. This suggests that the two groups have the same preference that they do not support the long-term SLR adaptation plan. Based on this finding, we fail to reject the second null hypothesis for the two groups $\left(H_{2}^{0}\right.$ : $\alpha_{y}=\alpha_{s}$ ) in the long term. Model 8 in Table 2.7 is the extended model disregarding the spatial heterogeneity. The omission of spatial factors tends to overestimate the negative effects on respondents' preferences, indicated by the large magnitude of the ASC. Model 9 therefore provides better estimation results after accounting for spatial heterogeneity. Turning to the interaction terms between ASC and spatial factors presented in Model 9, it is interesting to find that there is still a strong spatial 
heterogeneity observed for yearlong respondents when measured by distance-decay effects. However, no spatial heterogeneity can be observed anymore for seasonal respondents. This finding could be explained by the fact that seasonal residents are less knowledgeable or less familiar with the local climate change situations; therefore, they do not have an idea on how their spatial locations will be impacted in the long-term future. Also, as suggested in the literature, seasonal residents are relatively more willing to relocate their property in the future compared to yearlong residents, and if this is true, spatial factors may not be influential to seasonal residents when making a long-term choice.

Finally, the results show that risk perception, concern, and household income are still significant determinants for yearlong respondents while choosing an adaptation plan. On the other hand, household concern is the only respondent characteristic that appeares to be a significant determinant of choice behavior for seasonal respondents. None of the other respondent attitude characteristics (e.g., risk perception) or demographic characteristics (e.g., age or income) have a significant impact on seasonal residents' preferences.

\subsection{WTP and Economic Values for SLR Adaptation Plan}

The marginal willingness to pay (MWTP) estimates for each choice attribute that mitigates sea level rise risks in Florida are presented in Table 2.8. The calculations

are based on the basic "attribute only" model and the extended model with the distance variable. The results show that the average household in the combined sample is willing to pay $\$ 67$ - 71 for vulnerable land purchase, $\$ 95$ - 99 for drainage relocation, and $\$ 60$ - 69 for new pump stations included in the short-term adaptation plan. Turning to the long-term adaptation plan, the results indicate that the average 
household in the combined sample is willing to pay $\$ 154$ - 164 for wetland restoration and $\$ 138$ - 161 for flood risk reduction. In general, respondents find relocation of low-lying and underground drainages to be the most valued line of adaptation in the short term, and wetland restoration is the most valued adaptation strategy in the long term.

Results from the two separated groups indicate that yearlong residents in Florida are willing to pay $\$ 54$ - 61 per household for vulnerable land purchase, $\$ 100$ - 114 for underground drainage relocation, and $\$ 30$ - 33 for new pump stations in the short term. Also, yearlong residents are willing to pay about $\$ 133$ per household for wetland restoration and $\$ 93$ - 107 for flood risk reduction in the long term. The results for Florida's seasonal residents are also presented. Accordingly, seasonal residents are willing to pay more than yearlong residents (\$116 - 128 per household for vulnerable land purchase, $\$ 73$ - 167 for underground drainage relocation, and $\$ 167$ - 200 for new pump stations in the short term; $\$ 221$ - 325 per household for wetland restoration and $\$ 314$ - 399 for flood risk reduction in the long term). The differences are most pronounced in the installation of new pump stations and could reflect a higher income level for seasonal residents relative to yearlong residents.

Under the assumption that our sample is representative of Florida's population, a tentative estimate of economic value for sea level rise adaptation projects in Florida can be calculated. By aggregating over all Florida's taxpaying households ${ }^{5}$ over the next 10 years, the economic values for short-term SLR adaptation plan are approximately $\$ 5.3$ billion (95\% CI: $\$ 0.4$ - 10 billion) for coastally vulnerable land purchase, $\$ 7.3$ billion (95\% CI: $\$ 2$ - 12 billion) for underground drainage relocation, and $\$ 5.3$ billion (95\% CI: $\$ 0.5$ - 10 billion) for installation of new pump stations.

\footnotetext{
${ }^{5}$ According to the Bureau of Economic and Business Research (BEBR, 2014) at the University of Florida, there were 7,669,541 households in Florida by April 1, 2014. Details see: https://www.bebr.ufl.edu/sites/default/files/Research $\ \% 20$ Reports/households_2014.pdf
} 
Also, by aggregating over all Florida's taxpaying households over the next 20 years, the economic values for long-term SLR adaptation plan are approximately $\$ 25.1$ billion (95\% CI: $\$ 11$ - 40 billion) for wetland restoration, and $\$ 24.6$ billion (95\% CI: $\$ 10$ - 39 billion) for flood risk reduction. These estimates are expected to provide valuable information for decision makers as they analyze sea level rise adaptation and mitigation projects in Florida. A comprehensive cost-benefit analysis can also be conducted for future research.

\subsection{Conclusion}

Coastal communities cannot escape the need to think hard about what sea level rise means to them. The issue really falls at the junction of planning and politics, because it involves a series of judgements about what might happen to sea levels, how it will affect low-lying areas, and how the possible decisions about defenses against the worst effects might stack up against other spending priorities and the interest groups which support them (Ince, 1990). In this study, we employ choice modelling technique to understand household perceptions and preferences and provide estimates of economic values for SLR adaptation projects in Florida. Our choice experiment took place in July 2014, and aimed to examine Florida's yearlong and seasonal residents' preferences regarding SLR adaptation strategy for the short term and long term.

Our empirical results first identify the need to account for spatial heterogeneity in environmental valuation, thus rejecting the first null hypothesis. Respondents who live closer to the coastline, live at relatively lower elevations, and live in the expected "inundation zones" are more likely to accept the plans. The results also support the notion that there are attitudinal and demographic differences in respon- 
dents' preferences for environmental improvements. Respondents with higher risk perceptions, more concerns, and higher income levels are generally more willing to promote the adaptation plans. Moreover, the results indicate that there are heterogeneous preferences between yearlong and seasonal respondents in Florida, and our second null hypothesis can be rejected. Seasonal respondents are found to be willing to pay more for the adaptation plans compared to yearlong respondents, stemming from their higher risk perceptions and higher income. Lastly, our results indicate that respondents are very sensitive to the time horizons utilized in the experiment, thus rejecting the third null hypothesis. In general, people from selected communities in Florida support the short-term SLR adaptation strategy within the next 10 - 20 years, but not the long-term adaptation strategy within the next 30 - 50 years.

The results also provide some policy implications. First, information about the long-term impacts of climate change-induced sea level rise and the choice of effective adaptation strategies should be emphasized in order to raise public awareness. Accordingly, the local governments, institutions, and social media should work together and take initiatives to address the adverse impacts of climate change to future generations, or come up with more incentivized programs for the long-term adaptation projects. Second, it is necessary to integrate place of residence or residency status considerations into the adaption policies. Based on our results, more effective policies should be designed in a way that account for spatial preference heterogeneity. For example, two different payment strategies could be employed between coastal residents and inland residents. Third, the result that lower income respondents are less likely to pay for the plan suggests that income inequality is a major impediment for a program to be successfully carried out. Therefore, it is important to focus on socially vulnerable groups within communities and provide them with necessary discounts or subsidies when implementing the adaptation plan. 
Finally, the expected contributions of this study to the literature are twofold. First, it complements the limited studies on the public preferences for climate change adaptation. To our knowledge, this study is the first attempt to understand the SLR issue in Florida from a household perspective. It also covers the unique feature regarding Florida's composition of residents and gives special attention on seasonal residents' preferences towards SLR adaptation options. Second, it adds to the increasing number of choice experiment studies in the climate adaptation literature. In particular, it contributes to the literature which estimates the economic values of improved infrastructures and enhanced climate stability. 


\section{References}

Barbier, E. B., Hacker, S. D., Kennedy, C., Koch, E. W., Stier, A. C., \& Silliman, B. R. (2011). The value of estuarine and coastal ecosystem services. Ecological Monographs, 81(2), 169-193. doi:10.1890/10-1510.1

Bates, B., Kundzewicz, Z., Wu, S., \& Palutikof, J. (2008). Climate change and water. Technical paper of the Intergovernmental Panel on Climate Change, IPCC Secretariat, Geneva.

Bureau of Economic and Business Research (BEBR). (2014). Households and average household size in Florida: April 1, 2014. Florida Population Studies, University of Florida, Miami, FL. Available Online: Https://Www.Bebr.Ufl.Edu/ Sites/Default/Files/Research $\backslash \% 20 R e p o r t s / h o u s e h o l d s \_2014 . P d f$

Beckmann, M. J. (1999). Lectures on location theory. Berlin; New York: Springer Verlag.

Bergmann, A., Colombo, S., \& Hanley, N. (2008). Rural versus urban preferences for renewable energy developments. Ecological Economics, 65(3), 616-625. doi: http://dx.doi.org/10.1016/j.ecolecon.2007.08.011

Berk, R. A., \& Fovell, R. G. (1999). Public perceptions of climate change: A willingness to pay' assessment. Climatic Change, 41(3), 413-446. doi:10.1023/A:1005489221644

Birol, E., Koundouri, P., \& Kountouris, Y. (2009). Using the choice experiment method to inform flood risk reduction policies in the upper Silesia region of Poland. Land-use and Natural Resources: Context of Disaster Reduction and Sustainability.

Brouwer, R., Martin-Ortega, J., \& Berbel, J. (2010). Spatial preference heterogeneity: A choice experiment. Land Economics, 86(3), 552-568.

Brouwer, R., \& Schaafsma, M. (2012). Modelling risk adaptation and mitigation behaviour under different climate change scenarios. Climatic Change, 117(1), 11-29. doi: 10.1007/s10584-012-0534-1

Burley, D. M. (2010). Losing ground: Identity and land loss in coastal Louisiana. Jackson: University Press of Mississippi. 
Concu, G. B. (2007). Investigating distance effects on environmental values: A choice modelling approach. Australian Journal of Agricultural and Resource Economics, 51(2), 175-194. doi: 10.1111/j.1467-8489.2007.00381.x

Costanza, R., Pérez-Maqueo, O., Martinez, M. L., Sutton, P., Anderson, S. J., \& Mulder, K. (2008). The value of coastal wetlands for hurricane protection. Ambio, $37(4), 241-248$.

Digital Elevation Model (DEM). (2013). Florida digital elevation model (DEM) mosaic - 5-meter cell size - elevation units feet. University of Florida GeoPlan Center, Gainesville, FL.

Florida Oceans and Coastal Council. (2010). Climate change and sea-level rise in Florida: An update of "The effects of climate change on Florida's ocean and coastal resources". 2009 Report, Tallahassee, Florida. Available online:www.Floridaoceanscouncil.Org.

Gedan, K. B., Kirwan, M. L., Wolanski, E., Barbier, E. B., \& Silliman, B. R. (2010). The present and future role of coastal wetland vegetation in protecting shorelines: Answering recent challenges to the paradigm.Climatic Change, 106(1), 7-29. doi:10.1007/s10584-010-0003-7

Green, G. P., Marcouiller, D., Deller, S., Erkkila, D., \& Sumathi, N. R. (1996). Local dependency, land use attitudes, and economic development: Comparisons between seasonal and permanent Residents. Rural Sociology, 61(3), 427-445. doi:10.1111/j.1549-0831.1996.tb00627.x

Hanson, S., Nicholls, R., Ranger, N., Hallegatte, S., Corfee-Morlot, J., Herweijer, C., \& Chateau, J. (2010). A global ranking of port cities with high exposure to climate extremes. Climatic Change, 104(1), 89-111. doi:10.1007/s10584-010-9977-4

Harlem, P. (2008). Sea level rise map projections. Sea Level Rise Collection Paper 2, Florida International University. Available online: http://Digitalcommons.Fiu.Edu/Sea_level_rise/2

Ince, M. (1990). The rising seas. In Earthscan L. (Ed.), London, UK.

Intergovernmental Panel on Climate Change. (2007). Climate change 2007: Impacts, adaptation and vulnerability. Contribution of Working Group II to the Fourth Assessment Report of the Intergovernmental Panel on Climate Change, M.L. Parry, O.F. Canziani, J.P. Palutikof, P.J. Van Der Linden and C.E. Hanson, Eds., Cambridge University Press, Cambridge, UK. 
Johnston, R. J., Swallow, S. K., \& Bauer, D. M. (2002). Spatial factors and stated preference values for public goods: Considerations for rural land use. Land Economics, 78(4), 481-500. doi: 10.2307/3146848

Jørgensen, S. L., Olsen, S. B., Ladenburg, J., Martinsen, L., Svenningsen, S. R., \& Hasler, B. (2013). Spatially induced disparities in users' and non-users' WTP for water quality improvements - Testing the effect of multiple substitutes and distance decay. Ecological Economics, 92, 58-66. doi: http://dx.doi.org/10.1016/j.ecolecon.2012.07.015

Kloos, J., \& Baumert, N. (2014). Preventive resettlement in anticipation of sea level rise: A choice experiment from Alexandria, Egypt. Natural Hazards, 76(1), 99-121. doi: 10.1007/s11069-014-1475-y

Landry, C. E., Hindsley, P., Bin, O., Kruse, J. B., Whitehead, J. C., \& Wilson, K. (2011). Weathering the storm: Measuring household willingness-to-pay for risk-reduction in post-Katrina New Orleans. Southern Economic Journal, 77(4), 991-1013. doi: 10.4284/0038-4038-77.4.991

Lausche, B., \& Maier, L. (2013). Sea level rise adaptation: Emerging lessons for local policy development. Technical Report No. 1723, Sarasota, FL: Mote Marine Laboratory. Available Online: https://Dspace.Mote.Org/Dspace/Handle/2075/3223

Layton, D. F., \& Brown, G. (2000). Heterogeneous preferences regarding global climate change. Review of Economics and Statistics, 82(4), 616-624. doi: $10.1162 / 003465300559091$

Lee, J. J., \& Cameron, T. A. (2008). Popular support for climate change mitigation: Evidence from a general population mail survey. Environmental and Resource Economics, 41(2), 223-248. doi:10.1007/s10640-007-9189-1

McFadden, D. (1974). Conditional logit analysis of qualitative choice behavior. In Frontiers in Econometrics, ed. P. Zarembka, New York: Academic Press.

Mozumder, P., Maina, S., Rodriguez, A. M., Seeteram, N., \& Tahsin, S. (2014). Sea-level rise, critical infrastructure, and coastal vulnerability: Investigating year-long and seasonal residents' perceptions and preferences for risk mitigation. Report Prepared for Florida Department of Community Affairs, Division of Emergency Management. International Hurricane Research Center, Miami, Florida. 
Office of Legislative and Governmental Affairs. (2014). Testimony of the honorable philip levine mayor of the city of Miami beach, Florida before the U.S. senate committee on commerce, science and transportation subcommittee on science and space. National Aeronautics and Space Agency (NASA).

Patchen, M. (2006). Public attitudes and behavior about climate change - What shapes them and how to influence them. Purdue Climate Change Research Centre. Available Online:https://Www.Purdue.Edu/Discoverypark/Climate/Resources/ Docs/Patchen $\backslash \% 200 P 0601 . P d f$

Remoundou, K., Diaz-Simal, P., Koundouri, P., \& Rulleau, B. (2015). Valuing climate change mitigation: A choice experiment on a coastal and marine ecosystem. Ecosystem Services, 11, 87-94. doi: http://dx.doi.org/10.1016/j.ecoser.2014.11.003

Schaafsma, M., Brouwer, R., Gilbert, A., van den Bergh, J., \& Wagtendonk, A. (2013). Estimation of distance-decay functions to account for substitution and spatial heterogeneity in stated preference research. Land Economics, 89(3), 514-537.

Schaafsma, M., Brouwer, R., \& Rose, J. (2012). Directional heterogeneity in WTP models for environmental valuation. Ecological Economics, 79, 21-31. doi: http://dx.doi.org/10.1016/j.ecolecon.2012.04.013

Scotchmer, S., \& Thisse, J. (1999). Space and value: An outlook and new perspectives. Oxford: Oxford University Press. doi:10.1093/0198292112.003.0010

Shugar, K., \& Obeysekera, J. (2010). Climate change and sea level rise planning and adaptation strategies. Governing Board Joint Workshop with the Water Resources Advisory Commission (WRAC), South Florida Water Management District, West Palm Beach, FL.

Smith, C. (2012). Miami Beach: Public works- storm water. City of Miami Beach Storm water Management Master Plan- Executive Summary. Available Online: http://Miamibeachfl.Gov/Publicworks/Scroll.Aspx?id=27280

Snider, A., Hill, J., Luo, S., Buerger, B., \& Herstine, J. (2011). Implications for place attachment in coastal reserve management. Ocean $\&$ Coastal Management, 54 (8), 612-620. doi:http://dx.doi.org/10.1016/j.ocecoaman.2011.06.002

Stouffer, S. A. (1940). Intervening opportunities: A theory relating mobility and distance. American Sociological Review, 5(6), 845-867. 
Tait, P., Baskaran, R., Cullen, R., \& Bicknell, K. (2012). Nonmarket valuation of water quality: Addressing spatially heterogeneous preferences using GIS and a random parameter logit model. Ecological Economics, 75, 15-21.

doi:http://dx.doi.org.ezproxy.fiu.edu/10.1016/j.ecolecon.2011.12.009

Train, K. (2009). Discrete choice methods with simulation (2nd ed). Cambridge; New York: Cambridge University Press.

Southeast Florida Regional Climate Change Compact Technical ad hoc Work Group (2011). A unified sea level rise projection for southeast Florida. A Document Prepared for the Southeast Florida Regional Climate Change Compact Steering Committee. Available Online:

http://Www.Southeastfloridaclimatecompact.Org//Wp-Content/Uploads/2014/ 09/Sea-Level-Rise.Pdf

Viscusi, W. K., \& Zeckhauser, R. J. (2006). The perception and valuation of the risks of climate change: A rational and behavioral blend. Climatic Change, 77(1), 151-177. doi:10.1007/s10584-006-9075-9

Wang, B., Chen, S., Zhang, K., \& Shen, J. (1995). Potential impacts of sea-level rise on the Shanghai area. Journal of Coastal Research, Special Issue No14, 151-166.

Whitehead, J. C., Poulter, B., Dumas, C. F., \& Bin, O. (2009). Measuring the economic effects of sea level rise on shore fishing. Mitigation and Adaptation Strategies for Global Change, 14(8), 777-792. doi: 10.1007/s11027-009-9198-1 


\section{Tables}

Table 2.1: Attribute Levels Used in the Survey

\begin{tabular}{c|l|ll}
\hline \hline $\begin{array}{l}\text { Time } \\
\text { Horizon }\end{array}$ & Attribute & Description & Levels \\
\hline & & & \\
Short & Drainage & Relocation of low-lying or underground drainage & $0,30 \%, 40 \%, 50 \%, 60 \%$ \\
Term & Pump & Installation of new pump stations & $0,10,20,40,50$ \\
$(10-20$ & Regulation & New regulation for raising elevation requirements & 4 feet, 3 feet, 2 feet \\
years $)$ & Payment & Payment (annual local tax over the next 10 years) & $\$ 0, \$ 30, \$ 40, \$ 60, \$ 80$ \\
\hline Long & Wetland & Coastal wetland restoration & $0,30 \%, 40 \%, 50 \%, 60 \%$ \\
Term & Flood & Urban flood reduction & $0,30 \%, 40 \%, 50 \%, 60 \%$ \\
$(30-50$ & Regulation & New regulation for raising elevation requirements & 4 feet, 3 feet, 2 feet \\
years) & Payment & Payment (annual local tax over the next 20 years) & $\$ 0, \$ 40, \$ 50, \$ 80, \$ 100$ \\
\hline \hline
\end{tabular}


Table 2.2: Descriptive Statistics of Respondent Characteristics

\begin{tabular}{|c|c|c|c|}
\hline & Combined & Yearlong & Seasonal \\
\hline Average age (years) & 52.4 & 54.7 & 46.1 \\
\hline \multicolumn{4}{|l|}{ Gender $(\%)$} \\
\hline Male & 47.7 & 50.7 & 39.4 \\
\hline Female & 52.3 & 49.3 & 60.6 \\
\hline \multicolumn{4}{|l|}{ Level of education (\%) } \\
\hline Less than high school & 6.1 & 7.5 & 2.5 \\
\hline High school & 34.2 & 42.2 & 12.1 \\
\hline Some college & 27.8 & 24.0 & 38.4 \\
\hline Bachelor's degree or higher & 31.8 & 26.4 & 47.0 \\
\hline \multicolumn{4}{|l|}{ Level of income (\%) } \\
\hline Less than $\$ 19,999$ & 12.7 & 14.7 & 7.1 \\
\hline$\$ 20,000$ to $\$ 100,000$ & 66.0 & 66.5 & 64.6 \\
\hline$\$ 100,000$ or more & 21.3 & 18.7 & 28.3 \\
\hline \multicolumn{4}{|l|}{ Employment status (\%) } \\
\hline Employed/Self employed & 50.3 & 46.7 & 60.1 \\
\hline Unemployed & 12.6 & 14.4 & 7.6 \\
\hline Retired & 32.2 & 33.1 & 29.8 \\
\hline Other & 4.9 & 5.8 & 2.5 \\
\hline Average household size (people) & 2.5 & 2.5 & 2.6 \\
\hline Households with children (\%) & 26.1 & 23.6 & 32.8 \\
\hline Obs & 748 & 550 & 198 \\
\hline
\end{tabular}


Table 2.3: Descriptive Statistics of Other Respondent Information

\begin{tabular}{|c|c|c|c|c|c|}
\hline & \multirow[t]{2}{*}{ Variable } & \multirow[t]{2}{*}{ Description } & \multicolumn{3}{|c|}{ Mean (Standard Deviation) } \\
\hline & & & Combined & Yearlong & Seasonal \\
\hline \multirow{4}{*}{ 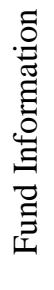 } & \multirow{3}{*}{ Fund(SR) } & \multirow{4}{*}{$\begin{array}{l}\text { The federal government will commit a } \\
\text { matching fund for implementing the short } \\
\text { term adaptation plans. (=1 if yes, } 0 \text { if no) } \\
\text { The federal government will commit a } \\
\text { matching fund for implementing the long } \\
\text { term adaptation plans. (= } 1 \text { if yes, } 0 \text { if no) }\end{array}$} & 0.484 & 0.475 & 0.510 \\
\hline & & & $(0.50)$ & $(0.50)$ & $(0.50)$ \\
\hline & & & 0.487 & 0.487 & 0.485 \\
\hline & Fund(LR) & & $(0.50)$ & $(0.50)$ & $(0.50)$ \\
\hline \multirow{8}{*}{ 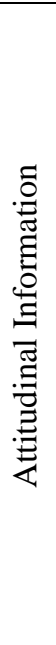 } & & Increased sea level rise is real and we will & 3.626 & 3.545 & 3.848 \\
\hline & $\operatorname{Impact}(\mathrm{SR})$ & $\begin{array}{l}\text { experience impact in the short-term future } \\
(10-20 \text { years })(=1 \text { if strongly disagree, } 5 \text { if } \\
\text { strongly agree })\end{array}$ & $(1.23)$ & $(1.23)$ & $(1.20)$ \\
\hline & & Increased sea level rise is real and we will & 3.751 & 3.680 & 3.949 \\
\hline & $\operatorname{Impact}(\mathrm{LR})$ & $\begin{array}{l}\text { experience impact in the long-term future } \\
(30-50 \text { years })(=1 \text { if strongly disagree, } 5 \text { if } \\
\text { strongly agree) }\end{array}$ & $(1.23)$ & $(1.24)$ & $(1.21)$ \\
\hline & & Increased sea level rise is not real and & 2.171 & 2.142 & 2.253 \\
\hline & $\operatorname{Impact}(\mathrm{No})$ & $\begin{array}{l}\text { there will be no impact (= } 1 \text { if strongly } \\
\text { disagree, } 5 \text { if strongly agree) }\end{array}$ & $(1.26)$ & $(1.20)$ & $(1.41)$ \\
\hline & & Concern about the projected impacts of sea & 2.570 & 2.465 & 2.859 \\
\hline & Concern & $\begin{array}{l}\text { level rise on your well-being. ( }=1 \text { if no } \\
\text { concern, } 4 \text { if high concern) }\end{array}$ & $(0.93)$ & $(0.93)$ & $(0.88)$ \\
\hline \multirow{7}{*}{ 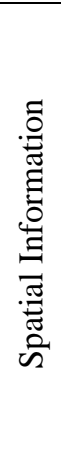 } & Distance & $\begin{array}{l}\text { Log distance from respondents to the } \\
\text { nearest coastline (unit: meter) }\end{array}$ & $\begin{array}{l}9.566 \\
(1.20)\end{array}$ & $\begin{array}{l}9.654 \\
(1.19\end{array}$ & $\begin{array}{l}9.322 \\
(1.20)\end{array}$ \\
\hline & Elayation & Average elevation in the $10 * 10 \mathrm{~km}$ square & 38.887 & 42.220 & 29.629 \\
\hline & Elevation & where respondents fall inside (unit: feet) & $(39.88)$ & $(41.41$ & $(33.67)$ \\
\hline & & The $10 * 10 \mathrm{~km}$ square is expected be & 0.557 & 0.522 & 0.657 \\
\hline & Inund2030 & $\begin{array}{l}\text { inundated by the year of } 2030 . \\
(=1 \text { if yes, } 0 \text { if no) }\end{array}$ & $(0.50)$ & $(0.50)$ & $(0.48)$ \\
\hline & & The $10 * 10 \mathrm{~km}$ square is expected be & 0.584 & 0.547 & 0.687 \\
\hline & Inund2060 & $\begin{array}{l}\text { inundated by the year of } 2060 \text {. } \\
(=1 \text { if yes, } 0 \text { if no) }\end{array}$ & (0.49) & $(0.50)$ & $(0.46)$ \\
\hline
\end{tabular}


Table 2.4: Results of Multinomial Logit Model (Short Term)

\begin{tabular}{|c|c|c|c|c|c|c|}
\hline & Model 1 & Model 2 & Model 3 & Model 4 & Model 5 & Model 6 \\
\hline \multirow[t]{2}{*}{$\operatorname{ASC}(1,2)$} & $0.697^{*}$ & -1.213 & 1.037 & -0.669 & -1.481 & -1.498 \\
\hline & $(0.40)$ & $(0.91)$ & (1.27) & $(0.95)$ & $(0.93)$ & $(0.93)$ \\
\hline \multirow[t]{2}{*}{ Land } & $0.041^{* *}$ & $0.045^{* *}$ & $0.043^{* *}$ & $0.043^{* *}$ & $0.044^{* *}$ & $0.044^{* *}$ \\
\hline & $(0.02)$ & $(0.02)$ & $(0.02)$ & $(0.02)$ & $(0.02)$ & $(0.02)$ \\
\hline \multirow[t]{2}{*}{ Drainage } & $0.057^{* * *}$ & $0.059^{* * *}$ & $0.060^{* * *}$ & $0.052^{* * *}$ & $0.056^{* * *}$ & $0.056^{* * * *}$ \\
\hline & $(0.02)$ & $(0.02)$ & $(0.02)$ & $(0.02)$ & $(0.02)$ & $(0.02)$ \\
\hline \multirow[t]{2}{*}{ Pump } & $0.034^{* *}$ & $0.043^{* *}$ & $0.042^{* *}$ & $0.034^{*}$ & $0.040^{* *}$ & $0.039^{* *}$ \\
\hline & $(0.02)$ & $(0.02)$ & $(0.02)$ & $(0.02)$ & $(0.02)$ & $(0.02)$ \\
\hline \multirow[t]{2}{*}{ Regulation } & 1.243 & 1.391 & 1.376 & 1.123 & 1.313 & 1.289 \\
\hline & $(0.78)$ & $(0.85)$ & $(0.86)$ & $(0.89)$ & $(0.86)$ & $(0.86)$ \\
\hline \multirow[t]{2}{*}{ Cost } & $-0.058^{* * *}$ & $-0.064^{* * *}$ & $-0.063^{* * *}$ & $-0.060^{* * *}$ & $-0.061^{* * *}$ & $-0.061^{* * *}$ \\
\hline & $(0.01)$ & $(0.01)$ & $(0.01)$ & $(0.01)$ & $(0.01)$ & $(0.01)$ \\
\hline \multirow[t]{2}{*}{ ASC $*$ Fund(SR) } & & $0.798^{* * *}$ & $0.860^{* * *}$ & $0.903^{* * *}$ & $0.875^{* * *}$ & $0.864^{* * * *}$ \\
\hline & & $(0.19)$ & $(0.20)$ & $(0.20)$ & $(0.20)$ & $(0.20)$ \\
\hline \multirow[t]{2}{*}{ ASC*Impact(SR) } & & $0.385^{* * *}$ & $0.371^{* * * *}$ & $0.403^{* * *}$ & $0.368^{* * *}$ & $0.368^{* * * *}$ \\
\hline & & $(0.10)$ & $(0.10)$ & $(0.10)$ & $(0.10)$ & $(0.10)$ \\
\hline \multirow[t]{2}{*}{ ASC*Impact(No) } & & $-0.208^{* *}$ & $-0.202^{* *}$ & $-0.184^{* *}$ & $-0.216^{* *}$ & $-0.220^{* *}$ \\
\hline & & $(0.09)$ & $(0.09)$ & $(0.09)$ & $(0.09)$ & $(0.09)$ \\
\hline \multirow[t]{2}{*}{ ASC $*$ Concern } & & $0.272^{* *}$ & $0.249^{* *}$ & $0.203^{*}$ & $0.241^{* *}$ & $0.245^{* *}$ \\
\hline & & $(0.12)$ & $(0.12)$ & $(0.12)$ & $(0.12)$ & $(0.12)$ \\
\hline \multirow[t]{2}{*}{ ASC*Age } & & -0.003 & -0.004 & -0.003 & -0.004 & -0.004 \\
\hline & & $(0.01)$ & $(0.01)$ & $(0.01)$ & $(0.01)$ & $(0.01)$ \\
\hline \multirow[t]{2}{*}{ ASC*Education } & & 0.031 & 0.018 & 0.032 & 0.035 & 0.039 \\
\hline & & $(0.11)$ & $(0.11)$ & $(0.12)$ & $(0.11)$ & $(0.11)$ \\
\hline \multirow[t]{2}{*}{ ASC*Income } & & $0.051^{* *}$ & $0.050^{* *}$ & $0.051^{* *}$ & $0.051^{* *}$ & $0.051^{* *}$ \\
\hline & & $(0.02)$ & $(0.02)$ & $(0.03)$ & $(0.02)$ & $(0.02)$ \\
\hline \multirow[t]{2}{*}{ ASC*Employed } & & 0.345 & 0.320 & 0.227 & 0.290 & 0.285 \\
\hline & & $(0.22)$ & $(0.22)$ & $(0.23)$ & $(0.23)$ & $(0.23)$ \\
\hline \multirow[t]{2}{*}{ ASC ${ }^{*}$ Children } & & -0.088 & -0.076 & -0.036 & -0.071 & -0.078 \\
\hline & & $(0.12)$ & $(0.12)$ & $(0.12)$ & $(0.12)$ & $(0.12)$ \\
\hline \multirow[t]{2}{*}{ ASC*Distance } & & & $-0.227^{* *}$ & & & \\
\hline & & & $(0.09)$ & & & \\
\hline ASC*Elevation & & & & $\begin{array}{c}-0.015 \\
(0.00)\end{array}$ & & \\
\hline \multirow[t]{2}{*}{ ASC*Inund 2030} & & & & & $0.681^{* * *}$ & \\
\hline & & & & & $(0.20)$ & \\
\hline ASC*Inund 2060 & & & & & & $\begin{array}{l}0.676^{* * *} \\
(0.19)\end{array}$ \\
\hline \multirow[t]{2}{*}{ ASC*Yearlong } & & $-0.584^{* *}$ & $-0.521^{* *}$ & -0.431 & $-0.503^{*}$ & $-0.499^{*}$ \\
\hline & & $(0.26)$ & $(0.26)$ & $(0.27)$ & $(0.26)$ & $(0.26)$ \\
\hline Observations & 2244 & 2244 & 2244 & 2244 & 2244 & 2244 \\
\hline Pseudo-R2 & 0.046 & 0.123 & 0.127 & 0.149 & 0.131 & 0.131 \\
\hline Wald chi2 & 75.008 & 202.515 & 209.239 & 244.198 & 214.819 & 214.677 \\
\hline log-likelihood & -784.258 & -720.504 & -717.143 & -699.663 & -714.352 & -714.424 \\
\hline
\end{tabular}


Table 2.5: Results Between Yearlong and Seasonal Residents (Short Term)

\begin{tabular}{|c|c|c|c|c|c|c|}
\hline & \multicolumn{2}{|c|}{ Model 7} & \multicolumn{2}{|c|}{ Model 8} & \multicolumn{2}{|c|}{ Model 9} \\
\hline & Yearlong & Seasonal & Yearlong & Seasonal & Yearlong & Seasonal \\
\hline $\operatorname{ASC}(1,2)$ & $\begin{array}{l}0.146 \\
(045)\end{array}$ & $\begin{array}{c}2.936^{* * *} \\
(098)\end{array}$ & $\begin{array}{c}-2.108^{* * *} \\
(0.98)\end{array}$ & -0.089 & 0.066 & $\begin{array}{l}4.229 \\
(327)\end{array}$ \\
\hline Land & $0.033^{*}$ & $0.089^{* *}$ & $0.034^{*}$ & $\begin{array}{l}0.095^{*} \\
(0.05)\end{array}$ & 0.033 & 0.084 \\
\hline Drainage & $\begin{array}{c}(0.02) \\
0.062^{* * *} \\
(0.02)\end{array}$ & $\begin{array}{l}(0.04) \\
0.050 \\
(0.04)\end{array}$ & $\begin{array}{c}(0.02) \\
0.059^{* * *} \\
(0.02)\end{array}$ & $\begin{array}{c}(0.05) \\
0.119^{* *} \\
(0.06)\end{array}$ & $\begin{array}{c}(0.02) \\
0.060^{* * * *} \\
(0.02)\end{array}$ & $\begin{array}{c}(0.06) \\
0.121^{* *} \\
(0.06)\end{array}$ \\
\hline Pump & $\begin{array}{l}0.016 \\
(0.02)\end{array}$ & $\begin{array}{c}0.115^{* * *} \\
(0.04)\end{array}$ & $\begin{array}{l}0.022 \\
(0.02)\end{array}$ & $\begin{array}{c}0.152^{* * *} \\
(0.06)\end{array}$ & $\begin{array}{l}0.020 \\
(0.02)\end{array}$ & $\begin{array}{c}0.145^{* *} \\
(0.06)\end{array}$ \\
\hline Regulation & $\begin{array}{l}0.770 \\
(0.85)\end{array}$ & $\begin{array}{l}4.031^{*} \\
(2.10)\end{array}$ & $\begin{array}{l}0.633 \\
(0.91)\end{array}$ & $\begin{array}{c}6.467^{* *} \\
(2.90)\end{array}$ & $\begin{array}{l}0.636 \\
(0.92)\end{array}$ & $\begin{array}{c}6.131^{* * *} \\
(3.01)\end{array}$ \\
\hline Cost & $\begin{array}{c}-0.054^{* * * *} \\
(0.01)\end{array}$ & $\begin{array}{c}-0.069^{* * *} \\
(0.02)\end{array}$ & $\begin{array}{c}-0.062^{* * * *} \\
(0.01)\end{array}$ & $\begin{array}{c}-0.074^{* * * *} \\
(0.02)\end{array}$ & $\begin{array}{c}-0.060^{* * * *} \\
(0.01)\end{array}$ & $\begin{array}{c}-0.073^{* * * *} \\
(0.02)\end{array}$ \\
\hline ASC*Fund(SR) & & & $\begin{array}{c}0.777^{* * * *} \\
(0.21)\end{array}$ & $\begin{array}{c}1.255^{* * *} \\
(0.58)\end{array}$ & $\begin{array}{c}0.832^{* * * *} \\
(0.21)\end{array}$ & $\begin{array}{c}1.467^{* * *} \\
(0.62)\end{array}$ \\
\hline ASC*Impact(SR) & & & $\begin{array}{c}0.353^{* * * *} \\
(0.11)\end{array}$ & $\begin{array}{c}0.555^{* * *} \\
(0.25)\end{array}$ & $\begin{array}{c}0.340^{* * * * *} \\
(0.11)\end{array}$ & $\begin{array}{c}0.528^{* * *} \\
(0.25)\end{array}$ \\
\hline ASC*Impact(No) & & & $\begin{array}{c}-0.243^{* *} \\
(0.11)\end{array}$ & $\begin{array}{l}-0.186 \\
(0.20)\end{array}$ & $\begin{array}{c}-0.243^{* *} \\
(0.11)\end{array}$ & $\begin{array}{l}-0.138 \\
(0.21)\end{array}$ \\
\hline ASC*Concern & & & $\begin{array}{l}0.210^{*} \\
(0.13)\end{array}$ & $\begin{array}{l}0.491 \\
(0.36)\end{array}$ & $\begin{array}{l}0.174 \\
(0.13)\end{array}$ & $\begin{array}{l}0.509 \\
(0.38)\end{array}$ \\
\hline ASC*Age & & & $\begin{array}{l}0.006 \\
(0.01)\end{array}$ & $\begin{array}{c}-0.047^{* *} \\
(0.02)\end{array}$ & $\begin{array}{l}0.005 \\
(0.01)\end{array}$ & $\begin{array}{c}-0.052^{* *} \\
(0.02)\end{array}$ \\
\hline ASC*Education & & & $\begin{array}{l}-0.004 \\
(0.12)\end{array}$ & $\begin{array}{l}0.272 \\
(0.32)\end{array}$ & $\begin{array}{l}-0.026 \\
(0.12)\end{array}$ & $\begin{array}{l}0.378 \\
(0.34)\end{array}$ \\
\hline ASC*Income & & & $\begin{array}{l}0.036 \\
(0.03)\end{array}$ & $\begin{array}{l}0.107 \\
(0.07)\end{array}$ & $\begin{array}{l}0.036 \\
(0.03)\end{array}$ & $\begin{array}{l}0.109 \\
(0.07)\end{array}$ \\
\hline ASC*Employed & & & $\begin{array}{c}0.517^{* *} \\
(0.24)\end{array}$ & $\begin{array}{r}-0.767 \\
(0.66)\end{array}$ & $\begin{array}{c}0.482^{* *} \\
(0.25)\end{array}$ & $\begin{array}{l}-0.852 \\
(0.70)\end{array}$ \\
\hline ASC ${ }^{*}$ Children & & & $\begin{array}{l}-0.107 \\
(0.13)\end{array}$ & $\begin{array}{l}-0.108 \\
(0.32)\end{array}$ & $\begin{array}{l}-0.104 \\
(0.13)\end{array}$ & $\begin{array}{l}-0.060 \\
(0.31)\end{array}$ \\
\hline ASC*Distance & & & & & $\begin{array}{c}-0.208^{* * *} \\
(0.10) \\
\end{array}$ & $\begin{array}{c}-0.505^{* *} \\
(0.25) \\
\end{array}$ \\
\hline Observations & 1650 & 594 & 1650 & 594 & 1650 & 594 \\
\hline Pseudo-R2 & 0.035 & 0.124 & 0.102 & 0.229 & 0.106 & 0.239 \\
\hline Wald chi 2 & 42.560 & 54.084 & 123.058 & 99.625 & 127.746 & 104.035 \\
\hline log-likelihood & -582.957 & -190.483 & -542.708 & -167.713 & -540.364 & -165.508 \\
\hline
\end{tabular}


Table 2.6: Results of Multinomial Logit Model (Long Term)

\begin{tabular}{|c|c|c|c|c|c|c|}
\hline & Model 1 & Model 2 & Model 3 & Model 4 & Model 5 & Model 6 \\
\hline $\operatorname{ASC}(1,2)$ & $\begin{array}{c}-1.211^{* * * *} \\
(0.40)\end{array}$ & $\begin{array}{c}-2.105^{* *} \\
(0.95)\end{array}$ & $\begin{array}{l}0.681 \\
(1.27)\end{array}$ & $\begin{array}{c}-1.756^{*} \\
(0.96)\end{array}$ & $\begin{array}{c}-2.178^{* *} \\
(0.95)\end{array}$ & $\begin{array}{c}-2.210^{* * *} \\
(0.95)\end{array}$ \\
\hline Wetland & $\begin{array}{c}0.082^{* * * *} \\
(0.02)\end{array}$ & $\begin{array}{c}0.087^{* * * *} \\
(0.02)\end{array}$ & $\begin{array}{c}0.091^{* * * *} \\
(0.02)\end{array}$ & $\begin{array}{c}0.084^{* * * *} \\
(0.02)\end{array}$ & $\begin{array}{c}0.086^{* * * *} \\
(0.02)\end{array}$ & $\begin{array}{c}0.086^{* * * *} \\
(0.02)\end{array}$ \\
\hline Flood & $\begin{array}{c}0.074^{* * * *} \\
(0.02)\end{array}$ & $\begin{array}{c}0.085^{* * * *} \\
(0.02)\end{array}$ & $\begin{array}{c}0.086^{* * * *} \\
(0.02)\end{array}$ & $\begin{array}{c}0.088^{* * * *} \\
(0.02)\end{array}$ & $\begin{array}{c}0.084^{* * * *} \\
(0.02)\end{array}$ & $\begin{array}{c}0.084^{* * * *} \\
(0.02)\end{array}$ \\
\hline Regulation & $\begin{array}{l}1.224^{*} \\
(0.71)\end{array}$ & $\begin{array}{l}1.396^{*} \\
(0.77)\end{array}$ & $\begin{array}{l}1.511^{*} \\
(0.78)\end{array}$ & $\begin{array}{l}1.387^{*} \\
(0.78)\end{array}$ & $\begin{array}{l}1.339^{*} \\
(0.77)\end{array}$ & $\begin{array}{l}1.364^{*} \\
(0.77)\end{array}$ \\
\hline Cost & $\begin{array}{c}-0.054^{* * * *} \\
(0.01)\end{array}$ & $\begin{array}{c}-0.057^{* * * *} \\
(0.01)\end{array}$ & $\begin{array}{c}-0.057^{* * * *} \\
(0.01)\end{array}$ & $\begin{array}{c}-0.057^{* * * *} \\
(0.01)\end{array}$ & $\begin{array}{c}-0.057^{* * * *} \\
(0.01)\end{array}$ & $\begin{array}{c}-0.057^{* * * *} \\
(0.01)\end{array}$ \\
\hline ASC*Fund(LR) & & $\begin{array}{l}-0.041 \\
(0.19)\end{array}$ & $\begin{array}{l}-0.033 \\
(0.19)\end{array}$ & $\begin{array}{l}-0.061 \\
(0.19)\end{array}$ & $\begin{array}{l}-0.041 \\
(0.19)\end{array}$ & $\begin{array}{l}-0.037 \\
(0.19)\end{array}$ \\
\hline ASC*Impact(LR) & & $\begin{array}{l}0.178^{*} \\
(0.09)\end{array}$ & $\begin{array}{c}0.183^{* *} \\
(0.09)\end{array}$ & $\begin{array}{c}0.189^{* * *} \\
(0.09)\end{array}$ & $\begin{array}{c}0.179^{* * *} \\
(0.09)\end{array}$ & $\begin{array}{c}0.179^{* *} \\
(0.09)\end{array}$ \\
\hline ASC*Impact(No) & & $\begin{array}{c}-0.337^{* * *} \\
(0.09)\end{array}$ & $\begin{array}{c}-0.317^{* * *} \\
(0.09)\end{array}$ & $\begin{array}{c}-0.320^{* * *} \\
(0.09)\end{array}$ & $\begin{array}{c}-0.334^{* * * *} \\
(0.09)\end{array}$ & $\begin{array}{c}-0.336^{* * * *} \\
(0.09)\end{array}$ \\
\hline ASC $*$ Concern & & $\begin{array}{c}0.448^{* * *} \\
(0.11)\end{array}$ & $\begin{array}{c}0.421^{* * * *} \\
(0.11)\end{array}$ & $\begin{array}{c}0.404^{* * * *} \\
(0.11)\end{array}$ & $\begin{array}{c}0.429^{* * * *} \\
(0.11)\end{array}$ & $\begin{array}{c}0.427^{* * * *} \\
(0.11)\end{array}$ \\
\hline ASC*Age & & $\begin{array}{l}0.001 \\
(0.01)\end{array}$ & $\begin{array}{l}0.001 \\
(0.01)\end{array}$ & $\begin{array}{l}0.002 \\
(0.01)\end{array}$ & $\begin{array}{l}0.001 \\
(0.01)\end{array}$ & $\begin{array}{l}0.001 \\
(0.01)\end{array}$ \\
\hline ASC*Education & & $\begin{array}{l}-0.151 \\
(0.11)\end{array}$ & $\begin{array}{c}-0.181 \\
(0.12)\end{array}$ & $\begin{array}{l}-0.158 \\
(0.11)\end{array}$ & $\begin{array}{r}-0.155 \\
(0.11)\end{array}$ & $\begin{array}{l}-0.154 \\
(0.11)\end{array}$ \\
\hline ASC*Income & & $\begin{array}{c}0.050^{* * *} \\
(0.02)\end{array}$ & $\begin{array}{c}0.050^{* * *} \\
(0.02)\end{array}$ & $\begin{array}{c}0.050^{* * *} \\
(0.02)\end{array}$ & $\begin{array}{c}0.049^{* * *} \\
(0.02)\end{array}$ & $\begin{array}{c}0.049^{* *} \\
(0.02)\end{array}$ \\
\hline ASC*Employed & & $\begin{array}{l}0.198 \\
(0.22)\end{array}$ & $\begin{array}{l}0.192 \\
(0.22)\end{array}$ & $\begin{array}{l}0.144 \\
(0.22)\end{array}$ & $\begin{array}{l}0.186 \\
(0.22)\end{array}$ & $\begin{array}{l}0.180 \\
(0.22)\end{array}$ \\
\hline ASC $*$ Children & & $\begin{array}{l}0.002 \\
(0.11)\end{array}$ & $\begin{array}{l}0.014 \\
(0.12)\end{array}$ & $\begin{array}{l}0.021 \\
(0.12)\end{array}$ & $\begin{array}{l}0.011 \\
(0.12)\end{array}$ & $\begin{array}{l}0.008 \\
(0.12)\end{array}$ \\
\hline ASC*Distance & & & $\begin{array}{c}-0.288^{* * * *} \\
(0.09)\end{array}$ & & & \\
\hline ASC*Elevation & & & & $\begin{array}{c}-0.008^{* * *} \\
(0.00)\end{array}$ & & \\
\hline ASC*Inund2030 & & & & & $\begin{array}{l}0.290 \\
(0.19)\end{array}$ & \\
\hline ASC*Inund2060 & & & & & & $\begin{array}{l}0.339^{*} \\
(0.19)\end{array}$ \\
\hline ASC*Yearlong & & $\begin{array}{c}-0.780^{* * *} \\
(0.25)\end{array}$ & $\begin{array}{c}-0.723^{* * *} \\
(0.26)\end{array}$ & $\begin{array}{c}-0.686^{* * * *} \\
(0.26)\end{array}$ & $\begin{array}{c}-0.758^{* * * *} \\
(0.26)\end{array}$ & $\begin{array}{c}-0.748^{* * *} \\
(0.26)\end{array}$ \\
\hline Observations & 2244 & 2244 & 2244 & 2244 & 2244 & 2244 \\
\hline Pseudo-R2 & 0.070 & 0.133 & 0.140 & 0.140 & 0.134 & 0.135 \\
\hline Wald chi2 & 114.810 & 218.161 & 229.473 & 230.682 & 220.508 & 221.375 \\
\hline log-likelihood & -764.357 & -712.682 & -707.025 & -706.421 & -711.508 & -711.074 \\
\hline
\end{tabular}


Table 2.7: Results Between Yearlong and Seasonal Residents (Long Term)

\begin{tabular}{|c|c|c|c|c|c|c|}
\hline & \multicolumn{2}{|c|}{ Model 7} & \multicolumn{2}{|c|}{ Model 8} & \multicolumn{2}{|c|}{ Model 9} \\
\hline & Yearlong & Seasonal & Yearlong & Seasonal & Yearlong & Seasonal \\
\hline $\operatorname{ASC}(1,2)$ & $\begin{array}{c}-1.081^{* * *} \\
(0.46)\end{array}$ & $\begin{array}{c}-1.667^{*} \\
(0.89)\end{array}$ & $\begin{array}{c}-2.350^{* *} \\
(1.02)\end{array}$ & $\begin{array}{c}-3.942^{*} \\
(2.24)\end{array}$ & $\begin{array}{l}0.780 \\
(1.42)\end{array}$ & $\begin{array}{l}-1.815 \\
(2.96)\end{array}$ \\
\hline Wetland & $\begin{array}{c}0.083^{* * *} \\
(0.02)\end{array}$ & $\begin{array}{c}0.091^{* *} \\
(0.04)\end{array}$ & $\begin{array}{c}0.083^{\text {**** }} \\
(0.02)\end{array}$ & $\begin{array}{c}0.127^{\text {**** }} \\
(0.05)\end{array}$ & $\begin{array}{c}0.089^{* * *} \\
(0.02)\end{array}$ & $\begin{array}{c}0.124^{* *} \\
(0.05)\end{array}$ \\
\hline Flood & $\begin{array}{c}0.058^{* * * *} \\
(0.02)\end{array}$ & $\begin{array}{c}0.129^{* * *} \\
(0.04)\end{array}$ & $\begin{array}{c}0.071^{\text {**** }} \\
(0.02)\end{array}$ & $\begin{array}{c}0.153^{\text {**** }} \\
(0.05)\end{array}$ & $\begin{array}{c}0.072^{* * *} \\
(0.02)\end{array}$ & $\begin{array}{c}0.153^{* * *} \\
(0.05)\end{array}$ \\
\hline Regulation & $\begin{array}{l}0.602 \\
(0.81)\end{array}$ & $\begin{array}{l}2.906^{*} \\
(1.58)\end{array}$ & $\begin{array}{l}0.657 \\
(0.87)\end{array}$ & $\begin{array}{c}4.211^{* *} \\
(1.77)\end{array}$ & $\begin{array}{c}0.797 \\
(0.88)\end{array}$ & $\begin{array}{c}4.184^{* *} \\
(1.77)\end{array}$ \\
\hline Cost & $\begin{array}{c}-0.062^{* * * *} \\
(0.01)\end{array}$ & $\begin{array}{c}-0.041^{*} \\
(0.02)\end{array}$ & $\begin{array}{c}-0.067^{* * *} \\
(0.01)\end{array}$ & $\begin{array}{c}-0.039^{*} \\
(0.02)\end{array}$ & $\begin{array}{c}-0.067^{* * *} \\
(0.01)\end{array}$ & $\begin{array}{c}-0.038^{*} \\
(0.02)\end{array}$ \\
\hline ASC $*$ Fund(LR) & & & $\begin{array}{l}0.099 \\
(0.21)\end{array}$ & $\begin{array}{l}-0.779 \\
(0.48)\end{array}$ & $\begin{array}{l}0.087 \\
(0.21)\end{array}$ & $\begin{array}{c}-0.726 \\
(0.49)\end{array}$ \\
\hline ASC*Impact(LR) & & & $\begin{array}{l}0.125 \\
(0.11)\end{array}$ & $\begin{array}{l}0.350 \\
(0.22)\end{array}$ & $\begin{array}{l}0.126 \\
(0.11)\end{array}$ & $\begin{array}{l}0.346 \\
(0.22)\end{array}$ \\
\hline $\mathrm{ASC} * \operatorname{Impact}(\mathrm{No})$ & & & $\begin{array}{c}-0.417^{* * * *} \\
(0.10)\end{array}$ & $\begin{array}{l}-0.187 \\
(0.19)\end{array}$ & $\begin{array}{c}-0.405^{* * *} \\
(0.11)\end{array}$ & $\begin{array}{c}-0.166 \\
(0.19)\end{array}$ \\
\hline ASC $*$ Concern & & & $\begin{array}{c}0.376^{* * * *} \\
(0.12)\end{array}$ & $\begin{array}{c}0.785^{* *} \\
(0.31)\end{array}$ & $\begin{array}{c}0.327^{* * *} \\
(0.13)\end{array}$ & $\begin{array}{c}0.782^{* *} \\
(0.31)\end{array}$ \\
\hline ASC*Age & & & $\begin{array}{l}0.006 \\
(0.01)\end{array}$ & $\begin{array}{l}-0.013 \\
(0.02)\end{array}$ & $\begin{array}{l}0.006 \\
(0.01)\end{array}$ & $\begin{array}{l}-0.015 \\
(0.02)\end{array}$ \\
\hline ASC*Education & & & $\begin{array}{l}-0.140 \\
(0.12)\end{array}$ & $\begin{array}{l}-0.108 \\
(0.33)\end{array}$ & $\begin{array}{l}-0.180 \\
(0.12)\end{array}$ & $\begin{array}{c}-0.078 \\
(0.33)\end{array}$ \\
\hline ASC*Income & & & $\begin{array}{l}0.047^{*} \\
(0.03)\end{array}$ & $\begin{array}{l}0.025 \\
(0.06)\end{array}$ & $\begin{array}{l}0.048^{*} \\
(0.03)\end{array}$ & $\begin{array}{l}0.019 \\
(0.06)\end{array}$ \\
\hline ASC*Employed & & & $\begin{array}{l}0.323 \\
(0.24)\end{array}$ & $\begin{array}{l}-0.596 \\
(0.59)\end{array}$ & $\begin{array}{l}0.288 \\
(0.25)\end{array}$ & $\begin{array}{l}-0.563 \\
(0.60)\end{array}$ \\
\hline ASC*Children & & & $\begin{array}{l}-0.002 \\
(0.13)\end{array}$ & $\begin{array}{l}-0.004 \\
(0.27)\end{array}$ & $\begin{array}{l}-0.001 \\
(0.13)\end{array}$ & $\begin{array}{l}0.001 \\
(0.26)\end{array}$ \\
\hline ASC*Distance & & & & & $\begin{array}{c}-0.308^{* * *} \\
(0.10)\end{array}$ & $\begin{array}{l}-0.224 \\
(0.20) \\
\end{array}$ \\
\hline Observations & 1650 & 594 & 1650 & 594 & 1650 & 594 \\
\hline Pseudo-R2 & 0.069 & 0.120 & 0.126 & 0.182 & 0.135 & 0.185 \\
\hline Wald chi2 & 83.951 & 52.319 & 152.426 & 79.316 & 162.748 & 80.568 \\
\hline log-likelihood & -562.261 & -191.366 & -528.024 & -177.867 & -522.863 & -177.241 \\
\hline
\end{tabular}


Table 2.8: MWTP for Attributes in the Short and Long Term

\begin{tabular}{|c|c|c|c|c|c|c|c|}
\hline & \multirow[b]{2}{*}{ Attribute } & \multicolumn{2}{|c|}{ Combined } & \multicolumn{2}{|c|}{ Yearlong } & \multicolumn{2}{|c|}{ Seasonal } \\
\hline & & Basic & Extended & Basic & Extended & Basic & Extended \\
\hline \multirow{3}{*}{$\begin{array}{l}\text { Short } \\
\text { Term }\end{array}$} & Land & $\begin{array}{c}71.2 \\
(9.3,133.1)\end{array}$ & $\begin{array}{c}\mathbf{6 7 . 2} \\
(5.0,129.4)\end{array}$ & $\begin{array}{c}\mathbf{6 0 . 5} \\
(-11.8,132.9)\end{array}$ & $\begin{array}{c}\mathbf{5 4 . 0} \\
(-15.7,123.7)\end{array}$ & $\begin{array}{c}128.4 \\
(-12.1,268.9)\end{array}$ & $\begin{array}{c}116.0 \\
(-58.8,290.8)\end{array}$ \\
\hline & Drainage & $\begin{array}{c}\mathbf{9 8 . 5} \\
(33.7,163.4)\end{array}$ & $\begin{array}{c}95.1 \\
(29.5,160.6)\end{array}$ & $\begin{array}{c}114.0 \\
(34.6,193.5)\end{array}$ & $\begin{array}{c}99.4 \\
(23.2,175.6)\end{array}$ & $\begin{array}{c}72.9 \\
(-53.5,199.3)\end{array}$ & $\begin{array}{c}166.8 \\
(-24.9,358.4)\end{array}$ \\
\hline & Pump & $\begin{array}{c}\mathbf{5 9 . 6} \\
(-1.7,120.8)\end{array}$ & $\begin{array}{c}\mathbf{6 9 . 2} \\
(6.3,132.2)\end{array}$ & $\begin{array}{c}29.2 \\
(-44.8,99.3)\end{array}$ & $\begin{array}{c}32.9 \\
(-36.1,101.8)\end{array}$ & $\begin{array}{c}166.7 \\
(8.3,325.1)\end{array}$ & $\begin{array}{c}\mathbf{2 0 0 . 1} \\
(-3.2,403.3)\end{array}$ \\
\hline Long & Wetland & $\begin{array}{c}\mathbf{1 5 3 . 5} \\
(65.0,242.0)\end{array}$ & $\begin{array}{c}163.8 \\
(68.5,259.0)\end{array}$ & $\begin{array}{c}132.8 \\
(51.6,214.0)\end{array}$ & $\begin{array}{c}133.1 \\
(51.5,214.8)\end{array}$ & $\begin{array}{c}220.5 \\
(-86.0,527.0)\end{array}$ & $\begin{array}{c}\mathbf{3 2 4 . 5} \\
(-137.6,786.7)\end{array}$ \\
\hline Term & Flood & $\begin{array}{c}137.7 \\
(53.9,221.6)\end{array}$ & $\begin{array}{c}\mathbf{1 6 0 . 6} \\
(66.5,254.8)\end{array}$ & $\begin{array}{c}92.9 \\
(20.2,165.6)\end{array}$ & $\begin{array}{c}106.5 \\
(30.0,183.1)\end{array}$ & $\begin{array}{c}\text { 313.7 } \\
(-63.2,690.7)\end{array}$ & $\begin{array}{c}\mathbf{3 9 8 . 8} \\
(-122.8,920.3)\end{array}$ \\
\hline
\end{tabular}

Note: MWTP estimates in bold; 95\% confidence interval in parentheses. The extended models are calculated based on the distance model (Model 3 in Table 2.4 and Model 8 in Table 2.5 for the short term, Model 3 in Table 2.6 and Model 8 in Table 2.7 for the long term) 


\section{Figures}

Figure 2.1: Impact of SLR on Areas of Florida from Year 2030 to 2010
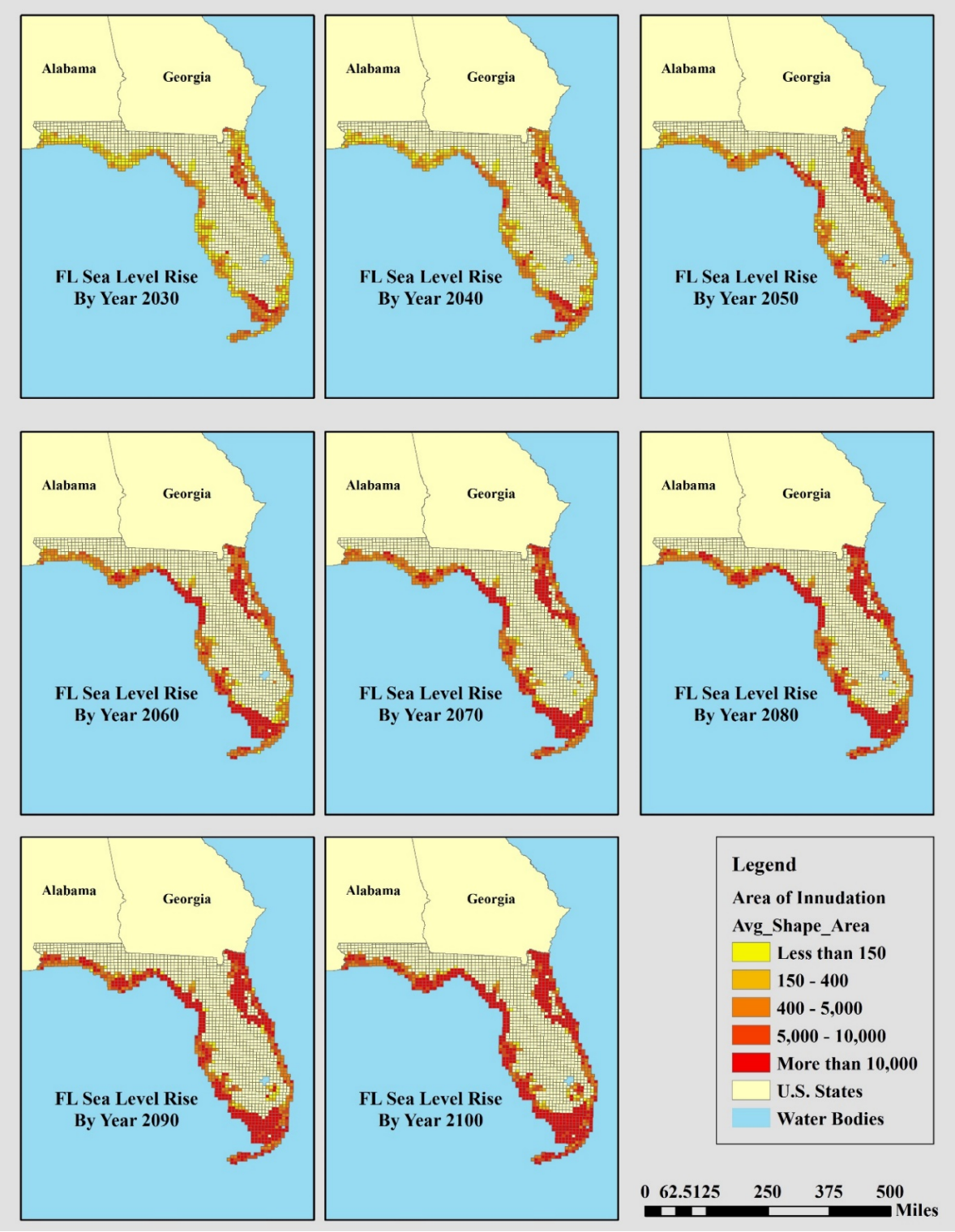
Figure 2.2: Estimated Projection in Florida by USACE

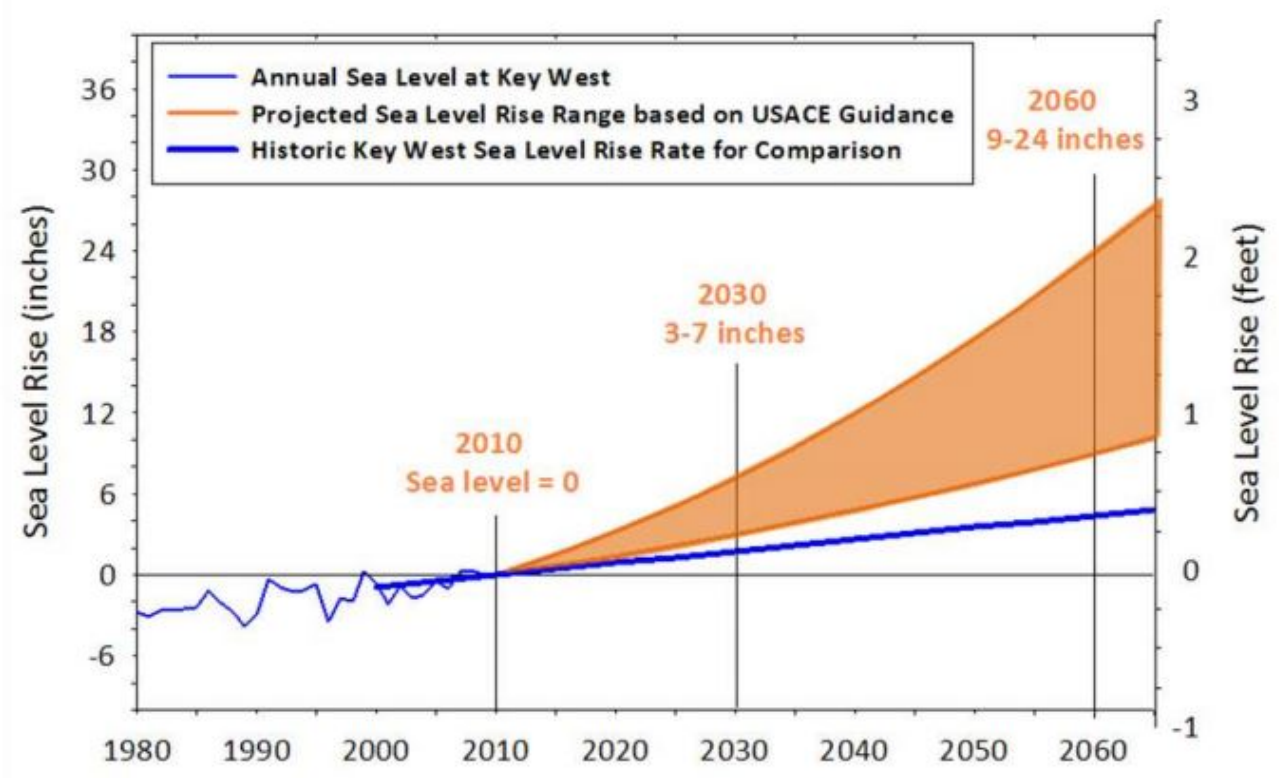

Notes: The projection used historic tidal information from Key West and was calculated by Kristopher Esterson from the United States Army Corps of Engineers, using USACE Guidance (USACE, 2009) intermediate and high curves to represent the lower and upper bound for projected sea level rise in Southeast Florida. Sea level in Florida is projected a rise of three to seven inches by 2030 and nine to twenty-four inches by 2060. See details at: http://www.southeastfloridaclimatecompact.org//wp-content/uploads/2014/09/ sea-level-rise.pdf 
Figure 2.3: Differences in Risk Perception among Yearlong and Seasonal Residents

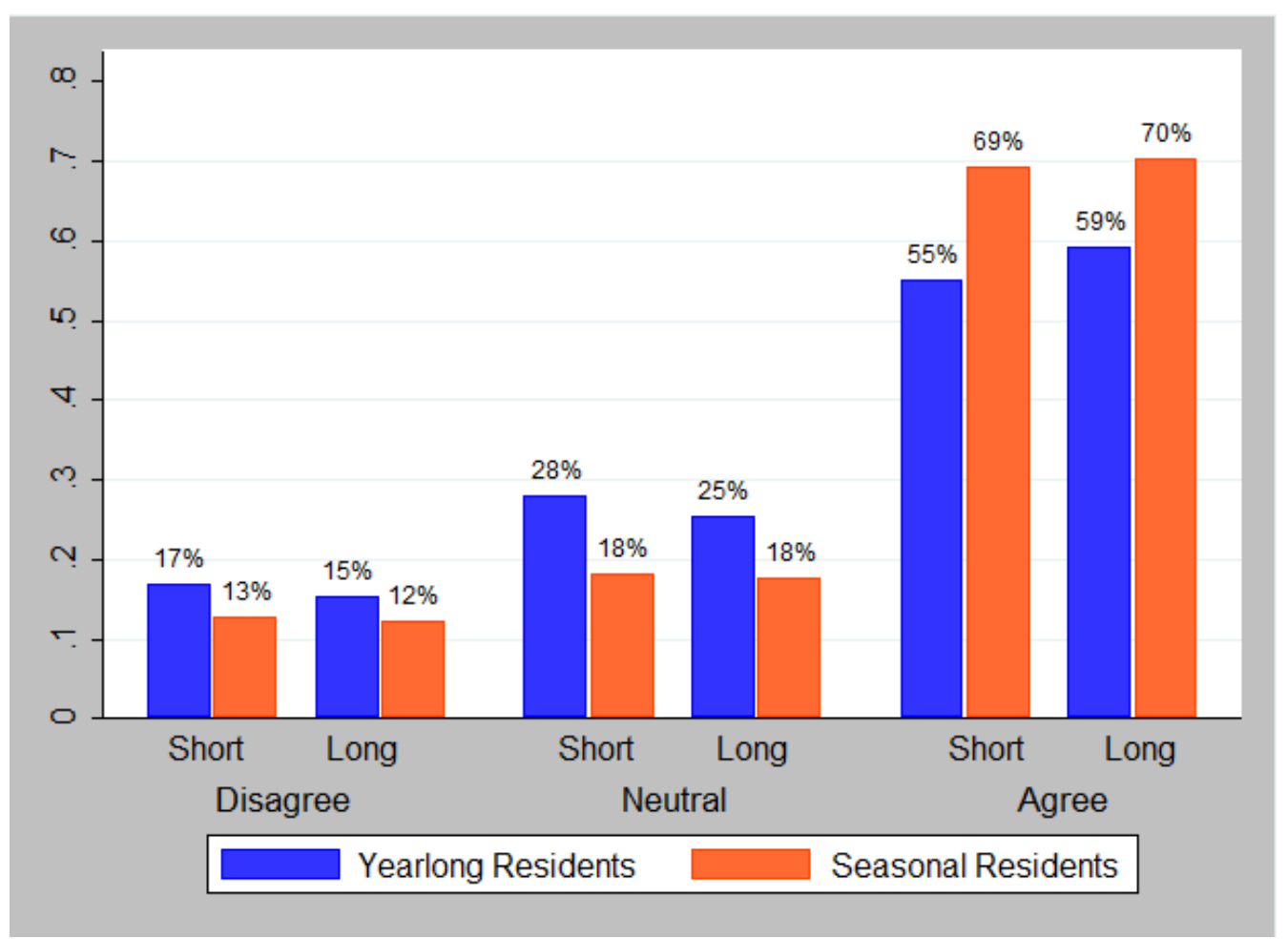

Notes: Respondents were asked about their levels of agreement on the statement that increased sea level rise is real and we will experience impacts in the short term $(10-20$ years) or in the long term (30 - 50 years). 
Figure 2.4: Differences in Concerns among Yearlong and Seasonal Residents

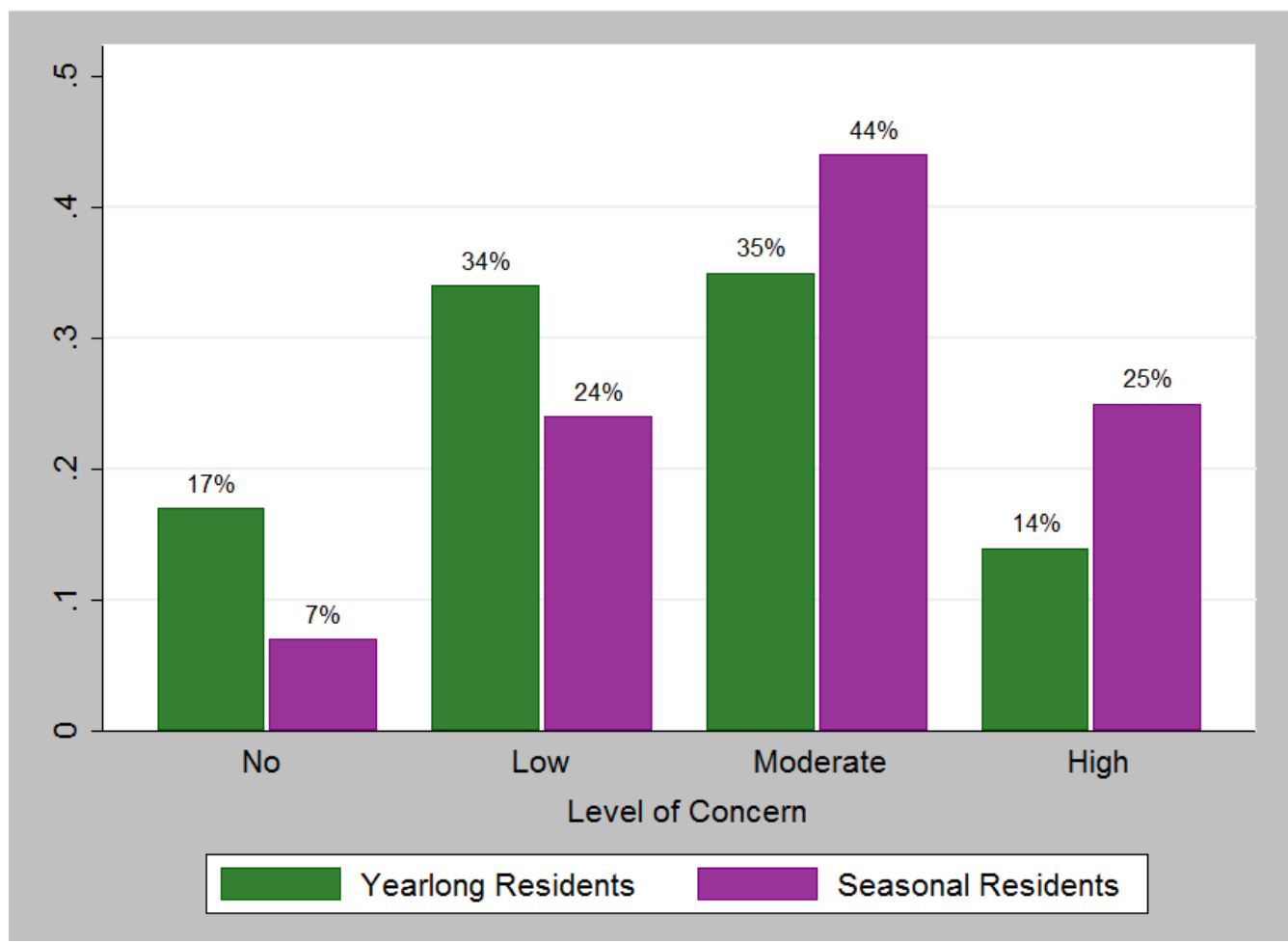

Notes: Respondents were asked about their levels of concern for the projected sea level rise on their well-being (finance, property, health). 
Figure 2.5: Elevation Map of Florida and Locations of Surveyed Respondents

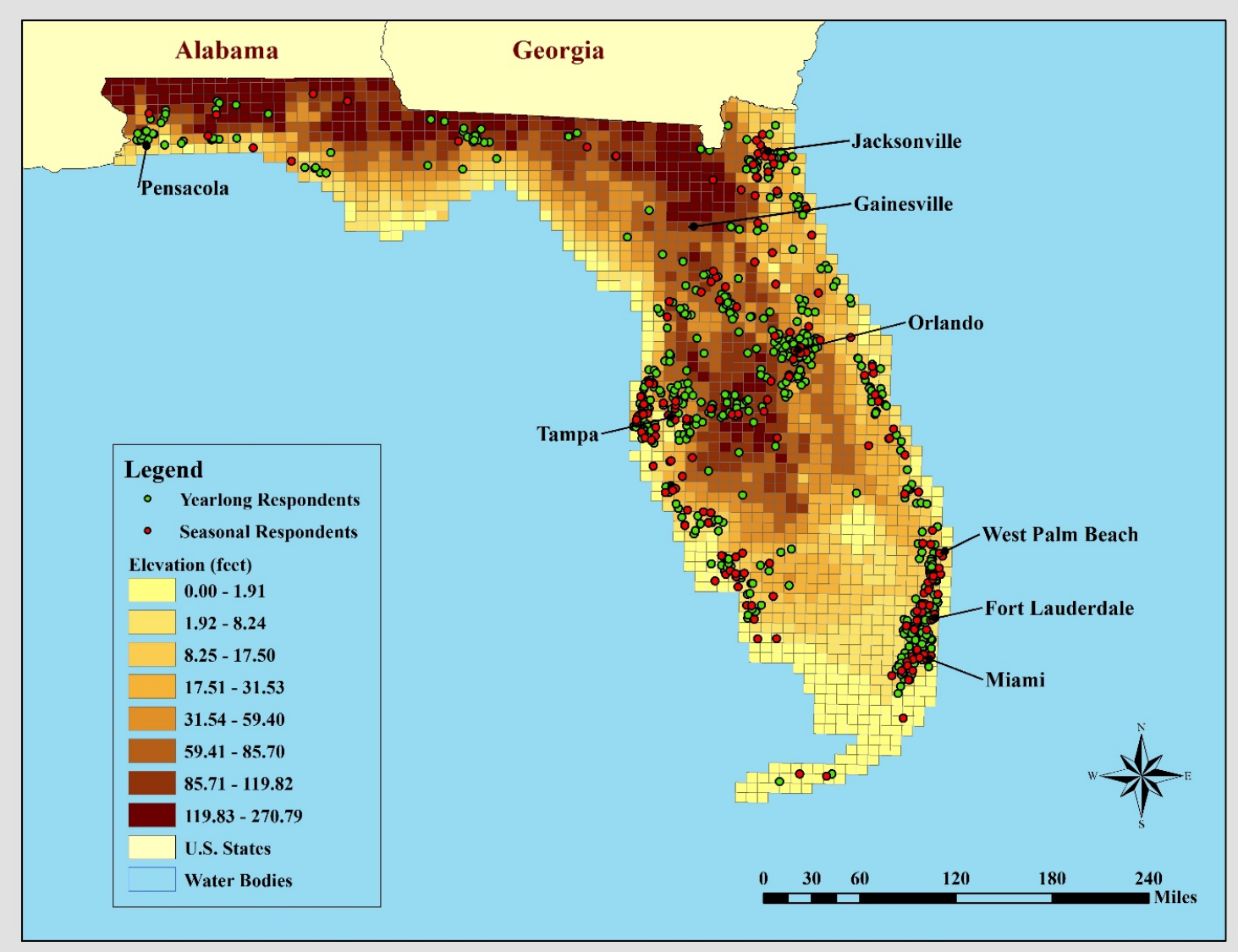


Figure 2.6: Areas of Inundation in Florida and the Affected Respondents

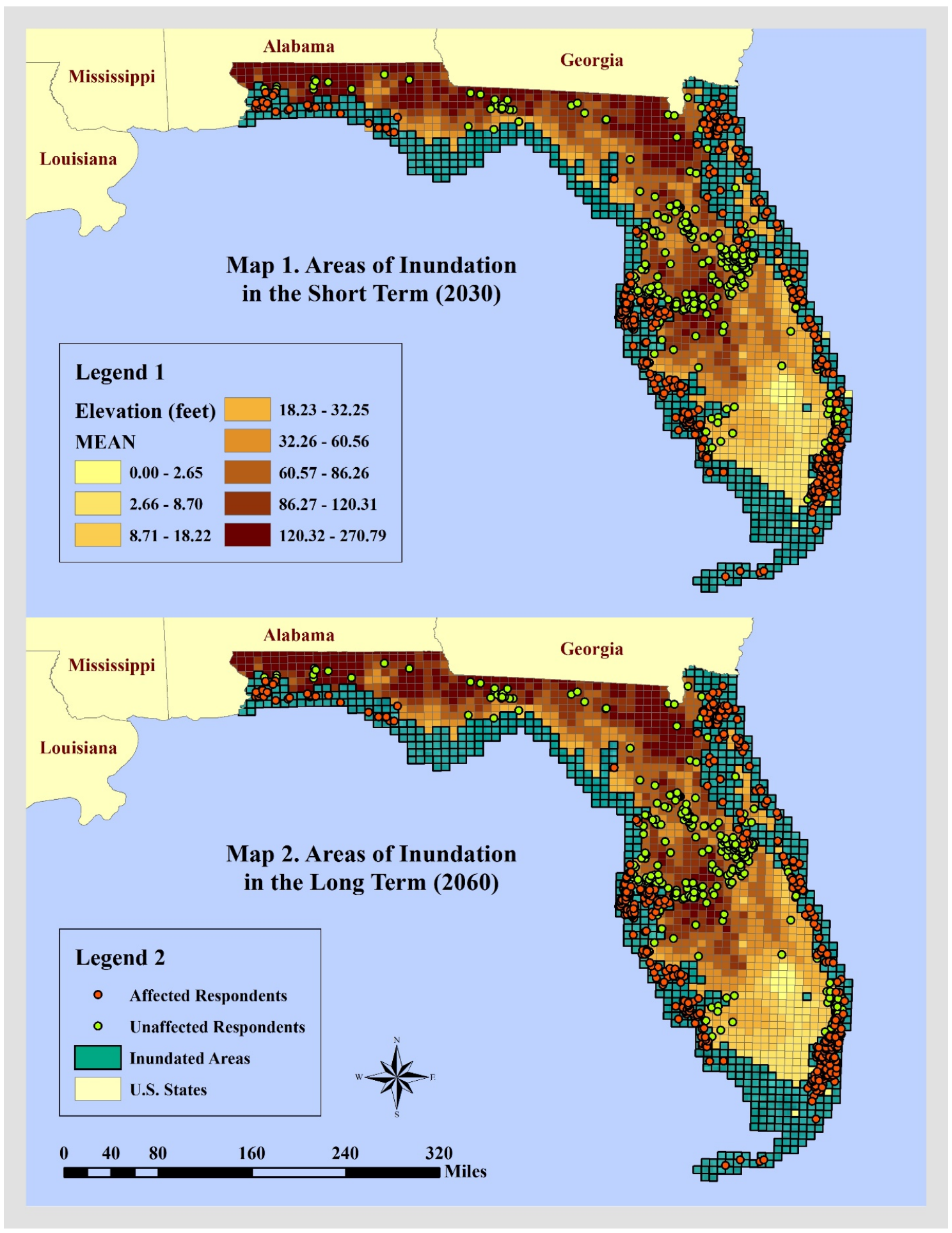




\section{Appendix A. Example of Short Term Choice Card}

The issue of sea level rise is beyond the control of local communities but they can prepare for the impacts through implementing various adaptation strategies. The following question presents several adaptation plans, with various strategies aimed at adapting to increased sea level rise, in the short term (10-20 years) in coastal counties in Florida. You must choose which plan you would prefer most, by considering each of the various plans and the proposed implementation mechanism within each plan. Your payment listed under each plan would be made through an annual local tax over the next 10 years.

\begin{tabular}{|c|c|c|c|}
\hline $\begin{array}{l}\text { Short Term } \\
\text { Adaptation Plan } \\
\text { Components }\end{array}$ & Take No Action & $\begin{array}{l}\text { Short Term } \\
\text { Adaptation Plan A }\end{array}$ & $\begin{array}{l}\text { Short Term } \\
\text { Adaptation Plan B }\end{array}$ \\
\hline $\begin{array}{l}\text { Purchase of } \\
\text { coastally vulnerable } \\
\text { lands and properties } \\
\text { for floodplain } \\
\text { development }\end{array}$ & $\begin{array}{l}\text { No vulnerable } \\
\text { lands or properties } \\
\text { are purchased }\end{array}$ & $\begin{array}{l}10 \% \text { of vulnerable } \\
\text { lands and properties } \\
\text { are purchased; land } \\
\text { is left as a natural } \\
\text { floodplain }\end{array}$ & $\begin{array}{l}30 \% \text { of vulnerable } \\
\text { lands and properties } \\
\text { are purchased: land } \\
\text { is left as a natural } \\
\text { floodplain }\end{array}$ \\
\hline \multirow{2}{*}{$\begin{array}{l}\text { Storm water } \\
\text { management: } \\
\text { relocation of } \\
\text { underground } \\
\text { drainage or, } \\
\text { installation of new } \\
\text { pump station }\end{array}$} & $\begin{array}{l}\text { No- re-plumbing of } \\
\text { low lying drainage }\end{array}$ & $\begin{array}{l}40 \% \text { of low-lying or } \\
\text { underground } \\
\text { drainage is } \\
\text { relocated }\end{array}$ & $\begin{array}{l}60 \text { of low lying or } \\
\text { underground } \\
\text { drainage is } \\
\text { relocated }\end{array}$ \\
\hline & $\begin{array}{l}\text { No new pump } \\
\text { stations are } \\
\text { installed }\end{array}$ & $\begin{array}{l}10 \text { new pump } \\
\text { stations are } \\
\text { installed }\end{array}$ & $\begin{array}{l}40 \text { new pump } \\
\text { stations are } \\
\text { installed }\end{array}$ \\
\hline $\begin{array}{l}\text { New regulation for } \\
\text { raising elevation } \\
\text { requirements for } \\
\text { coastal properties }\end{array}$ & $\begin{array}{l}\text { Areas in buildings } \\
\text { for residential or } \\
\text { commercial use } \\
\text { MUST be } 4 \text { feet } \\
\text { above sea level }\end{array}$ & $\begin{array}{l}\text { Areas in buildings } \\
\text { for residential or } \\
\text { commercial use } \\
\text { MUST be } 3 \text { feet } \\
\text { above sea level }\end{array}$ & $\begin{array}{l}\text { Areas in buildings } \\
\text { for residential or } \\
\text { commercial use } \\
\text { MUST be } 2 \text { feet } \\
\text { above sea level }\end{array}$ \\
\hline $\begin{array}{l}\text { Payment in Annual } \\
\text { Local Taxes }\end{array}$ & $\$ 0$ & $\$ 30$ & $\$ 60$ \\
\hline
\end{tabular}




\section{Appendix B. Example of Long Term Choice Card (with Matching Fund Program)}

As global average temperature continues to rise, sea level rise will continue to accelerate, and its effects will be felt in the long term (30-50 years). As such, the adaptation strategies that worked in the short- term may not be feasible or wise solutions in dealing with sea level rise in the long term. The following question presents several adaptation plans, with various strategies aimed at adapting to increased sea level rise, in the long term (30-50 years) in coastal counties in Florida. You must choose which plan you would prefer most, by considering each of the various plans and the proposed implementation mechanisms within each plan. Your payment listed under each plan would be made through an annual local tax over the next 20 years. Additionally, local tax revenues raised through your personal contribution will be matched $\$ 1$ per $\$ 1$ through funding from the federal government (i.e. for every $\$ 1$ spent on adaptation, the federal government will commit $\$ 1$ towards these adaptation strategies).

\begin{tabular}{|c|c|c|c|}
\hline $\begin{array}{l}\text { Long Term } \\
\text { Adaptation Plan } \\
\text { Components } \\
\end{array}$ & Take No Action & $\begin{array}{l}\text { Long Term } \\
\text { Adaptation Plan A }\end{array}$ & $\begin{array}{l}\text { Long Term } \\
\text { Adaptation Plan B }\end{array}$ \\
\hline $\begin{array}{l}\text { Coastal Wetland } \\
\text { Restoration }\end{array}$ & $\begin{array}{l}\text { No coastal } \\
\text { restoration }\end{array}$ & $\begin{array}{l}30 \% \text { more coastal } \\
\text { restoration }\end{array}$ & $\begin{array}{l}50 \% \text { more coastal } \\
\text { restoration }\end{array}$ \\
\hline $\begin{array}{l}\text { Urban flood } \\
\text { Management }\end{array}$ & $\begin{array}{l}\text { No upgrades to } \\
\text { existing canals for } \\
\text { better drainage; } \\
\text { frequent inland } \\
\text { flooding }\end{array}$ & $\begin{array}{l}\text { Only major canals } \\
\text { are upgraded to } \\
\text { allow for better } \\
\text { drainage; } 40 \% \\
\text { reduction in flooding }\end{array}$ & $\begin{array}{l}\text { All canals are } \\
\text { upgraded within } \\
\text { your county; } 60 \% \\
\text { reduction in } \\
\text { flooding }\end{array}$ \\
\hline $\begin{array}{l}\text { New regulation } \\
\text { for raising } \\
\text { elevation } \\
\text { requirements for } \\
\text { coastal properties }\end{array}$ & $\begin{array}{l}\text { Areas in buildings } \\
\text { for residential or } \\
\text { commercial use } \\
\text { MUST be } 4 \text { feet } \\
\text { above sea level }\end{array}$ & $\begin{array}{l}\text { Areas in buildings } \\
\text { for residential or } \\
\text { commercial use } \\
\text { MUST be } 3 \text { feet } \\
\text { above sea level }\end{array}$ & $\begin{array}{l}\text { Areas in buildings } \\
\text { for residential or } \\
\text { commercial use } \\
\text { MUST be } 2 \text { feet } \\
\text { above sea level }\end{array}$ \\
\hline $\begin{array}{l}\text { Payment in } \\
\text { Annual Taxes }\end{array}$ & $\$ 0$ & $\$ 40$ & $\$ 80$ \\
\hline
\end{tabular}


CHAPTER 3

\section{INSTITUTIONS AND NATURAL DISASTERS: EVIDENCE FROM AGRICULTURAL TRADE IN CENTRAL AMERICA AND THE CARIBBEAN}

\subsection{Introduction}

Natural disasters are events that exceed a community's ability to cope and respond independently (Lindell \& Prater, 2003). No country is immune to nature, but the effects tend to be considerably worse in developing countries which have limited mechanisms to cope with them. In particular, many developing countries, some of which are among the poorest of the poor, are situated in areas prone to natural disasters. This is the case of Haiti or Nicaragua which, for example, in two decades (1980 - 2000) had three major droughts, five floods, and seven hurricanes. Unfortunately, due to global warming, the frequency of natural disasters has been increasing over time and is expected to increase even more.

In these cases, natural disasters often wipe out the whole country's productive infrastructure. The impact is magnified when we consider small developing nations and more specifically, small island nations. Nearly always those countries rely on the agricultural sectors for survival. They export mostly commodities (e.g., fish, bananas, nutmeg, mace, and palm sugar) which account for the largest share of their gross domestic products (GDP), showing a huge dependence on the agricultural industry. Furthermore, these countries are so poorly diversified that any disaster can create huge chaos to their productive systems.

Many economic researchers have focused on specific case studies to examine the macroeconomic consequences from various natural disasters. However, there are only very few studies that assess the impact of natural disasters on trade flows in 
the agricultural sector using a multi-country, multi-event framework. The only work to consider the effects of natural disasters (hurricanes) on the Caribbean agricultural exports is Mohan and Strobl (2012). Their study concluded that natural disasters cause severe disruptions to a country's agricultural exports, and the effects are amplified in small developing states where most depend on exports as their primary growth strategy.

This study seeks to follow their work but to a richer extent. First, it considers not only Caribbean small island nations but also Central American nations. On the one hand, most countries in Central America are similar to the Caribbean nations in terms of location, population, share of the agricultural sector, and cultural background. On the other hand, this set of countries includes a more diverse mix, for example, Mexico which has large geographical dimensions and is a comparatively richer country, together with Haiti which is a small, extremely poor country. Second, it considers the role of institutional quality in determining the trade flows after natural disaster shocks. Although an adverse impact of natural disasters on trade flows is not surprising to find, of great importance is a careful examination of those economic factors that can lead us to having smaller losses and higher abilities to cope with the disasters.

In addition, the possibility that geography is a determinant of a country's economic performance has been considered in the literature only recently. Countries can interact with each other through various channels including trade flows, capital flows, technological diffusions, and other political or social policies. Trade agreement among neighboring countries, such as the North American Free Trade Agreement (NAFTA) and the European Economic Union (EEU), is a strong evidence to support the existence of spatial dependence. In this sense, a disaster shock in any country can affect not only its own economic activities but also those of its geographically 
close countries, and consequently have an impact on those countries' economic outcomes. As a result, we should not treat each country as an independent or isolated unit, and an effective approach to address this spatial relationship is to use spatial econometric method (Ramírez \& Loboguerrero, 2002).

The main goal of this study is to quantitatively investigate the impacts of natural disasters on agricultural imports and exports of the countries in the Caribbean and Central American regions from 1996 to 2011. The countries considered fit the definition of small developing nations and most of them are island nations or have a considerable coastline (such as Belize and Panama). In addition, all of these countries, with the exception of Mexico, rely considerably on agriculture production and have a poorly diversified economy. The years are considered according to data availability.

More specifically, this study consists of four major innovations. First, it complements the literature by testing the hypothesis regarding the effects of a natural disaster on a country's imports and exports. On the one hand, it is widely suggested that after a disaster strikes, the country's total imports increase because of the need for commodities and reconstruction. On the other hand, a country's total exports should decline due to the extensive damages that a catastrophe causes to its essential infrastructures, such as ports, roads, and airports. The findings are also true for trade flows in the agriculture sector. Second, it employs the spatial econometric method to account for spatial effects which are considered in the literature only very recently. The objective is to demonstrate the spatial dependence on international trade flows, i.e., whether a country's agricultural trade is influenced by the agricultural trade of its neighboring countries. Third, it examines the role of institutional quality in the determination of agricultural trade flows in the aftermath of a disaster. The objective is to investigate whether countries with better institutions are 
better at coping with a natural disaster. Lastly, the above macroeconomic impacts of hurricanes using an alternative dataset are examined. Hurricanes are identified as the most frequent type of disaster in Central American and the Caribbean regions, so having a better understanding of this particular disaster is invaluable. Using a different dataset also allows us to check the robustness of the main results.

The structure of the study is as follows: Section 3.2 reviews the literature in the related field. Section 3.3 introduces the data used in the study. Section 3.4 presents the model specification and the regression results. Section 3.5 presents two robustness tests using storm data and hurricane data, and Section 3.6 provides concluding remarks.

\subsection{Literature Review}

\subsubsection{The Impact of Natural Disasters}

Natural disasters, such as hurricanes, earthquakes, tsunamis, and floods, have an observable adverse impact on macroeconomic activities. The direct impact of a natural disaster is the immediate reduction of the amount of human and physical capital. The destruction of these input factors is then followed by disruptions in production and output, which consequently affect the trade performance or the balance of trade (Ghosh \& Mukhopadhyay, 2014). Gassebner, Keck and Teh (2006) presented one of the most comprehensive and aggregate studies to examine the impact of major catastrophes on international trade flows for more than 170 countries from 1962 to 2004. They reported that disasters reduce trade in both importing and exporting countries. Their study also distinguished between the effect on exports and imports 
and concluded that disasters have a negative impact on exports, whereas they have a positive impact on imports.

Moreover, literature has suggested that developing countries face much larger shocks to their macroeconomic activities following a disaster than developed countries. Although the dollar value of direct economic damages from natural disasters is generally higher in developed countries, direct and indirect economic damages are a much larger fraction of GDP in developing countries (Raddatz, 2009). For example, Mohan and Strobl (2012) noted that the Kobe earthquake in 1995 destroyed about $2.5 \%$ of Japan's GDP while the 2010 Haitian earthquake wiped out $125 \%$ of Haiti's GDP. Small economies also seem to be more vulnerable than larger ones to other indirect consequences (Noy, 2009).

Studies have also shown that countries in Central America and the Caribbean are particularly vulnerable to disasters. Two possible explanations can be considered. First, these countries are geographically located in the most hurricane-prone area in the world, experiencing a higher frequency of natural disasters. Mohan and Strobl (2012) mentioned that Grenada has been struck repeatedly by natural disasters in 2002 - 2005, wiping out $90 \%$ of the island's nutmeg and mace export sectors, which contributed to $22.5 \%$ of its merchandise exports at that time. Second, most of these countries are poorly diversified: their primary sectors are agriculture and tourism, both of which are very sensitive to weather-related catastrophes. Therefore, they have fewer resources to cope with disasters by themselves. For example, in Antigua and Barbuda, Belize, Guyana, and Panama, food exports account for more than $50 \%$ of their total exports, while in Aruba, Nicaragua, and St. Vincent and the Grenadines, they account for more than 80\%. Furthermore, when a disaster strikes, the situation is aggravated because most of the workforce works in the agricultural sector, which tends to be poor (Mohan \& Stobl, 2012). 
Natural disasters often destroy almost the entire export sector of the affecting countries in this region. Auffret (2003) analyzed the impact of disasters on 16 Caribbean and Latin American countries over the period 1970 - 1999 and found substantial declines in investment and output. Rasmussen (2004) studied the small island nations in the Eastern Caribbean and found an immediate reduction in output after the disaster. He also concluded that the larger the share of trade in a country affected by a disaster, the larger impacts on trade. Mohan and Stobl (2012) estimated the impact of hurricanes for 24 Central American and Caribbean countries. They concluded that in general hurricanes reduce agriculture exports by 0.0068 tons in the year that they strike and 0.0049 tons in the year following the strike.

Although catastrophic events adversely affect production and exports, the imports of the affected countries tend to increase. Gassebner, Keck and Teh (2006) stated that any major reconstruction or rebuilding of damaged infrastructures in the recovery process will possibly increase imports, because the required materials, technology and skills may need to come from abroad. The effect is bound to be larger if external financial assistance is also provided. The Minister of Agriculture of Belize reported that the impacts of a tropical storm in 1995, Hurricane Keith in 2000, and Hurricane Iris in 2001, resulted in reductions in agriculture production and exports but short-term increases in food imports. ${ }^{1}$ The reduced production of shrimp, lobster, papayas, and bananas produced a short-term fall in exports, and damages to fisheries and infrastructures produced a longer-term fall in production which led to a long-term decrease in exports. However, the shortages of domestic staple commodities, such as rice, corn, and beans, contributed to an increase in imports.

\footnotetext{
${ }^{1}$ From speech of "His Excellency Daniel Silva, Minister for Agriculture, Fisheries and Cooperatives of Belize" at the World Food Summit: Five Years Later (June 10-13, 2002).
} 


\subsubsection{The Role of Institution}

Natural disasters are known for their sudden destructiveness and can thus serve researchers and policymakers as natural experiments to conduct analysis without further needs for other assumptions. Baker and Bloom (2013) referred this as disaster shock methodology. Previous literature has identified many different socioeconomic factors that can help economies better cope with disaster strikes. Kahn (2005) showed that countries with higher per capita income experience on average less death tolls from disastrous events. Lower income inequality also leads to lower fatality rates. Toya and Skidmore (2007) found that countries with higher educational level, higher income level, more complete financial systems, and smaller government experience fewer losses. Gassebner, Keck, and Teh (2006) stated that the smaller and less democratic a country, the more are its trade flows reduced in case it is struck by a disaster.

Only a few years ago, the role of institutional quality on disaster-related economic losses was finally considered in the literature. North (1990) suggested that countries with better institutions and less distortionary policies invest more in human and physical capital and apply their existing factor endowment more efficiently. This could also apply to a country's effort to mitigate the effects of natural disasters. Kahn (2005) considered the interaction between institutional quality and the death tolls from earthquakes and found that countries with higher quality institutions suffer less death from natural disasters. Raddatz (2007) studied the interaction with output volatility caused by natural hazards. He stated that countries with good institutions are better at dealing with the distributional consequences of exogenous shocks and also tend to follow more sound macroeconomic policies when facing exogenous contingencies. 
Rashky (2008) considered institutions in a much broader sense, including not only governments but also NGOs, charities, and the financial systems among others. He highlighted the importance of good quality institutions as an additional socioeconomic factor to provide protection against natural disasters. Moreover, he argued that the quality of institutions determines the key aspects of post-disaster relief. Overall, the literature concurs that countries with better institutions suffer from less victims and lower economic damages from disaster shocks.

Studies on the relationship between institutional quality and international trade are relatively easier to identify. Levchenko (2004) showed that institutional differences are important determinants of trade flows. Borrmann, Busse, and Neuhaus (2006) demonstrated that countries with high quality institutions are more likely to benefit from trade. Nunn (2007) found that countries with good contract enforcement specialize in industries where relationship-specific investments are most important. He concluded that the ability to enforce contracts is an important determinant as a source of comparative advantage.

Moreover, a strong positive link between quality of institutions and exports is observed. Using a panel of countries between 1920 - 2000, Méon and Sekkat (2006) observed that exports of manufactured goods are positively affected by the higher quality of institutions. A study by Francois and Manchin (2006) supported the notion that a country's export performance and the propensity to take part in the trading system depend on its institutional quality. However, the relationship between institutional quality and imports is not clearly observed in the literature.

To sum up, in small developing countries (especially the Caribbean), natural disasters - mostly hurricanes or tropical storms - lead to a short-term increase in imports and both a short-term and a long-term decreases in exports. The increase in imports is due to the shortage of staple commodities such as rice and corn. The 
negative effect on exports is due to the damage to the country's essential infrastructures, such as airports, ports, and roads. Moreover, countries with higher quality institutions are more likely to benefit from trade. At the same time, these countries experience less human deaths and lower economics losses from natural disasters.

\subsection{Data}

\subsubsection{Agricultural Trade Data}

The data on agricultural exports and imports are taken from the Food and Agriculture Organization of the United Nations (UNFAO), which is the largest and most comprehensive food and agricultural database globally. It records large crosssectional and time-series data relating to hunger, food, and agriculture for 245 countries and 35 regional areas from 1961 to 2011. As to the agricultural trade data, UNFAO gives detailed information for export/import value base quantity or base

price over 575 agricultural products. Exports and imports value base quantity data for total products are used in this study. As a result, a sample of 21 countries 7 Central American countries and 14 Caribbean countries - is constructed for the period 1996 - 2011. Some years are missing for a particular country due to data availability, and the list of countries is shown in Table 3.1.

\subsubsection{Natural Disasters Data}

- Data Source and Definition

The data on natural disasters used in this study are taken from the Center for the Research on the Epidemiology of Disaster (CRED). Its emergency event database (EM-DAT) records essential core data on the occurrences and consequences of more 
than 18,000 disasters in the world from 1900 to the most recent. It is the most accessible and largely used database for studies on the impact of disasters.

According to CRED, a disaster can be defined as a natural situation or event which overwhelms local capacity, necessitating a request for external assistance. For a disaster to be recorded into the EM-DAT, at least one of the following criteria much be satisfied: (1) ten or more people reported killed; (2) hundred or more people reported affected; (3) declaration of a state of emergency; (4) call for international assistance. The database also distinguishes between two main categories of disasters: natural disasters and technological disasters. The natural disaster category is further divided into 5 subgroups, which in turn cover 12 types and more than 30 subtypes, as summarized in Figure 3.1. The technological disaster category includes industrial accidents (such as chemical spills and gas leaks) and transportation accidents (such as plane crashes and road accidents). However, the impact of technological disasters is beyond the scope of this study and will not be examined. ${ }^{2}$

In our sample of 21 Central American and Caribbean countries, there are 554 reported natural disasters over the study period (see Table 3.1 column (1)). Figure 3.2 Map 1 presents the location and the number of disasters occurred for each country. Although most of these countries share similar geographic locations and reside relatively close to each other, some countries still experience disastrous events more frequently than others. Furthermore, among all the disaster types, 226 tropical storms are recorded, which consist of $41 \%$ of the total events, making it the most frequent type of disaster in this region. As we know, a storm system carrying strong winds and heavy rain can cause serious damages, notably to automobile, aircraft, international shipping, livestock, and most commonly, farmers' crops. A storm can

\footnotetext{
${ }^{2}$ The study by Gassebner, Keck and Teh (2010) found that whether or not the technological disasters are excluded does not alter the estimation results.
} 
also cause significant inland flooding as well as producing extensive coastal flooding up to 25 miles from the coastline. ${ }^{3}$ Against this background, a more detailed study on the impact of storms on a country's macroeconomic activities is necessary.

- The Decision Rule

Following the study by Munich Re (2006), a decision rule should be adopted to filter the disasters in order to quantitatively examine the impact of a disaster to its affected countries. The magnitude of a disaster matters for the reason that there should be a certain size to affect economic activities. Although small-sized disasters are observed to have certain impacts on life and property, they might not be empirically significant on the aggregate level. However, disasters with large magnitude can directly cause substantial amount of casualties and damages to production facilities and infrastructure (export side). Consequently, the damage and loss could induce significant recovery expenditures and a large inflow of international assistance (import side).

The EM-DAT database does not provide information that can directly depict the size of a disaster, such as the wind speed for a storm or the Richter scale for an earthquake. However, it reports four measures that may help determine the severity of a disaster: the number of people killed, the number of people injured, the number of people affected, and an estimated economic damage (in thousands of US dollars). Based on these measures, Munich Re (2006) classified disasters into different categories: a small-scale loss involving fewer than 10 deaths and no damages, a devastating catastrophe involving more than 500 deaths and damage in excess of $\$ 500$ million, and finally a great natural catastrophe involving thousands of deaths and extreme insured losses.

\footnotetext{
${ }^{3}$ See "Tropical Cyclone" in Wikipedia, retrieved Nov 12th, 2013, available online: http://en.wikipedia.org/wiki/Tropical_cyclone.
} 
In this study, we are more interested in understanding the impact of large-scale disasters on agricultural trade flows, from the aggregated perspective. Due to the fact that countries in Central America and the Caribbean are relatively small lowincome countries, the extreme high criterion of a large population loss or monetary loss does not apply. Therefore, a disaster is considered as large-scale in this study if it involves more than 20 deaths, more than 10,000 people affected, and damage in excess of $\$ 50$ million. The category meeting this criterion is defined as a severe catastrophe in Munich Re (2006). The total number of severe catastrophes has decreased to 511 in our sample after adopting the decision rule, and the number of occurrences for each country is listed in Table 3.1 column (2). As shown in column (3) of the same table, the total number of large-scale storms is reduced to 191, and the number of occurrences for each country is also listed. Note that the storm data are a subset of the total disaster data in this study.

\section{- Alternative Hurricane Data}

An alternative database from the National Hurricane Center (NHC) is used to study the impact of major hurricanes on agricultural trade flows in Section 3.5. NHC is a component of the National Centers for Environmental Prediction (NCEP) located at Florida International University (FIU) in Miami, Florida. Its tropical cyclone reports contain comprehensive information on each named hurricane, including its synoptic history, meteorological statistics, and the post-analysis best track. More importantly, NHC's best track dataset provides six-hourly reports on hurricane positions and intensities, which offer a more accurate information to count and locate all the affected countries during a particular hurricane strike. This is a major advantage compared to the EM-DAT database, which only records a storm based on human losses and direct economic damages. Figure 3.2 Map 2 provides the 
tracks of major hurricanes in this region during 1996 - 2011, and it is clearly shown that almost all of the studied countries have suffered from the impact of hurricanes. Some countries may experience a direct strike of a strong storm, but others may be affected to a limited extent as the hurricanes remained just offshore.

\subsubsection{Institutional Quality Data}

The data on institutional quality are taken from Worldwide Governance Indicators project (WGI) by World Bank (WB). The database records aggregate and individual governance for 215 countries over the period 1996 - 2012 for six broad dimensions of governance: voice and accountability, political stability, government effectiveness, regulatory quality, rule of law, and control of corruption. The first two indicators tend to capture the process by which governments are selected, monitored, and replaced; the third and fourth indicators tend to capture the capacity of the government to effectively formulate and implement sound policies; and the last two indicators tend to capture the respect of citizens and the state for the institutions that govern economic and social interactions among them. ${ }^{4}$

Until the year of 2002, the indexes were calculated and reported only every other year. Therefore, the values in 1997, 1999, and 2001 in this study are generated and used by taking the average values in the previous and the following year. The composite measures of governance indicator created are in units of a standard normal distribution, with a mean of zero and a standard deviation of one. As a result, the indexes range from approximately -2.5 to 2.5 , with higher values corresponding to better quality institutions. WGI constructed this governance scale based on over 30 different data sources that report the views and scores on the quality of various

\footnotetext{
${ }^{4}$ For more detailed definition of each indicator, see http://info.worldbank.org/ governance/wgi/index.aspx\#doc.
} 
aspects of governance. Therefore, the indexes are expected to provide the most reliable and precise data for the quality of institutions.

\subsection{Empirical Analysis}

\subsubsection{Model Specification}

The main goal of this study is to estimate the impact of large-scale natural disasters on agricultural trade flows in the Central American and Caribbean regions from 1996 to 2011. Accordingly, a panel econometric model is used as the baseline regression, expressed as:

$$
\begin{gathered}
\operatorname{Ln}\left(A G R_{i t}\right)=\sum_{m=0}^{3}\left[\alpha_{m} D_{i t-m}\right]+\beta X_{i t}+v_{t}+\varepsilon_{i t} \\
X_{i t}=X_{i t}\left(G D P_{i}, I N V_{i}, F D I_{i}, G O V_{i}, \text { POP }_{i}, \text { LAND }_{i}, \text { OPENness }_{i}\right)
\end{gathered}
$$

The dependent variable $\operatorname{Ln}\left(A G R_{i t}\right)$ is the natural logarithm of agricultural trade flows (including imports $L n A g^{i}$ and exports $L n A g^{e}$ ) for country $i$ at time $t$. $D_{i t-m}$ denotes the disaster dummy that takes the value of one if a disaster affects county $i$ on year $t-m$ and zero otherwise. The disaster effect is considered over a three-year window, beginning in the year immediately after the event. ${ }^{5}$ More precisely, $\alpha_{3}$, the coefficient of $D_{i t-3}$, will capture the impact of a disaster on trade flows if it occurred three years ago. As suggested by the literature, the expected sign of $\alpha$ should be positive for agricultural imports and negative for agricultural exports.

In this study, we are also interested in examining the spatial effects of a country's agricultural trade flows. The spatial dependence among countries is therefore included in order to find out if the trade flows in one location depend on the trade

\footnotetext{
${ }^{5}$ Some of the case studies in the literature have used a similar horizon. They considered the short-run effect as within three years (Benson \& Clay, 2004) and the long-run effect for more than five years (Mechler, 2009).
} 
flows at other geographically close locations. As a result, a spatial panel econometric model is estimated:

$$
\operatorname{Ln}\left(A G R_{i t}\right)=\rho\left(W * A G R_{i t}\right)+\sum_{m=0}^{3}\left[\alpha_{m} D_{i t-m}\right]+\beta X_{i t}+v_{t}+\varepsilon_{i t}
$$

where $\rho\left(W * A G R_{i t}\right)$ is the spatial lag of the dependent variable added to the model in order to account for spatial dependence. $W$ is the spatial weight matrix that measures the spatial relationship among partner countries. $\rho$ is the estimated coefficient to test spatial correlation. A positive/negative sign of $\rho$ indicates that a country's trade flows (the dependent variable) are positively/negatively influenced by trade flows of its partner countries.

It is also useful to discuss the ways to construct the spatial weight matrix $W$, which specifies the partner countries considered in the estimation. In this case, two setups are employed:

$$
\text { (1) } W_{i j}^{C} \begin{cases}1 & \text { if countries } i \text { and } j \text { share a border } \\ 0 & \text { otherwise }\end{cases}
$$

(2) $W_{i j}^{N N K} \begin{cases}1 & \text { if countries } i \text { and } j \text { are } K \text { nearest neighbors } \\ 0 & \text { otherwise }\end{cases}$

where $K=1,2$, or 4 .

The first setup is a simple contiguity matrix (see equation (3.4)). Each element in matrix $W, W_{i j}^{C}$, takes the value of one if countries $i$ and $j$ share a common boundary and zero otherwise. In other words, countries which share the same border are partner countries in this setup. However, because a majority of countries in the sample are small island countries, using a common border may not represent the spatial correlation in a more realistic way. Therefore, in the second setup, partner countries are redefined as nearest neighbors. As specified in equation (3.5), each 
element, $W_{i j}^{N N K}$, takes the value of one if countries $i$ and $j$ are nearest neighbors $(K=1)$, whether they share a common border or not. The second setup also introduces information regarding the neighbors' neighbors, that is, countries are considered as partner countries if they are second nearest neighbors $(K=2)$ or fourth nearest neighbors $(K=4)$. Such a setup is necessary when we assume the process of spatial effects over time. It is possible that the initial effects in a country can impact not only its neighboring countries, but over time also its neighbors' neighbors.

Finally, in order to examine the role of intuitional quality in determining a country's ability to cope with disasters, the institutional variable is added to both the non-spatial panel model and the spatial panel model.

$$
\begin{gathered}
\operatorname{Ln}\left(A G R_{i t}\right)=\sum_{m=0}^{3}\left[\alpha_{m} D_{i t-m}+\lambda_{m} Q_{i t}+\gamma_{m} D_{i t-m} * Q_{i t}\right]+\beta X_{i t}+v_{t}+\varepsilon_{i t} \\
L n\left(A G R_{i t}\right)=\rho\left(W * A G R_{i t}\right)+\sum_{m=0}^{3}\left[\alpha_{m} D_{i t-m}+\lambda_{m} Q_{i t}+\gamma_{m} D_{i t-m} * Q_{i t}\right]+\beta X_{i t}+v_{t}+\varepsilon_{i t}
\end{gathered}
$$

The parameter $Q_{i t}$ represents the institutional quality, and its interaction term with the disaster dummy variable $\left(D_{i t-m} * Q_{i t}\right)$ is our coefficient of interest. The coefficient $\gamma_{m}$ tests whether the impact of a disaster on imports or exports depends on the quality of institutions. Since the institutional quality may also affect trade flows directly, the specification includes $Q_{i t}$ as well. According to the literature, a country with better institutions is better at coping with disasters. We expect the sign of $\gamma_{m}$ to be negative, as the higher the institutional quality, the less impact of a disaster on trade flows.

The variable $X_{i t}$ is a set of control variables that affects trade flows, including income per capita, the ratio of investment to GDP, foreign direct investment, government consumption, trade openness, population size, and land size. The data for 
these variables are mainly taken from the World Bank database. The variable $V_{t}$ denotes the year effect, and $\varepsilon_{i t}$ is a residual term. The definitions and sources of all variables used in this study are summarized in Table 3.2.

\subsubsection{Choice of Institution Indicators}

As shown in Table 3.3, all of the six indicators of institutional quality from WGI are highly correlated, with the highest correlation coefficient of 0.9 and the lowest over 0.6. Méon and Sekkat (2006) observed that exports of manufactured goods are positively affected by the control of corruption, the rule of law, government effectiveness, and the lack of political violence. Their instrumental variable regressions confirmed that the control of corruption, instead of other dimensions of governance, robustly affects manufactured exports. Based on their finding, this study first tests whether the indicator of control of corruption can represent the quality of governance better among others after a disaster.

In order to do so, multiple non-spatial panel regressions are estimated with agriculture export as the dependent variable and together with each of the six indicators as the institutional explanatory variable. ${ }^{6}$ As shown in Table 3.4 column (4), the interaction terms of control of corruption with disaster dummy appear to be the most significant and of the largest magnitude over the three-year horizon. This demonstrates that, compared with other available measures, a more efficient institution at controlling for corruption results in higher resilience in agriculture exports when a disaster strikes the country.

\footnotetext{
${ }^{6}$ The coefficients for all six governance indicators are insignificant when using agriculture import as the dependent variable, and the results are not reported for brevity. Further discussions will be provided in the next section.
} 
Therefore, control of corruption is selected as the main institutional quality variable for the rest of the regression analysis. According to WGI, this indicator measures the extent to which public power is exercised for private gain, including both petty and grand forms of corruption, as well as state power by elites and private interests. It also measures the strength and effectiveness of a country's policy and institutional framework to prevent and combat corruption.

\subsubsection{Regression Results}

In this section, the estimation results for a disaster shock using a panel of 21 countries observed from 1996 - 2011 are presented. Table 3.5 reports the estimation results on the impact of disasters on agricultural imports and Table 3.6 on agricultural exports. Table 3.7 presents the estimation results for both agriculture imports and exports with the inclusion of the institutional variable.

- The Impact on Agricultural Imports

The estimation results for the non-spatial panel econometric models are presented in the first two columns of Table 3.5. According to Column (1), there is a significant positive effect of a large-scale disaster on agriculture imports. Accordingly, imports are increased by $5.9 \%$ in the same year when a disaster strikes the country, and this is significant at the $5 \%$ level. Column (2) continues to examine the positive impact when the calamity took place in the previous years. As shown, the positive effect is even higher: a $6.4 \%$ increase in imports in the one lagged year, at the $5 \%$ significance level.

These results are in line with the literature that finds that disasters can induce more imports since countries facing disasters will have an increased demand for foreign assistance and have increased recovery expenditures. The vast majority 
of the countries in our sample, with Mexico being the notable exception, are small developing countries which under normal circumstances barely produce enough food locally (in particular, corn, maize, drinking quality water, and dairy products) for their daily consumption. As soon as a natural disaster occurs, they must resort to necessary staple products, which will further increase the agricultural imports after the disaster. In the following year, higher need for reconstruction including the agricultural industry will stimulate higher imports from foreign countries. The huge share of the agricultural sector in the countries' GDPs is evidence that most of the reconstruction money will be spent either in rebuilding essential infrastructure or in renovating the capital stocks that were destroyed. Moreover, note that for a disaster that occurred two years ago, there are no further impacts on agriculture imports. This implies a possibility that the reconstruction of the agricultural sector usually takes one year or less than two years after a catastrophe.

The estimation results for the spatial panel econometric models are also presented in Table 3.5. Column (3) presents the results given the spatial weight matrix of contiguity. As shown, the coefficients of the disaster variables are significant for all three years after disaster shocks but not the same year of the shock, which is not consistent with the results using non-spatial models. However, when using the spatial weight matrix of nearest neighbors (see column (4)), the coefficients of disaster variables become consistent with the non-spatial models and our theoretical expectation. This suggests that the second setup of the spatial weight matrix using nearest neighbors is a more appropriate specification than the first simple setup using a common border, given the unique geographic features of the countries in our sample. Moreover, columns (5) and (6) present the estimation results given the spatial weight matrix of second nearest neighbors and fourth nearest neighbors, respectively. It is clearly shown that natural disasters have a significant impact on 
agricultural imports through time when controlled for the spatial effects, indicated by the significant coefficient for a disaster that occurred two years ago. Finally, the coefficients of the spatial lag variable $(\rho)$ are highly significant in all spatial models, confirming the existence of spatial dependence in agricultural imports. The positive sign suggests that a country's imports in the agricultural sector are positively correlated to the imports of its partner countries.

- The Impact on Agricultural Exports

Table 3.6 reports the estimated coefficients of the disaster variables with agriculture export as the dependent variable, using non-spatial panel models (see columns (1)-(2)) and spatial panel models (see columns (3)-(6)). It is interesting to notice that we cannot reject the null hypothesis that $\alpha_{0}=0$ under standard significant levels. This indicates that large-scale disasters do not affect agriculture exports in the year they take place. The reason is possibly related to the timing of the disasters in the Caribbean and Central American regions. If most of the natural disasters occur at the end of the accounting period, for example, the hurricane season in the Atlantic Ocean begins in June 1st and ends November 30th, most of the countries' products were already exported in the first three quarters of the year. If so, the result is in agreement with the fact that disasters do not appear to affect agricultural exports in the current year. Nonetheless, their impacts are accounted for in the next year's outcomes which show on average a significant decline in the total amount of agricultural exports (in the year following a natural disaster).

Further experiments with the inclusion for two and three lagged years are also shown. Accordingly, natural disasters are found to have significant year-lagged negative effects. The country experiencing a large-scale disaster shock results in a reduction of $15.2 \%$ in agriculture exports one year after. The reduction is even more serious two years after the shock: a $17.5 \%$ decrease in the level of exports. In the 
third year, the impact is slightly lower, a $17.2 \%$ decrease, but still obvious. These findings are similar to those of another study that investigated the hurricane impact on sugar exports in the Caribbean from 1700 - 1960 (Mohan \& Strobl, 2012). They found no significant effect on sugar exports in the year when a hurricane strikes, but a significant reduction by $25 \%$ on average in the one lagged year. Finally, the significant signs of the spatial lag variable in all spatial models confirm the existence of spatial dependence. The agricultural exports among partner countries are positively correlated.

\section{- The Role of Institution}

Table 3.7 presents the regression results with the inclusion of the institutional indicator: control of corruption. The inclusion of the interaction term does not alter the estimation results to any major extent. For example, the coefficients of spatial lag variable are still positively significant for both agricultural imports and exports. Columns (1)-(3) present the effects of institutional quality on agricultural imports. Notice that there is a significantly positive coefficient of control of corruption that increases agricultural imports by $11.5 \%$. However, all the interaction terms appear to be insignificant. For any year followed by a disaster, the level of institutional quality does not matter in altering the impact of a disaster on countries' imports.

There are some possible explanations for such a finding. First, the measure for corruption that we use here only captures the control for corruption within the country, from the perspective of institutional performance. It does not, however, reflect the corruption activity levels on the border. Plenty of literature has studied the effects of corruption on reducing international trade (de Jong \& Udo, 2006; de Jong \& Bogmans, 2011) and considered corruption on the border using different measures (such as waiting time at the border, or quality of the customs office). In 
addition, we need to take into consideration the peculiar geographical characteristics of our sample. Most of the countries are small ones with extremely permeable borders and it is highly possible that there are no strict customs regime at the border. Furthermore, the control of corruption variable may not capture the type of trade we studied because it remains unaccounted for in the national statistics. It is also possible that due to the increase in the level of imports, there are not enough government officials to control them. Therefore, the geographic characteristic of our sample suggests that corruption at the institutional level may not reflect the corruption at the border, and also there may not be corruption at the border at all since the borders' controls are easily evaded.

Moreover, it could be possible that the level of institution does not affect the impact of disasters on imports, because the affected countries must import necessary products for survival regardless of the quality of institutions. The measures for quality of institutions are used to examine normal circumstances, but not for a natural disaster situation. For instance, "Rule of Law", a key indicator for institutional quality, is not going to significantly affect the total volume of agricultural products most of which are necessary for survival after the catastrophe. In less technical terms, suppose the whole corn producing industry was wiped out for a particular country due to a natural disaster, it must import this staple products for humanitarian reasons no matter if it has a relatively good or bad quality of institution. Under this assumption, it is logical that the institutional quality does not determine the impact of natural disasters on the level of agricultural imports.

The estimation results on the effects of institutional quality on agricultural exports are presented in columns (4)-(6). The inclusion again does not alter much the effect for agricultural exports compared to the baseline regression. The linear relation between control of corruption and exports is still positively significant. There 
remains no impact on exports in the current year, neither by a disaster strike (the disaster dummy), nor by the quality of institution (the interaction term). The effect is revealed from one lagged year indicated by the significantly negative coefficient. After one or more years, agricultural exports are adversely affected by the disaster. However, the effect is less pronounced due to better quality institutions. The higher institutional quality plays the most significant role in the second year after the strike. For each additional disaster, the negative slope on exports is decreased by $14.5-16.9 \%$. The institutions with better control for corruption help the affected countries cope with disasters in an effective way. Nevertheless, even in countries with the best quality indexes, the disaster reduces exports.

Our models also control for country-specific characteristics. We find that the level of GDP is highly significant at the $1 \%$ significance level. In fact, a $1 \%$ increase in GDP translates to an approximate $50 \%$ increase in the total amount of agricultural imports and exports. Similarly, countries with higher levels of investment, higher levels of FDI, and lower levels of government spending are associated with a higher level of trade flows. Total population, land size, and openness are found to be less significant, but they are useful to improve the explanatory power of the model. Dropping any one of these variables decreases the model of fit $\left(R^{2}\right)$ sharply.

In summary, severe disasters indeed have an impact on agricultural trade flows. The agriculture import variable reacts immediately following the disaster with an increase of $6 \%$ on average. In addition, both disaster shocks and quality of institutions matter in determining the effects of disasters on agriculture exports. The impact is revealed not immediately but after one year and followed with a reduction ranging from 11-19\% - being larger for lower quality institutions. Finally, a country's trade flows are positively influenced by the trade flows of its partner countries. 


\subsection{The Impact of Storms and Hurricanes}

Tropical storms and hurricanes are endemic to the Central American and Caribbean regions. They are the most damaging and most frequent natural disasters that affect the region. A close study focusing on the impact of storms and hurricanes on agricultural imports and exports is provided in this section.

Moreover, it should be noted that so far we only used the disaster shock as a dummy variable in our analysis, but there are other ways to empirically examine the disaster impacts. One alternative approach is to use the count variable, which $D_{i t}$ now denotes the frequency of disasters satisfying the decision rule for each country $i$ on year $t$. This approach allows us to obtain a more precise estimation of the disaster impact. Countries, which are struck more than once by a severe disaster in the same year, are expected to suffer higher economic losses. This frequency disaster effect using a count variable thus captures additional information by distinguishing countries which suffer one single incident and multiple incidents. Therefore, in this section both the dummy effects and frequency effects for storms and hurricanes are employed and compared.

\subsubsection{The Impact of Storms}

In order to examine the impact of storms, we use the publicly available storm data from the EM-DAT database. Since the storm data are also a subset of the natural disaster data, they can serve as a robustness check for the baseline regression. The impact of hurricanes using an alternative dataset as the second robustness check will be discussed later.

Table 3.8 shows the impact of severe storms on agricultural imports and exports, and the results confirm our findings in the main specification. For the dummy effects 
(see columns (1)-(4)), imports significantly increase by about $7.6 \%$ if the country experienced a severe storm in the same year and by about $8.2 \%$ in the year after. However, the effect becomes insignificant in the second year. Also, the level of institutional quality does not play an important role in determining the outcome of imports. The expected positive sign in imports after a storm is in line with our main results using all types of natural disasters. Since a catastrophic storm wipes out a country's essential infrastructure, that country can only resort to import commodities which it needs for survival. This will inevitably lead to an increase in imports. One years after, the affected country's reconstruction is under way which is likely to further induce a rise in the total quantity of imports the country needs.

The effects on exports remain significant and negative, starting from the one lagged year. However, the magnitude of the impact is lower compared with that of all severe natural disasters. This is because other types of catastrophic events, such as earthquakes and floods, which seriously reduce agricultural exports are excluded in this analysis. However, the magnitude of the interaction terms between storms and institutional quality appears to be higher. This demonstrates that, in contrast with other types of disasters, a high quality institution is especially influential in supporting the country's export performance. On average, during the three years after a storm strikes, exports decrease by $8.1 \%, 14.23 \%$ and $10.07 \% .^{7}$

Lastly, the results for the frequency effects further confirm the robustness of the baseline regression. However, it is worth pointing out that all the coefficients of storms on agriculture imports become significant. The impact of an additional storm not only increases agricultural imports in the year after (the most by $4.1 \%$ ), but also increases agriculture imports in the second and the third year (by $2.6 \%$ and

\footnotetext{
${ }^{7}$ This result is similar with another study by Croward (2000), where he found that the most severe storms and hurricanes experienced in the Caribbean between 1974 and 1996 caused a reduction of $10 \%$ in merchandise exports.
} 
$3.6 \%$, respectively). This finding depicts the countries which suffer repeated storm strikes and thus constantly demand recovery and foreign assistance. In the end, all the signs and the magnitude of the coefficients on exports are in accordance with our previous findings.

\subsubsection{The Impact of Hurricanes}

The islands of Caribbean have been singled out as the most disaster-prone territory in the world on account of the huge number of hurricane strikes experienced (Mohan \& Stobl, 2012). The names of several devastating hurricanes have been recorded in history as the deadliest hurricanes in the world. For example, Hurricane Mitch, the most powerful and most destructive hurricane of the 1998 Atlantic hurricane season, struck Honduras and other Central American countries and caused them $50 \%$ of total damage in the agricultural sector. Honduras requested nearly four billion dollars to pay for its Master Plan for National Reconstruction and Transformation (Duran, 1999). Moreover, hurricanes are ranked as one of the most deadly and costly natural disasters in the world. Thus, quantitatively estimating the impact of named hurricanes on small developing countries is of great importance.

The hurricane data in our study are taken from NHC database. According to NHC, government weather services will assign a name to a tropical storm system that reaches certain intensity (maximum sustained winds between 34 knots/39 mph and 64 knots $/ 74 \mathrm{mph}$ ). Table 3.1 column (4) provides the number of hurricanes for each country. Note that the numbers do not necessarily match the storm data from EM-DAT, because NHC defines an impacted area or country depending on the six-hourly tracked hurricane path. Whether a hurricane causes economic damages to a country directly or indirectly is not a major consideration. As we know, some 
hurricanes directly strike one country but others only pass through without causing substantial economic losses. The most illustrating examples are the Bahamas and Cuba. It is demonstrated that there are more hurricanes recorded in NHC database than the storms recorded in EM-DAT. This is because many hurricanes in the Eastern Caribbean are formed in the Bahamas or Cuba, but they only strengthen and intensify after leaving the area and moving north. For instance, the infamous Hurricane Katrina formed over the Bahamas on 2005 and crossed southern Florida as a moderate Category 1 hurricane, causing some deaths and flooding there before strengthening rapidly in the Gulf of Mexico. Katrina then strengthened and turned into a Category 5 hurricane over the warm Gulf waters and caused severe destruction along the Gulf coast from Central Florida to Texas. ${ }^{8}$ As a result, the Bahamas is listed as an affected country due to Hurricane Katrina in the NHC database but not the EM-DAT database.

To further verify our previous findings as a second robustness check, the hurricane data are used and the results are provided in Table 3.9. First, a large long-lasting impact of hurricanes is observed on agriculture imports. A $6.7 \%$ increase in imports is evidenced immediately when a hurricane strikes the country. This finding is similar to the earlier results. However, the impact on imports seems to last longer and with a higher magnitude: a rise by $8.16 \%$ if there was a hurricane three years ago. This result is statistically significant at the $1 \%$ level. Similar results are found when we use the count variable (frequency effects). Rappaport (2009) from NHC stated that the next time you hear hurricane, think inland flooding. Inland flooding has been responsible for more than half the deaths associated with hurricanes. Compared to other types of disasters, it especially destroys buildings and other structures, such

\footnotetext{
${ }^{8}$ See "Hurricane Katrina" in Wikipedia, retrieved Nov 17th, 2013, from http:// en.wikipedia.org/wiki/Hurricane $\backslash$ Katrina.
} 
as bridges, sewerage systems, roadways, and canals. These damages often take a longer period to be repaired, which might be one interpretation given our results.

The results on agricultural exports also confirm the robustness of our previous findings. Hurricanes destroy exports in the agricultural sector, especially from the second year to the third year. With a lower quality institution, the adverse impacts are more pronounced. Note that the interaction term with the hurricane dummy and exports appears to be significant in the current year, at the $10 \%$ level. The role of the institution is revealed as soon as the hurricane strikes the country. The coefficients of the interaction terms remain highly significant for the frequency effects. Facing an additional hurricane shock, the institution with better quality mechanisms matters for a country when considering economic activities.

\subsection{Conclusion}

This study aims to present a detailed analysis on the impact of natural disasters on agricultural trade flows (agricultural imports and exports) for the Caribbean and Central American regions from 1996 to 2011. We find that in the aftermath of a natural disaster, agricultural imports increase by approximately 6\%. Agricultural exports, on the other hand, are reduced by 11-19\% starting from the year after. Furthermore, we also find that in general, having better quality institutions enables the country to overcome the adverse effects of a catastrophe sooner. In fact, countries with better institutions also experience lower decreases in agricultural exports. A close study on the effects of storms and hurricanes using different datasets is in line with this conclusion.

This study is expected to contribute meaningfully to the literature on the macroeconomic implications of natural catastrophes. First, it sheds light on the fact that 
agricultural imports increase and exports decrease following a disaster shock. Hence, small developing countries need to focus at either reducing their export vulnerability to disasters in the first place, or at mitigating the adverse export impact of disasters when they occur. Second, it extends the studies in the literature by including a broader and more diverse set of countries and considering the importance of having good quality institutions. The results indicate that enhancing government effectiveness, reaching political stability, and controlling for corruption can be beneficial. We do not have many choices when facing a devastating catastrophe, but having effective and good quality institutions will prevent us from having higher human and economic losses in the aftermath of a disaster. Finally, the results indicate that a country's trade flows are indeed affected by the performance of its neighbors. The spatial dependence across countries should not be ignored in the analysis of economic activities at the country level. Disaster risks affect mainly the poor, so economic growth is the key to reducing these risks. Thus, cooperation agreements among neighboring countries are essential for the economic growth of the regions.

Even though the data for disasters are publicly available and contain massive information, more precise data are still needed to study the disaster impacts in greater detail. In particular, less aggregated data, such as weekly, monthly or quarterly disaster data, might be useful. Different types of disasters, other than storms or hurricanes, should be addressed and examined in future studies. For example, meteorological disasters (such as storms) are more frequent and predictable, so it is possible for people to evacuate the affected region, and preventative measures can be taken beforehand. Geophysical disasters (such as earthquakes) are less frequent and hard to predict in their occurrences. Therefore, meteorological and geophysical disasters may influence factors of production very differently and hence have different effects on agricultural trade flows. 
Finally, the natural disaster-related studies could be augmented by examining the impact on a broader definition of development, such as investment, consumption, and growth rate, in addition to agricultural trade flows. As the data become more available for small and poor countries, studying the disaster effects will be possible, potentially lending support for disaster assistance programs or institutions and eventually better helping people survive the disasters. 


\section{References}

Auffret, P. (2003). High consumption volatility: The impact of natural disasters. World Bank Policy Research Working Paper WPS/2962, Washington, D.C.

Baker, S., \& Bloom, N. (2013). Does uncertainty reduce growth? Using disasters as natural experiments. NBER Working Paper No. w19475

Benson, C., \& Clay, E. J. (2004). Understanding the economic and financial impacts of natural disasters. Disaster Risk Management Series No. 4, World Bank, Washington DC.

Borrmann, A., Busse, M., \& Neuhaus, S. (2006). Institutional quality and the gains from trade. HWWA Discussion Paper No. 341. Available Online: http://dx.doi.org/10.2139/ssrn.895242

Croward, T. (2000). Comparative vulnerability to natural disasters in the Caribbean. Caribbean Development Bank, Staff Working Ppaer No.100. Available Online at: http://www.caribank.org/uploads/publications-reports/staff-papers/ wkgppr_1_natural_disasters $\backslash \% 5 \mathrm{~B} 1 \backslash \% 5 \mathrm{D}$.pdf

De Jong, E., \& Bogmans, C. (2011). Does corruption discourage international trade? European Journal of Political Economy, 27(2), 385-398.

De Jong, E., \& E. Udo. (2006). Is corruption detrimental to international trade? Working paper, Radbopund University, Nijmengan.

Duran, J. R. (1999). Development- Honduras: Hurricane Mitch - One year after. Inter Press Service News Agency. Available Online: http://www.ipsnews.net/ 1999/10/development-honduras-hurricane-mitch-one-year-after/

Francois, J., \& Manchin, M. (2013). Institutions, infrastructure, and trade. World Development, $46(\mathrm{C}), 165-175$.

Gassebner, M., Keck, A., \& Teh, R. (2010). Shaken, not stirred: The impact of disasters on international trade. Review of International Economics, 18(2), 351-368.

Ghosh, T., \& Mukhopadhyay, A. (2014). Natural Hazard Zonation of Bihar (India) Using Geoinformatics - A Schematic Approach, Springer International Publishing, New York. 
Kahn, M. (2005). The death toll from natural disasters: The role of income, geography and institutions. Review of Economics and Statistics, 87, 271-284

Levchenko, A. (2004). Institutional quality and international trade. IMF Working Paper, Washington D.C.

Lindell, M. K., \& Prater, C. S. (2003). Assessing community impacts of natural disasters. Natural Hazards Review, 4(4), 176-185.

Méon, P. G., \& Sekkat, K. (2008). Institutional quality and trade: Which institutions? which trade? Economic Inquiry, 46(2), 227-240.

Mechler, R. (2009). Disasters and economic welfare: Can national savings help explain post-disaster changes in consumption? World Bank Policy Research Working Paper 4988. World Bank, Washington, DC.

Munich Re. (2006). Topics geo-annual review: Natural catastrophes 2005. Munich Re Group, German.

Mohan, P., \& Strobl, E. (2012), The economic impact of hurricanes on Caribbean agricultural exports. Working paper, Available

Online:http://www.etsg.org/ETSG2012/Programme/Papers/98.pdf

North, D. W. (1990). Institutions, institutional change and economic performance, Cambridge University Press: Cambridge, UK.

Noy, I. (2009). The macroeconomic consequences of disasters. Journal of Development Economics, 88(2): 221-231.

Nunn, N. (2007). Relationship-specificity, incomplete contracts, and the pattern of trade. The Quarterly Journal of Economics, 122 (2): 569-600.

Raddatz, C. (2007). Are external shocks responsible for the instability of output in low-income countries? Journal of Development Economics, 84 (1): 155-187.

Raddatz, C. (2009). The wrath of god: Macroeconomic costs of natural disasters. Policy Research Working Paper Series 5039, World Bank, Washington D.C.

Ramírez, M. T., \& Loboguerrero, A. M. (2002) Spatial dependence and economic growth: Evidence from a panel of countries. Borradores de Economia Working Paper No. 206. 
Rappaport, E. (2001). Hurricane flooding: A deadly danger. Office of Hydrologic Development, Available Online:

http://www.nws.noaa.gov/oh/hurricane/inland_flooding.html

Rashky, P. (2008). Institutions and the losses from natural disasters. Natural Hazards and Earth System Sciences, 8, 627634.

Rasmussen, T. N. (2004). Macroeconomic implications of natural disasters in the Caribbean. IMF Working Paper No. WP/04/224, IMF, Washington D.C.

Skidmore, M., \& Toya, H. (2002). Do natural disasters promote long-run growth? Economic Inquiry, 40(4): 664-687.

Toya, H., \& Skidmore, M. (2007). Economic development and the impacts of natural disasters. Economics Letters, 94, 20-25 


\section{Tables}

Table 3.1: List of Countries and the Number of Events

\begin{tabular}{|c|c|c|c|c|c|}
\hline Country Name & Region & $\begin{array}{c}\text { \# of All } \\
\text { Disasters }\end{array}$ & $\begin{array}{c}\text { \# of } \\
\text { Severe } \\
\text { Disasters }\end{array}$ & $\begin{array}{c}\text { \# of } \\
\text { Severe } \\
\text { Storms }\end{array}$ & $\begin{array}{c}\text { \# of Named } \\
\text { Hurricanes }\end{array}$ \\
\hline & & (1) & (2) & (3) & (4) \\
\hline Antigua and Barbuda & Caribbean & 5 & 5 & 5 & 1 \\
\hline Bahamas & Caribbean & 10 & 9 & 10 & 25 \\
\hline Barbados & Caribbean & 5 & 3 & 2 & 0 \\
\hline Belize & Central America & 10 & 7 & 6 & 13 \\
\hline Costa Rica & Central America & 31 & 30 & 5 & 3 \\
\hline Cuba & Caribbean & 31 & 28 & 16 & 29 \\
\hline Dominica & Caribbean & 5 & 1 & 1 & 0 \\
\hline Dominican Rep & Caribbean & 36 & 34 & 16 & 13 \\
\hline El Salvador & Central America & 36 & 35 & 9 & 1 \\
\hline Grenada & Caribbean & 4 & 2 & 2 & 1 \\
\hline Guatemala & Central America & 50 & 47 & 8 & 6 \\
\hline Haiti & Caribbean & 57 & 56 & 23 & 10 \\
\hline Honduras & Central America & 41 & 40 & 9 & 16 \\
\hline Jamaica & Caribbean & 18 & 15 & 13 & 11 \\
\hline Mexico & Caribbean & 122 & 122 & 48 & 29 \\
\hline Nicaragua & Central America & 42 & 42 & 11 & 9 \\
\hline Panama & Central America & 28 & 22 & 1 & 1 \\
\hline St Kitts and Nevis & Caribbean & 2 & 2 & 2 & 1 \\
\hline St Lucia & Caribbean & 8 & 5 & 2 & 0 \\
\hline $\begin{array}{l}\text { St Vincent and The } \\
\text { Grenadines }\end{array}$ & Caribbean & 6 & 2 & 2 & 0 \\
\hline Trinidad and Tobago & Caribbean & 7 & 4 & 0 & 0 \\
\hline Total & & 554 & 511 & 191 & 169 \\
\hline
\end{tabular}


Table 3.2: Summary of Used Variables

\begin{tabular}{c|l|l}
\hline Variables & Definition & Source \\
\hline \hline Ln( $\left.\mathrm{Ag}^{\mathrm{i}}\right)$ & Natural logarithm of agriculture import base quantity & UNFAO \\
$\mathrm{Ln}\left(\mathrm{Ag}^{\mathrm{e}}\right)$ & Natural logarithm of agriculture export base quantity & UNFAO \\
$\mathrm{D}_{\mathrm{it}}$ & Dummy variable for natural disasters (severe) & EM-DAT \\
$\mathrm{S}_{\mathrm{it}}$ & Dummy variable for storms (severe) & EM-DAT \\
$\mathrm{Q}$ & Six indicators measuring the quality of institution & WGI \\
GDP & GDP per capita (current US\$) & WB (2005) \\
INV & Gross capital formation (\% of GDP) & WB (2005) \\
FDI & Foreign direct investment, net inflows (BoP, current US\$) & WB (2005) \\
GOV & Government final consumption expenditure (\% of GDP) & WB (2005) \\
POP & Population, total & WB (2005) \\
LAND & Land area (sq. km) & WB (2005) \\
OPEN & Merchandise trade $(\%$ of GDP) & WB (2005) \\
\hline
\end{tabular}


Table 3.3: Correlation between Six Institutional Indicators

\begin{tabular}{c|cccccc} 
& Q1 & Q1 & Q3 & Q4 & Q5 & Q6 \\
\hline Q1 & 1.000 & & & & & \\
Q2 & 0.753 & 1.000 & & & & \\
Q3 & 0.826 & 0.884 & 1.000 & & & \\
Q4 & 0.610 & 0.873 & 0.900 & 1.000 & & \\
Q5 & 0.671 & 0.773 & 0.885 & 0.876 & 1.000 & \\
Q6 & 0.876 & 0.827 & 0.797 & 0.621 & 0.592 & 1.000 \\
\hline \hline
\end{tabular}

Q1 = Voice and Accountability; Q2 = Government Effectiveness; Q3 = Rule of Law; Q4 = Control of Corruption; Q5 = Political Stability; Q6 = Regulatory Quality 
Table 3.4: Explanatory Power of Six Institutional Indicators

\begin{tabular}{|c|c|c|c|c|c|c|}
\hline $\begin{array}{l}\text { Dependent } \\
\text { Variable: LnAge }\end{array}$ & (1) & (2) & (3) & (4) & $(5)$ & (6) \\
\hline " & $-0.116^{*}$ & & & & & \\
\hline Accountability & $(0.064)$ & & & & & \\
\hline $\mathrm{D}_{\mathrm{t}-2} *$ Voice and & $-0.122 *$ & & & & & \\
\hline Accountability & $(0.069)$ & & & & & \\
\hline $\mathrm{D}_{\mathrm{t}-3} *$ Voice and & -0.035 & & & & & \\
\hline Accountability & $(0.068)$ & & & & & \\
\hline $\mathrm{D}_{\mathrm{t}-1} *$ Government & & -0.1094 & & & & \\
\hline Effectiveness & & $(0.070)$ & & & & \\
\hline $\mathrm{D}_{\mathrm{t}-2}{ }^{*}$ Government & & $-0.164 * *$ & & & & \\
\hline Effectiveness & & $(0.076)$ & & & & \\
\hline $\mathrm{D}_{\mathrm{t}-3} *$ Government & & -0.0556 & & & & \\
\hline \multirow[t]{2}{*}{ Effectiveness } & & $(0.072)$ & & & & \\
\hline & & & -0.0692 & & & \\
\hline \multirow[t]{2}{*}{$\mathrm{D}_{\mathrm{t}-1} *$ Rule of Law } & & & $(0.056)$ & & & \\
\hline & & & $-0.154 * *$ & & & \\
\hline \multirow{2}{*}{$\mathrm{D}_{\mathrm{t}-2} *$ Rule of Law } & & & $(0.060)$ & & & \\
\hline & & & $-0.099 *$ & & & \\
\hline $\mathrm{D}_{\mathrm{t}-3} *$ Rule of Law & & & $(0.057)$ & & & \\
\hline $\mathrm{D}_{\mathrm{t}-1} *$ Control of & & & & $-0.095^{*}$ & & \\
\hline Corruption & & & & $(0.058)$ & & \\
\hline $\mathrm{D}_{\mathrm{t}-2} *$ Control of & & & & $-0.182 * * *$ & & \\
\hline Corruption & & & & $(0.062)$ & & \\
\hline $\mathrm{D}_{\mathrm{t}-3} *$ Control of & & & & $-0.109 *$ & & \\
\hline Corruption & & & & $(0.060)$ & & \\
\hline $\mathrm{D}_{\mathrm{t}-1} *$ Political & & & & & -0.0731 & \\
\hline Stability & & & & & $(0.075)$ & \\
\hline $\mathrm{D}_{\mathrm{t}-2} *$ Political & & & & & $-0.168 * *$ & \\
\hline Stability & & & & & $(0.080)$ & \\
\hline $\mathrm{D}_{\mathrm{t}-3} *$ Political & & & & & -0.0632 & \\
\hline Stability & & & & & $(0.075)$ & \\
\hline $\mathrm{D}_{\mathrm{t}-1} *$ Regulatory & & & & & & -0.0745 \\
\hline Quality & & & & & & $(0.076)$ \\
\hline $\mathrm{D}_{\mathrm{t}-2} *$ Regulatory & & & & & & -0.1267 \\
\hline Quality & & & & & & $(0.082)$ \\
\hline $\mathrm{D}_{\mathrm{t}-3} *$ Regulatory & & & & & & -0.0148 \\
\hline Quality & & & & & & $(0.084)$ \\
\hline Obs & 311 & 305 & 311 & 305 & 304 & 305 \\
\hline $\mathrm{R}^{2}$ & 0.5273 & 0.5192 & 0.5271 & 0.522 & 0.5044 & 0.5186 \\
\hline
\end{tabular}


Table 3.5: The Impact of Disasters on Agricultural Imports

\begin{tabular}{|c|c|c|c|c|c|c|}
\hline \multirow{3}{*}{$\begin{array}{l}\text { Dep. Var. } \\
\operatorname{Ln}\left(\mathrm{Ag}^{\mathrm{i}}\right)\end{array}$} & \multirow{2}{*}{\multicolumn{2}{|c|}{ Panel Regression }} & \multicolumn{4}{|c|}{ Spatial Panel Regression } \\
\hline & & & Contiguity & NN1 & $\mathrm{NN} 2$ & NN4 \\
\hline & (1) & (2) & (3) & (4) & (5) & (6) \\
\hline \multirow[t]{2}{*}{$\mathrm{D}_{\mathrm{t}}$} & $0.059^{* *}$ & $0.063^{* *}$ & 0.043 & $0.041^{*}$ & $0.036^{*}$ & 0.030 \\
\hline & $(0.03)$ & $(0.03)$ & $(0.03)$ & $(0.02)$ & $(0.02)$ & $(0.02)$ \\
\hline \multirow[t]{2}{*}{$D_{t-1}$} & & $0.064^{* *}$ & $0.084^{* * * *}$ & $0.061^{* * *}$ & $0.057^{* * *}$ & $0.061^{* * * *}$ \\
\hline & & $(0.03)$ & $(0.03)$ & $(0.02)$ & $(0.02)$ & $(0.02)$ \\
\hline \multirow[t]{2}{*}{$\mathrm{D}_{\mathrm{t}-2}$} & & 0.037 & $0.051^{*}$ & 0.024 & $0.038^{*}$ & $0.041^{* *}$ \\
\hline & & $(0.03)$ & $(0.03)$ & $(0.02)$ & $(0.02)$ & $(0.02)$ \\
\hline \multirow[t]{2}{*}{$D_{t-3}$} & & 0.029 & $0.062^{* *}$ & 0.024 & 0.019 & 0.017 \\
\hline & & $(0.03)$ & $(0.03)$ & $(0.02)$ & $(0.02)$ & $(0.02)$ \\
\hline \multirow[t]{2}{*}{ LnGDP } & $0.571^{* * *}$ & $0.549^{* * *}$ & $0.089^{* * * *}$ & $0.077^{* * *}$ & $0.039^{* *}$ & $0.038^{* *}$ \\
\hline & $(0.06)$ & $(0.06)$ & $(0.02)$ & $(0.02)$ & $(0.02)$ & $(0.02)$ \\
\hline \multirow[t]{2}{*}{ LnFDI } & -0.001 & -0.001 & $0.018^{* * * *}$ & $0.010^{* * *}$ & $0.010^{* * *}$ & $0.008^{* *}$ \\
\hline & $(0.02)$ & $(0.02)$ & $(0.00)$ & $(0.00)$ & $(0.00)$ & $(0.00)$ \\
\hline \multirow[t]{2}{*}{ LnINV } & $0.117^{*}$ & $0.122^{*}$ & 0.027 & -0.004 & -0.013 & $-0.032^{*}$ \\
\hline & $(0.06)$ & $(0.06)$ & $(0.02)$ & $(0.02)$ & $(0.02)$ & $(0.02)$ \\
\hline \multirow[t]{2}{*}{ LnGOV } & -0.061 & -0.102 & $-0.114^{* * *}$ & $-0.067^{* * *}$ & $-0.040^{* *}$ & -0.018 \\
\hline & $(0.07)$ & $(0.07)$ & $(0.02)$ & $(0.02)$ & $(0.02)$ & $(0.02)$ \\
\hline \multirow[t]{2}{*}{ LnPOP } & -0.071 & -0.038 & $0.705^{* * *}$ & 0.194 & 0.067 & 0.065 \\
\hline & $(0.25)$ & $(0.25)$ & $(0.18)$ & $(0.16)$ & $(0.14)$ & $(0.14)$ \\
\hline \multirow[t]{2}{*}{ LnLAND } & 12.644 & 8.301 & $-19.791^{*}$ & -7.397 & 2.660 & 5.127 \\
\hline & $(11.86)$ & (11.97) & $(10.66)$ & $(9.18)$ & $(8.35)$ & (8.08) \\
\hline \multirow[t]{2}{*}{ LnOPEN } & 0.059 & 0.025 & $-0.252^{* * * *}$ & $-0.194^{* * *}$ & $-0.132^{* * * *}$ & $-0.109^{* * * *}$ \\
\hline & $(0.06)$ & $(0.06)$ & $(0.05)$ & $(0.04)$ & $(0.04)$ & $(0.04)$ \\
\hline \multirow[t]{2}{*}{ Constant } & -112.163 & -70.915 & $187.622^{*}$ & 72.694 & -22.931 & -47.409 \\
\hline & $(112.46)$ & $(113.51)$ & (101.79) & (87.56) & $(79.61)$ & (77.10) \\
\hline \multirow[t]{2}{*}{ Rho } & & & $0.263^{* * *}$ & $0.610^{* * * *}$ & $0.374^{* * *}$ & $0.207^{* * *}$ \\
\hline & & & $(0.03)$ & $(0.04)$ & $(0.02)$ & $(0.01)$ \\
\hline Observations & 309 & 309 & 336 & 336 & 336 & 336 \\
\hline Groups & 21 & 21 & 21 & 21 & 21 & 21 \\
\hline R-Square & 0.553 & 0.558 & 0.987 & 0.991 & 0.992 & 0.993 \\
\hline
\end{tabular}


Table 3.6: The Impact of Disasters on Agricultural Exports

\begin{tabular}{|c|c|c|c|c|c|c|}
\hline \multirow{3}{*}{$\begin{array}{l}\text { Dep. Var. } \\
\operatorname{Ln}\left(\mathrm{Ag}^{\mathrm{e}}\right)\end{array}$} & \multirow{2}{*}{\multicolumn{2}{|c|}{ Panel Regression }} & \multicolumn{4}{|c|}{ Spatial Panel Regression } \\
\hline & & & Contiguity & NN1 & $\mathrm{NN} 2$ & NN4 \\
\hline & (1) & (2) & (3) & (4) & (5) & (6) \\
\hline \multirow[t]{2}{*}{$\mathrm{D}_{\mathrm{t}}$} & -0.009 & -0.012 & -0.035 & -0.033 & -0.037 & -0.047 \\
\hline & $(0.05$ & $(0.05)$ & $(0.04)$ & $(0.05)$ & $(0.04)$ & $(0.04)$ \\
\hline \multirow[t]{2}{*}{$\mathrm{D}_{\mathrm{t}-1}$} & & $-0.152^{* * * *}$ & $-0.124^{* * *}$ & $-0.122^{* * *}$ & $-0.112^{* * *}$ & $-0.124^{* * *}$ \\
\hline & & $(0.04)$ & $(0.04)$ & $(0.04)$ & $(0.04)$ & $(0.04)$ \\
\hline \multirow[t]{2}{*}{$D_{t-2}$} & & $-0.175^{* * * *}$ & $-0.140^{* * * *}$ & $-0.143^{* * * *}$ & $-0.136^{* * * *}$ & $-0.137^{* * * *}$ \\
\hline & & $(0.05)$ & $(0.04)$ & $(0.04)$ & $(0.04)$ & $(0.04)$ \\
\hline \multirow[t]{2}{*}{$D_{t-3}$} & & $-0.172^{* * * *}$ & $-0.106^{* *}$ & $-0.112^{* * * *}$ & $-0.110^{* *}$ & $-0.122^{* * * *}$ \\
\hline & & $(0.04)$ & $(0.04)$ & $(0.04)$ & $(0.04)$ & $(0.04)$ \\
\hline \multirow[t]{2}{*}{ LnGDP } & $0.431^{* * *}$ & $0.526^{* * *}$ & $0.066^{*}$ & 0.051 & 0.044 & 0.025 \\
\hline & $(0.11)$ & $(0.10)$ & $(0.03)$ & $(0.03)$ & $(0.03)$ & $(0.03)$ \\
\hline \multirow{2}{*}{ LnFDI } & 0.035 & 0.035 & 0.007 & 0.007 & 0.007 & 0.004 \\
\hline & $(0.03)$ & $(0.03)$ & $(0.01)$ & $(0.01)$ & $(0.01)$ & $(0.01)$ \\
\hline \multirow[t]{2}{*}{ LnINV } & 0.169 & 0.136 & $0.092^{* *}$ & $0.071^{*}$ & $0.071^{* *}$ & $0.089^{* * *}$ \\
\hline & $(0.11)$ & $(0.11)$ & $(0.04)$ & $(0.04)$ & $(0.04)$ & $(0.03)$ \\
\hline \multirow[t]{2}{*}{ LnGOV } & $-0.229^{*}$ & -0.062 & $-0.145^{* * *}$ & $-0.109^{* * * *}$ & $-0.099^{* *}$ & $-0.090^{* *}$ \\
\hline & $(0.13)$ & $(0.12)$ & $(0.04)$ & $(0.04)$ & $(0.04)$ & $(0.04)$ \\
\hline \multirow[t]{2}{*}{ LnPOP } & 0.100 & -0.085 & $1.352^{* * * *}$ & $1.400^{* * * *}$ & $1.247^{* * * *}$ & $1.047^{* * *}$ \\
\hline & $(0.43)$ & $(0.41)$ & $(0.27)$ & $(0.27)$ & $(0.28)$ & $(0.26)$ \\
\hline \multirow[t]{2}{*}{ LnLAND } & -29.978 & -9.590 & $-50.963^{\text {*** }}$ & $-46.202^{* * * *}$ & $-57.432^{* * *}$ & $-58.370^{* * *}$ \\
\hline & (20.74) & (20.01) & (17.11) & $(17.30)$ & (17.19) & (16.38) \\
\hline \multirow[t]{2}{*}{ LnOPEN } & -0.056 & 0.085 & $-0.211^{* * *}$ & $-0.177^{* * *}$ & $-0.140^{*}$ & -0.081 \\
\hline & $(0.10)$ & $(0.10)$ & $(0.08)$ & $(0.08)$ & $(0.08)$ & $(0.08)$ \\
\hline \multirow[t]{2}{*}{ Constant } & 291.968 & 98.923 & $475.359^{* * *}$ & $429.987^{\text {**** }}$ & $537.834^{* * * *}$ & $546.694^{* * *}$ \\
\hline & (196.68) & (189.70) & (163.38) & $(165.20)$ & $(164.20)$ & (156.46) \\
\hline \multirow[t]{2}{*}{ Rho } & & & $0.234^{* * * *}$ & $0.197^{* * * *}$ & $0.153^{* * * *}$ & $0.138^{* * * *}$ \\
\hline & & & $(0.05)$ & $(0.06)$ & $(0.04)$ & $(0.02)$ \\
\hline Observations & 309 & 309 & 336 & 336 & 336 & 336 \\
\hline Groups & 21 & 21 & 21 & 21 & 21 & 21 \\
\hline R-Square & 0.553 & 0.552 & 0.985 & 0.985 & 0.985 & 0.986 \\
\hline
\end{tabular}


Table 3.7: The Role of the Institution

\begin{tabular}{|c|c|c|c|c|c|c|}
\hline \multirow[t]{2}{*}{ Dep. Var. } & \multicolumn{3}{|c|}{$\operatorname{Ln}\left(\mathrm{Ag}^{\mathrm{i}}\right)$} & \multicolumn{3}{|c|}{$\operatorname{Ln}\left(\mathrm{Ag}^{\mathrm{e}}\right)$} \\
\hline & (1) Panel & (2) NN1 & (3) NN2 & (4) Panel & (5) NN1 & (6) NN2 \\
\hline \multirow[t]{2}{*}{$\mathrm{D}_{\mathrm{t}}$} & $0.068^{* *}$ & $0.043^{*}$ & $0.038^{*}$ & -0.016 & -0.041 & -0.043 \\
\hline & $(0.03)$ & $(0.02)$ & $(0.02)$ & $(0.05)$ & $(0.05)$ & $(0.05)$ \\
\hline \multirow[t]{2}{*}{$D_{t-1}$} & $0.054^{* *}$ & $0.053^{* *}$ & $0.050^{* *}$ & $-0.157^{* * *}$ & $-0.123^{* * *}$ & $-0.114^{* * *}$ \\
\hline & $(0.03)$ & $(0.02)$ & $(0.02)$ & $(0.04)$ & $(0.04)$ & $(0.04)$ \\
\hline \multirow[t]{2}{*}{$D_{t-2}$} & 0.028 & 0.023 & $0.038^{*}$ & $-0.194^{* * *}$ & $-0.142^{* * *}$ & $-0.136^{* * *}$ \\
\hline & $(0.03)$ & $(0.02)$ & $(0.02)$ & $(0.05)$ & $(0.04)$ & $(0.04)$ \\
\hline \multirow[t]{2}{*}{$D_{t-3}$} & 0.030 & 0.022 & 0.020 & $-0.169^{* * *}$ & $-0.107^{* *}$ & $-0.106^{* *}$ \\
\hline & $(0.03)$ & $(0.02)$ & $(0.02)$ & $(0.04)$ & $(0.04)$ & $(0.04)$ \\
\hline \multirow[t]{2}{*}{$\mathrm{D}_{\mathrm{t}}^{*} \mathrm{Q}$} & -0.006 & 0.025 & 0.010 & -0.052 & -0.022 & -0.025 \\
\hline & $(0.03)$ & $(0.03)$ & $(0.03)$ & $(0.05)$ & $(0.05)$ & $(0.05)$ \\
\hline \multirow[t]{2}{*}{$D_{t-1} * Q$} & 0.034 & 0.042 & 0.031 & $-0.123^{* *}$ & -0.074 & -0.068 \\
\hline & $(0.03)$ & $(0.03)$ & $(0.03)$ & $(0.05)$ & $(0.05)$ & $(0.05)$ \\
\hline \multirow{2}{*}{$\mathrm{D}_{\mathrm{t}-2} * \mathrm{Q}$} & 0.005 & 0.035 & 0.012 & $-0.169^{* * * *}$ & $-0.150^{* * *}$ & $-0.145^{* * *}$ \\
\hline & $(0.03)$ & $(0.03)$ & $(0.03)$ & $(0.06)$ & $(0.06)$ & $(0.06)$ \\
\hline \multirow[t]{2}{*}{$\mathrm{D}_{\mathrm{t}-3} * \mathrm{Q}$} & -0.005 & -0.028 & -0.032 & $-0.113^{* *}$ & $-0.108^{* *}$ & $-0.113^{* *}$ \\
\hline & $(0.03)$ & $(0.03)$ & $(0.03)$ & $(0.05)$ & $(0.05)$ & $(0.05)$ \\
\hline \multirow[t]{2}{*}{ Q } & $0.115^{* *}$ & -0.066 & -0.025 & $0.142^{*}$ & 0.026 & -0.002 \\
\hline & $(0.05)$ & $(0.04)$ & $(0.04)$ & $(0.08)$ & $(0.08)$ & $(0.07)$ \\
\hline \multirow[t]{2}{*}{ LnGDP } & $0.517^{* * *}$ & $0.076^{* * *}$ & $0.038^{* *}$ & $0.571^{* * *}$ & $0.065^{*}$ & $0.059^{*}$ \\
\hline & $(0.06)$ & $(0.02)$ & $(0.02)$ & $(0.10)$ & $(0.03)$ & $(0.03)$ \\
\hline \multirow[t]{2}{*}{ LnFDI } & 0.009 & $0.011^{* * *}$ & $0.010^{* * *}$ & 0.031 & 0.006 & 0.005 \\
\hline & $(0.02)$ & $(0.00)$ & $(0.00)$ & $(0.03)$ & $(0.01)$ & $(0.01)$ \\
\hline \multirow[t]{2}{*}{ LnINV } & 0.092 & 0.002 & -0.011 & 0.118 & 0.059 & $0.062^{*}$ \\
\hline & $(0.07)$ & $(0.02)$ & $(0.02)$ & $(0.11)$ & $(0.04)$ & $(0.04)$ \\
\hline \multirow[t]{2}{*}{ LnGOV } & $-0.132^{*}$ & $-0.069^{* * *}$ & $-0.039^{* *}$ & -0.127 & $-0.105^{* * *}$ & $-0.096^{* *}$ \\
\hline & $(0.08)$ & $(0.02)$ & $(0.02)$ & $(0.13)$ & $(0.04)$ & $(0.04)$ \\
\hline \multirow[t]{2}{*}{ LnPOP } & 0.053 & 0.214 & 0.087 & -0.098 & $1.367^{* * *}$ & $1.214^{* * *}$ \\
\hline & $(0.25)$ & $(0.16)$ & $(0.15)$ & $(0.41)$ & $(0.27)$ & $(0.27)$ \\
\hline \multirow[t]{2}{*}{ LnLAND } & 4.665 & -7.226 & 3.093 & 0.560 & $-36.029^{* *}$ & $-46.833^{* * *}$ \\
\hline & (11.90) & $(9.31)$ & $(8.52)$ & (19.64) & $(17.31)$ & $(17.15)$ \\
\hline \multirow[t]{2}{*}{ LnOPEN } & 0.015 & $-0.195^{* * *}$ & $-0.134^{* * *}$ & 0.023 & $-0.215^{* * *}$ & $-0.181^{* *}$ \\
\hline & $(0.06)$ & $(0.04)$ & $(0.04)$ & $(0.10)$ & $(0.08)$ & $(0.08)$ \\
\hline \multirow[t]{2}{*}{ Rho } & & $0.624^{* * *}$ & $0.375^{* * *}$ & & $0.196^{* * *}$ & $0.157^{* * *}$ \\
\hline & & $(0.05)$ & $(0.02)$ & & $(0.06)$ & $(0.04)$ \\
\hline Obs & 303 & 336 & 336 & 303 & 336 & 336 \\
\hline R-Square & 0.555 & 0.991 & 0.992 & 0.559 & 0.985 & 0.985 \\
\hline
\end{tabular}


Table 3.8: Regression Results on the Impact of Storms

Data Source: EM-DAT

\begin{tabular}{|c|c|c|c|c|c|c|c|c|}
\hline & \multicolumn{4}{|c|}{ Dummy Effects } & \multicolumn{4}{|c|}{ Frequency Effects } \\
\hline & \multicolumn{8}{|c|}{ Dependent Variable } \\
\hline & \multicolumn{2}{|c|}{$\ln \mathrm{Ag}^{\mathrm{i}}$} & \multicolumn{2}{|c|}{$\ln \mathrm{Ag}^{\mathrm{e}}$} & \multicolumn{2}{|c|}{$\ln \mathrm{Ag}^{\mathrm{i}}$} & \multicolumn{2}{|c|}{$\ln \mathrm{Ag}^{\mathrm{e}}$} \\
\hline & $(1)$ & $(2)$ & (3) & $(4)$ & $(5)$ & $(6)$ & $(7)$ & (8) \\
\hline $\mathrm{S}_{\mathrm{t}}$ & $\begin{array}{l}0.0755 * * * \\
(0.025)\end{array}$ & $\begin{array}{l}0.0776^{* * *} \\
(0.024)\end{array}$ & $\begin{array}{l}0.0160 \\
(0.050)\end{array}$ & $\begin{array}{l}0.0062 \\
(0.045)\end{array}$ & $\begin{array}{l}0.0315^{* *} \\
(0.013)\end{array}$ & $\begin{array}{l}0.0383 * * * \\
(0.014)\end{array}$ & $\begin{array}{l}-0.0105 \\
(0.026)\end{array}$ & $\begin{array}{l}-0.0246 \\
(0.026)\end{array}$ \\
\hline $\mathrm{S}_{\mathrm{t}-1}$ & $\begin{array}{l}0.0816 * * * \\
(0.024)\end{array}$ & $\begin{array}{l}0.0724 * * * \\
(0.024)\end{array}$ & $\begin{array}{l}-0.0815^{*} \\
(0.048)\end{array}$ & $\begin{array}{l}-0.0810^{*} \\
(0.044)\end{array}$ & $\begin{array}{l}0.0409 * * * \\
(0.013)\end{array}$ & $\begin{array}{l}0.0433 * * * \\
(0.014)\end{array}$ & $\begin{array}{l}-0.0422^{*} \\
(0.026)\end{array}$ & $\begin{array}{l}-0.0721^{* * * *} \\
(0.025)\end{array}$ \\
\hline $\mathrm{S}_{\mathrm{t}-2}$ & $\begin{array}{l}0.0312 \\
(0.025)\end{array}$ & $\begin{array}{l}0.0218 \\
(0.025)\end{array}$ & $\begin{array}{l}-0.1393^{* * * *} \\
(0.050)\end{array}$ & $\begin{array}{l}-0.1423^{* * *} \\
(0.046)\end{array}$ & $\begin{array}{l}0.0260 * \\
(0.014)\end{array}$ & $\begin{array}{l}0.0259 * \\
(0.015)\end{array}$ & $\begin{array}{l}-0.0728 * * \\
(0.028)\end{array}$ & $\begin{array}{l}-0.1014^{* * * *} \\
(0.027)\end{array}$ \\
\hline $\mathrm{S}_{\mathrm{t}-3}$ & $\begin{array}{l}0.0230 \\
(0.025)\end{array}$ & $\begin{array}{l}0.0197 \\
(0.025)\end{array}$ & $\begin{array}{l}-0.1109^{* * *} \\
(0.050)\end{array}$ & $\begin{array}{l}-0.1007^{* * *} \\
(0.046)\end{array}$ & $\begin{array}{l}0.0363 * \\
(0.014)\end{array}$ & $\begin{array}{l}0.0339 * \\
(0.015)\end{array}$ & $\begin{array}{l}-0.040 \\
(0.028)\end{array}$ & $\begin{array}{l}-0.0639 * * \\
(0.027)\end{array}$ \\
\hline $\mathrm{St}_{\mathrm{t}}^{*} \mathrm{Q}$ & & $\begin{array}{l}-0.0043 \\
(0.029)\end{array}$ & & $\begin{array}{l}-0.0667 \\
(0.054)\end{array}$ & & $\begin{array}{l}0.0155 \\
(0.017)\end{array}$ & & $\begin{array}{l}-0.0162 \\
(0.032)\end{array}$ \\
\hline $\mathrm{S}_{\mathrm{t}-1} * \mathrm{Q}$ & & $\begin{array}{l}0.0046 \\
(0.030)\end{array}$ & & $\begin{array}{l}-0.1357^{* *} \\
(0.055)\end{array}$ & & $\begin{array}{l}0.0234 \\
(0.017)\end{array}$ & & $\begin{array}{l}-0.0667^{* *} \\
(0.031)\end{array}$ \\
\hline $\mathrm{S}_{\mathrm{t}-2} * \mathrm{Q}$ & & $\begin{array}{l}-0.0022 \\
(0.033)\end{array}$ & & $\begin{array}{l}-0.2036^{* * * *} \\
(0.060)\end{array}$ & & $\begin{array}{l}0.0033 \\
(0.020)\end{array}$ & & $\begin{array}{l}-0.1417^{* * * *} \\
(0.036)\end{array}$ \\
\hline $\mathrm{S}_{\mathrm{t}-3} * \mathrm{Q}$ & & $\begin{array}{l}-0.0179 \\
(0.031)\end{array}$ & & $\begin{array}{l}-0.1539 * * * \\
(0.057)\end{array}$ & & $\begin{array}{l}-0.0137 \\
(0.019)\end{array}$ & & $\begin{array}{l}-0.1084^{* * * *} \\
(0.036)\end{array}$ \\
\hline Obs & 320 & 308 & 317 & 305 & 320 & 308 & 317 & 305 \\
\hline $\mathrm{R}^{2}$ & 0.5115 & 0.5183 & 0.5135 & 0.5143 & 0.5088 & 0.5146 & 0.5142 & 0.5188 \\
\hline
\end{tabular}


Table 3.9: Regression Results on the Impact of Hurricanes

Data Source: NHC

\begin{tabular}{|c|c|c|c|c|c|c|c|c|}
\hline & \multicolumn{4}{|c|}{ Dummy Effects } & \multicolumn{4}{|c|}{ Frequency Effects } \\
\hline & \multicolumn{8}{|c|}{ Dependent Variable } \\
\hline & \multicolumn{2}{|c|}{$\ln \mathrm{Ag}^{\mathrm{i}}$} & \multicolumn{2}{|c|}{$\ln \mathrm{Ag}^{\mathrm{e}}$} & \multicolumn{2}{|c|}{$\ln \mathrm{Ag}^{\mathrm{i}}$} & \multicolumn{2}{|c|}{$\ln \mathrm{Ag}^{\mathrm{e}}$} \\
\hline & (1) & (2) & (3) & (4) & (5) & (6) & (7) & (8) \\
\hline $\mathrm{H}_{\mathrm{t}}$ & $\begin{array}{l}0.0671^{* *} \\
(0.029)\end{array}$ & $\begin{array}{l}0.0772^{* * * *} \\
(0.030)\end{array}$ & $\begin{array}{l}-0.0103 \\
(0.054)\end{array}$ & $\begin{array}{l}-0.0393 \\
(0.054)\end{array}$ & $\begin{array}{l}0.0351 * * * \\
(0.011)\end{array}$ & $\begin{array}{l}0.0386^{* * *} \\
(0.011)\end{array}$ & $\begin{array}{l}-0.0164 \\
(0.022)\end{array}$ & $\begin{array}{l}-0.0156 \\
(0.021)\end{array}$ \\
\hline $\mathrm{H}_{\mathrm{t}-1}$ & $\begin{array}{l}0.0518 * \\
(0.028)\end{array}$ & $\begin{array}{l}0.0628 * * \\
(0.029)\end{array}$ & $\begin{array}{l}-0.0609^{* *} \\
(0.053)\end{array}$ & $\begin{array}{l}-0.0694^{* * *} \\
(0.052)\end{array}$ & $\begin{array}{l}0.0325^{* * *} \\
(0.011)\end{array}$ & $\begin{array}{l}0.0362 * * * \\
(0.011)\end{array}$ & $\begin{array}{l}-0.0448 * * \\
(0.022)\end{array}$ & $\begin{array}{l}-0.0405^{* * *} \\
(0.021)\end{array}$ \\
\hline $\mathrm{H}_{\mathrm{t}-2}$ & $\begin{array}{l}0.0662 * * \\
(0.027)\end{array}$ & $\begin{array}{l}0.0608 * * \\
(0.028)\end{array}$ & $\begin{array}{l}-0.1104^{* * * *} \\
(0.052)\end{array}$ & $\begin{array}{l}-0.1368^{* * * *} \\
(0.051)\end{array}$ & $\begin{array}{l}0.0413^{* * *} \\
(0.012)\end{array}$ & $\begin{array}{l}0.0420 * * * \\
(0.0120\end{array}$ & $\begin{array}{l}-0.0864^{* * * *} \\
(0.023)\end{array}$ & $\begin{array}{l}-0.0828 * * * \\
(0.022)\end{array}$ \\
\hline $\mathrm{H}_{\mathrm{t}-3}$ & $\begin{array}{l}0.0816^{* * * *} \\
(0.027)\end{array}$ & $\begin{array}{l}0.0714^{* * * *} \\
(0.027)\end{array}$ & $\begin{array}{l}-0.0253^{*} \\
(0.051)\end{array}$ & $\begin{array}{l}-0.0385^{*} \\
(0.050)\end{array}$ & $\begin{array}{l}0.0550 * * * \\
(0.012)\end{array}$ & $\begin{array}{l}0.0530 * * * \\
(0.012)\end{array}$ & $\begin{array}{l}-0.0013 \\
(0.023)\end{array}$ & $\begin{array}{l}-0.0023 \\
(0.022)\end{array}$ \\
\hline $\mathrm{H}_{\mathrm{t}}^{*} \mathrm{Q}$ & & $\begin{array}{l}-0.0030 \\
(0.038)\end{array}$ & & $\begin{array}{l}-0.0359^{*} \\
(0.070)\end{array}$ & & $\begin{array}{l}0.0084 \\
(0.014)\end{array}$ & & $\begin{array}{l}-0.0323 \\
(0.027)\end{array}$ \\
\hline $\mathrm{H}_{\mathrm{t}-1} * \mathrm{Q}$ & & $\begin{array}{l}0.0231 \\
(0.037)\end{array}$ & & $\begin{array}{l}-0.2723^{* * * *} \\
(0.067)\end{array}$ & & $\begin{array}{l}0.0158 \\
(0.014)\end{array}$ & & $\begin{array}{l}-0.0995^{* * * *} \\
(0.026)\end{array}$ \\
\hline $\mathrm{H}_{\mathrm{t}-2} * \mathrm{Q}$ & & $\begin{array}{l}-0.0253 \\
(0.037)\end{array}$ & & $\begin{array}{l}-0.2218^{* * *} \\
(0.067)\end{array}$ & & $\begin{array}{l}0.0024 \\
(0.0140\end{array}$ & & $\begin{array}{l}-0.0949 * * * \\
(0.027)\end{array}$ \\
\hline $\mathrm{H}_{\mathrm{t}-3} * \mathrm{Q}$ & & $\begin{array}{l}-0.0344 \\
(0.037)\end{array}$ & & $\begin{array}{l}-0.1755^{* * *} \\
(0.067)\end{array}$ & & $\begin{array}{l}-0.0062 \\
(0.015)\end{array}$ & & $\begin{array}{l}-0.0614^{* * *} \\
(0.028)\end{array}$ \\
\hline Obs & 310 & 304 & 310 & 304 & 310 & 304 & 310 & 304 \\
\hline $\mathrm{R}^{2}$ & 0.5168 & 0.5061 & 0.5244 & 0.5129 & 0.5129 & 0.500 & 0.5243 & 0.5161 \\
\hline
\end{tabular}




\section{Figures}

Figure 3.1: Natural Disaster Subgroup Definition and Classification

\begin{tabular}{|c|c|c|}
\hline $\begin{array}{l}\text { Disaster } \\
\text { Subgroup }\end{array}$ & Definition & Disaster Main Types \\
\hline Geophysical & Events originating from solid earth & $\begin{array}{l}\text { Earthquake, Volcano, Mass } \\
\text { Movement (dry) }\end{array}$ \\
\hline Meteorological & $\begin{array}{l}\text { Events caused by short-lived/small to meso scale } \\
\text { atmospheric processes (in the spectrum from } \\
\text { minutes to days) }\end{array}$ & Storm \\
\hline Hydrological & $\begin{array}{l}\text { Events caused by deviations in the normal water } \\
\text { cycle and/or overflow of bodies of water caused } \\
\text { by wind set-up }\end{array}$ & Flood, Mass Movement (wet) \\
\hline Climatological & $\begin{array}{l}\text { Events caused by long-lived/meso to macro scale } \\
\text { processes (in the spectrum from intra-seasonal to } \\
\text { multi-decadal climate variability) }\end{array}$ & $\begin{array}{l}\text { Extreme Temperature, } \\
\text { Drought, Wildfire }\end{array}$ \\
\hline Biological ${ }^{4}$ & $\begin{array}{l}\text { Disaster caused by the exposure of living } \\
\text { organisms to germs and toxic substances }\end{array}$ & $\begin{array}{l}\text { Epidemic, Insect Infestation, } \\
\text { Animal Stampede }\end{array}$ \\
\hline
\end{tabular}

Notes: This figure is adopted from Guha-Sapir and Hoyois (2013). Annual Disaster Statistical Review 2012: The Numbers and Trends. Brussels: CRED. 
Figure 3.2: Number of Disasters and Storms by Country
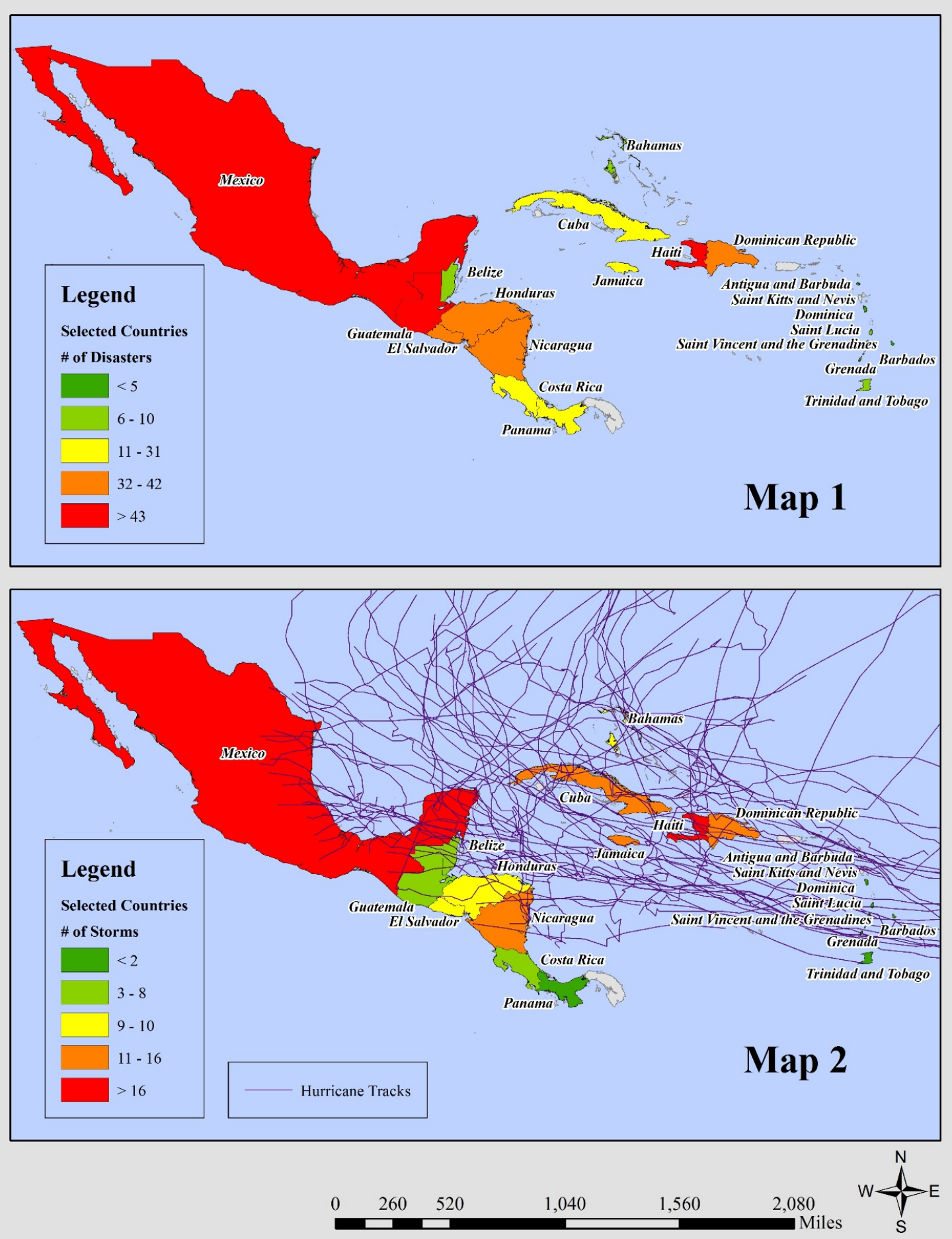
VITA

\section{SISI MENG}

2016

Ph.D. Economics, Candidate

Florida International University

2013

M.A., Economics

Florida International University

Miami, FL

2011

M.S., Economics

University of Illinois at Urbana-Champaign

Urbana, IL

2010

B.S., Economics

Capital University of Economics and Business

Bejing, CHINA

\section{PUBLICATIONS AND PRESENTATIONS}

Meng, S., \& P. Mozumder. (2015). Hurricane Sandy: Damages, Critical Infrastructure Disruptions and Pathways to Recovery, Under Review.

Meng, S., \& P. Mozumder. (2015). Household Preferences for Sea Level Rise Adaptation Plan. Presented at Southern Economic Association, 85th Annual Conference at New Orleans, Louisiana (Nov 21-23, 2015).

Meng, S., \& P. Mozumder. (2015). The Determinants of Property Damage: Evidence from Hurricane Sandy. Presented at Western Economic Association International, 90th Annual Conference at Honolulu, Hawaii (Jun 28- Jul 2, 2015).

Meng, S., \& P. Mozumder. (2015). Institutions and the Impact of Natural Disasters: Evidence from Agricultural Trade Flows in Central America and the Caribbean. Presented at Urban Affair Association, 45thAnnual Conference, Miami, Florida (Apr 8-11, 2015).

Vasquez, W. F., T. J. Murray., S. Meng., \& P. Mozumder. (2016). An Empirical Analysis of Determinants Underlying Perceptions of Impending Hurricanes, Under Review. 